\title{
Decoerência e Dissipação de Sistemas Quânticos: Técnicas e Aplicaçōes
}

Sonia Geraij Mokatzel

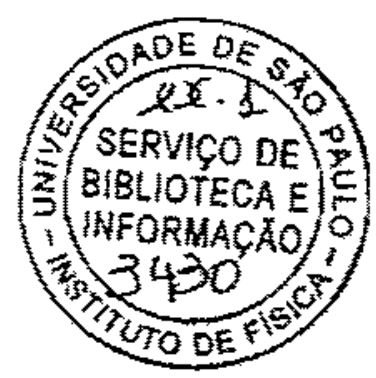

Tese de Doutorado

submetida ao Instituto de Física da Universidade de São Paulo

\author{
M.C. Women
}

ORIENTADORA: Prof ${ }^{a}$ Dra. Maria Carolina Nemes

Comiss

Prof Dra. Maria Carolina Nemes - UFMG

Prof. Dr Autônio Fonando Ribeiro de Toledo Piza - USP

Prof. Dr Carlos Ourvio Escobar - UNICAMP

Prof. Dr Nicin Zagury - UFRJ

Prot. Dr Salomon Sylwan Mizahi-UFSCAR

SÃO PAULO 2000

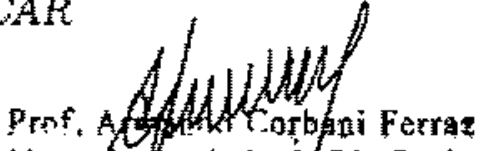
resilucricta fomissâo di Pós Graduach

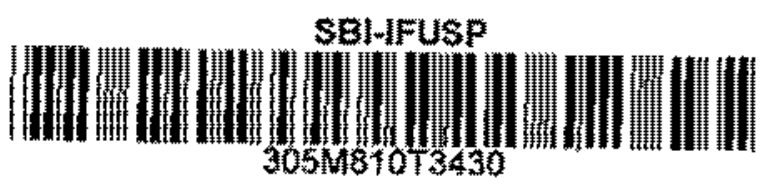




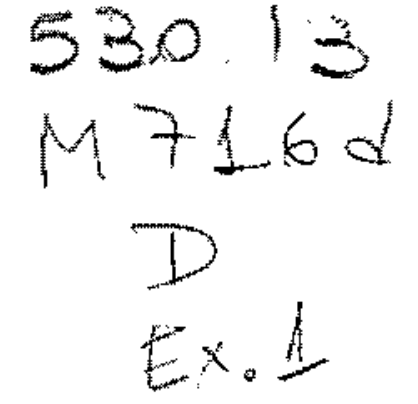

FICHA CATALOGRÁFICA

Preparada pelo Serviço de Biblioteca e Informação do Instituto de Fisica da Universidade de São Paula

Mokarzel, Sonia Gerail

Decoerência e Dissipacăo em Sistemas Quànticos Acoplados; Técnicas e Aplicaçoes. São Paulo, 2000.

Tese (Doutoramento) - Universidade de Săo Paulo. Instituto de Fisica - Departamento de Física Matemática

Orientador: Profa. Dra. Maria Carolina Nemes

Area de Concentraçäo: FIsica Quântica

Unítemos: 1. Decoerência e Dissipaçâo;

2. Algebra de Lie;

3. Mecanica Estatistica de nâo Equilibrio;

4. Desacoplamento de Superoperadores. 


\section{Agradecimentos}

Preciso começar agradecendo a minha orientadora Maria Carolina Nemes, năo por obrigaçă, mais sim por ela ter me proporcionado enome alegria e prazer ao permitie a realização deste trabalho e por saber valoriaar de maneira incomparável todo potencial de seus alunos. Aprendi muito com isto.

Obrigada "Carol"!

A minha família pelas inúmeras oraçöes e principalmente aos meus pais pela vida que me deram.

Obrigada "tia Maria, Madame, Bela, Ceclia, Neguinha, Leta, Zinha, Tonhäo, Guco, Alessandra, Solange"!

Ao prof. A.F.R de Toleto Piza, que me iluminou con suas ideias, me presenteou com seus ensinamentos e principalmente por presenciar em sua pessoa um modelo vito e real de integridada humana. Obrigada!

Ao Ji il Küm pela dedicaçăo e interesse no acompanhamento e esclarecimentos durante todo o trabalho, e pelo ser Humano Maravilhoso que é. Obrigada!

Ao Alessandro Marques pelas incontáveis horas que debruçou sobre meu computador e meus programas para que este trabalho pudesse sair. Obrigada!

A Catlarina M. Gumarates Penha, que com seu exermplo de vida me deu forças para prosseguir nos momentos mais dificeis. Obrigada!

A Maria Regìa Guarnieri e suas irmäs "Já e Lú" que com paciencia (e que paciêncial), carinho e dedicaçăo colocaram-se como ouvintes de minhas lamentaços e sofrimentos quando as "continhas" näo davam certo. Obrigada!

A Maria Helena Correia Alves por ter acreditado a todo momento primcipalmente por ter mantida acesa a chama de minha vontade de aprender. Obrigada!

$\grave{A}$ você que injustamente deixei de citar peço perdão e agradeco no fundo de minha alma por tudo que me proporcionoł e principalmente pela lição de humildade que está me dando agora. 


\section{Resumo}

Desenvolvemos no presente trabalho um tratumento perturbativo para a matriz densidade reduzida de torma sinilar a regra áurea de Fermi para espalhamento. Aplicarnos a teoria a vários exemplos e em particular reproduzimos os resultados experimentais obtidos no laboratório Kastler Brossel e obtivenos uma relação entre os tempos caracteristicos de dissipação e decoeréncia.

Por outro lado, desenvolvemos un modelo simples para duas moléculas interagindo com um reservatónio. Mostramos resultados surpreendentes quando tenos mais que dois substemas interagindo: no caso particular em que as moléculas estão num estado inicial coerente, bombardeadas por fontes de mesma intensidade, o estado assintotico apresenta uma concentração de energia no modo de menor frequểncia. Este resultado dá suporte a um modelo fenonenológico de sistemas biológicos onde a condensação de Bosé Einstein é produzida e o estado final também exibe uma concentração de energia no modo de frequiência mais baixa. 


\section{Abstract}

In the present work we developed a perturbative treatment of reduced density matrices which is similar in spirit to Fermi's Golden Rule for scattering. We applied the theory to several examples and in particular reproduced the results obtatien in the laboratory Kastler Brossel experiment quantifying the relation between decoherence and lissipation characteristic times.

On the other hand we developed a simple model for two molecules interacting through a reservoir. We show that rather surprising result may arise when we have nore hum two subsystens in interaction: in the particular case where both nolecules are initilly in coherent states, if they are pumped with the same strengh, he asymptotit state shows a concentraction of energy on the mode with smallest frequency. This result gives support to a phenomenological model for biological systems where at Bose Einstein condensation is predicted and the final state also exhibits a concentration of energy in the lowest frequency mode. 


\section{Sumário}

$\begin{array}{ll}\text { Introduçäo } & 7\end{array}$

1 Consideraçōes Gerais 9

1.1 Formulaçâo da Dinâmica Quântica . . . . . . . . . . 9

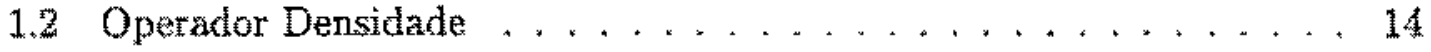

1.2 .1 Estado Puro Mistura Estatística . . . . . . . . . 16

1.2 .2 Evoluçào Temporal . . . . . . . . . . . 17

1.2.3 Operador Densidade Reduzido . . . . . . . . . . 18

1.3 Dinâmica reduzida . . . . . . . . . . . . . . . . 19

1.4 Decoerência, correlaçẫo e dissipaçăo . . . . . . . . 23

2 "Regra Aurea"para Decoerência e Dissipaçāo 25

2.1 Expansăo Perturbativa da Densidade Reduzida $\ldots \ldots \ldots \ldots, 25$

2.2 Acoplamento Linear a um banho de Osciladores . . . . . . . 26

2.3 Decoerência e Dissipaçäo de umá Superposiçäo Coerente . . . . . 30

3 Técnicas de Lie para Superoperadores 33

3.1 Algebra de Lie para Superoperadores . . . . . . . . . . . 33

3.1.1 Transformaçăo de Similaridade e Fórmula de Hausdórff ... 35

3.1.2 Fórmulas de Baker-Campbell-Hausdorff e de Zassenhaus . . 36

3.1 .3 Exemplo - O operador Deslocamento unitário de Glauber . . . 37

3.2 Desacoplamento e ordenarnento de operadores . . . . . . . . . 37

3.2 .1 Técnica matriclal . . . . . . . . . . . . 37

3.2 .1 Exemplo. . . . . . . . . . . . . . . 37

3.2.2 Técnica de Witschel e Técnica por derivaçäo de parâmetros . 38

3.2.2.1 Exemplo: 0 oscilador harmonico forçado dissipativo 40

4 Sistema aberto para dois osciladores 43

4.1 Deduçăo da equaçẫo mestra $\ldots \ldots \ldots \ldots \ldots \ldots \ldots \ldots$

4.2 Operador densidade para dois osciladores . . . . . . . . 54

4.3 Limite Assintótico - Aplicaçòes . . . . . . . . . . . . . . 58

4.4 Decoerência e Dissipação de uma Superposiçăo Coerente $\ldots \ldots .62$ 
A Evolução de um estado coerente $\quad 68$

A.1 Estados coerentes . . . . . . . . . . . . . . . . 68

A.2 Superoperadores agindo em un estado coerente ......... . . . . . . .

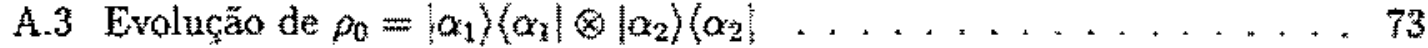

B Aplicaçäo da técruica $3.2 \times 2$ para dois osciladores $\quad 76$

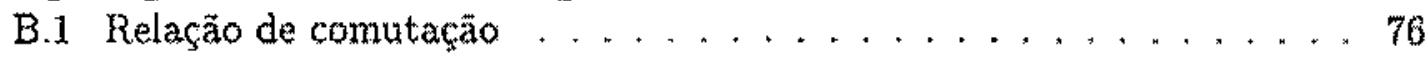

B.2 Desacoplamento de dois osciladores .............. 80

B.3 Cálculo dos coeficientes . . . . . . . . . . . . . . 97 


\section{Introdução}

O século que viu nascer a Mecànica Quântica estâ terminando com um vertiginoso progresso tecnológico que tem permitido nas últimas décadas testes contundentes de aspectos da interpretação da teoria quântica que foram assuntos de longas discussóes, debates e divergências ao longo do mesmo[22]. Um exemplo marcante $e$ o da existência de superposigōes coerentes de estados macroscopicamente distinguivejs, previsto pela Mecânica Quäntica e que implicaria necessariamente (uma vez reivindicado um caráter universal desta teoria) no aparecinzento do mesmo fenômeno a nivel macroscópico (paradoxo do "gato de Schrödinger "[13, 22, 39]). Una das soluçōes propostas para este problema foi a modificaşäo da própria equaçăo de Schrödinger [31] ou sua substituiçāo por equaçōes estocásticas $[11,17]$ ou ainda hamiltonianos dependentes do tempo $[4,37,38]$. A outra vertente que propöe a soluçāo atualmente mais aceita é a de considerar-se como sistema fechado o sistema de interesse mais - ambiente que o cerca (ou o aparelho de medida). Quantiza-se entảo o sistema composto de acordo con as regras de quantizaçäo usuais. Dentro deste contexto, a "não observacionalidade" de fenômenos de interferência a nivel macroscópico é então atribuido ao acoplamento entre o sistema de interesse e o ambiente que o cerca. $O$ mecanismo responsável pelo fenómeno é essencialmente quântico e conhecido como decoerência (perda de pureza) [13]. Recentemente, o grupo do laboratório francês Kastler - Brossel, em Paris, observol a evoluçäo temporal de uma supexposiçăo de estados coerentes numa cavidade supercondutora e mostrou que no caso em que a separação entre os estados que compöe a funç̧ấ de onda inicial se torna macroscópica, o tempo de decoerência torna-se muito menor do que qualquer outro tempo característico do sistema [6].

Uma primeira contribuiçăo deste trabalho é no sentido de caracterizar matématicamente o processo de decocrencia e o processo concomitante de dissipaçã̃o em geral. Para tanto, desenvolvemos uma teoria de perturbaçăo para densidades reduzidas, bastante similar ern espirito à regra ăurea de Fermi para o espalhanento. Em particular, aplicamos o formalismo ao experimento de Paris caracterizando as escalas de tempo de decoerencia e dissipaçäo e comprovando quantitativamente o resultado experimental. Usamos nossos resultados ainda para investigar outros casos de interesse.

Una outra vertente importante de investigaçăo atual relativa às bases da Mecảnica 
Quântica foi propiciada pela obseryaçāo em laboratónio de condensados bosônicos de átomos frios. Desde sua descoberta um enorme esforço teórico tem sido dedicado à sua compreensão [1].

O fenomeno de condensaçăo bosônica e correlaçöes de nuitos corpos nảo parece ser objeto de estudo exclusivo da Física. Já em $1968 \mathrm{H}$. Fröhlich [32] propöe um mecanismo de coerëncia de longo alcance em sisternas biológicos que resultaria num fenômeno desse típo também: a idéia principal ê que se moléculas com momento dipolar não nulo estão mergulhadas num reservatónio comum, vão trocar energia atrayés do reservatónto e, se energia for bombeada a essas moléculas, haverá transferễnia da mesna aos outros graus de liberdade presentes no processo de tal forma que o estado estacionário que será atingido apresentará um excesso de energia canalizado para um único modo (exatamente como no fenơneno de condensaçäo de Bose - Einstein). Esse modo é o modo de menor frequêtncia.

Obviamente a soluçầo completa desse problema é extremamente complicada. No entanto, a conștrução de um modelo esquemático com duas moléculas interagindo com um reservatório é possivel de ser mostrado e, embora esteja longe de poder exibir o fenömeno de condensação bosônica, permite estudar alguns mecanismos importantes para o mesmo de forma analítica. Com essa motivação construínos entâo un modelo de dois osciladores acoplados via reservatório. Do ponto de vista da Mecânica Quântica este é um problema interessante em sí, por envolver nais do que dois subsistemas en interafão. Pudemos observar que as propriedades de transferência de energia, por exemplo, podem revelar comportamentos inesperados e surpreendentes. Em particular mostramos que, sendo os osciladores inicialnente em estados coerentes, bombeados por fontes de mesma intensidade, (essa condiçāo pode ser eventualmente relaxada) o estado assintótico será tal que o oscilador de menor freqüencia concentrará a maior quantidade de energia.

A resolução completa e analitica deste problema foi possivel graças a uma extenção de técnicas de algebra de Lie para superoperadores $\{14,24,29\}$. Esta técnica foi originalmente proposta e usada para o calculo de correlaçôs. Ela pode ser generalizada para sistemas linearmente acoplados $€$, como fizemos, para o calculo de densidades reduzidas.

Assim como propriedades de transferencia de energia em sistemas que envolvem mais do que dois graus de liberdade sezo surpreendentes, as propriedades de decoerência também constituem um estudo muito rico dinämicamente. Resultados preliminares indicam que há também "transferência de coerêncià"entre os subsistemas em questầo. 


\section{Capítulo 1}

\section{Considerações Gerais}

Este capitulo ể um compêndio das ferramentas necessảrias para o desen volvimento da tese. Serve também ao propósito de estabelecer a linguagem que será usada até o fim do nosso trabalho.

\subsection{Formulação da Dinâmica Quântica}

O estado de un sistema quântico é completamente especificado por uni elemento de um espaço vetorial. Na representação de coordenadas, os vetores são dados pelas funçöes de onda de quadrado integrável cujo módulo ao quadrado mede a probabilidade de encontrar a partícula no ponto " $x$ ". Para obtermos a evoluçăo de um sistema quântico "postula-se" a existência de um hamiltoniano, $\mathbf{H}$, e que a evoluçăo de un estado $|\psi(t)\rangle$ seja de acordo com a equaçâo de Schródinger.

$$
\left.\left.i f \frac{d}{d t} \mid \psi(t)\right)=\mathrm{H} \mid \psi(t)\right\}
$$

onde $\mathrm{H}$ é un observảvel e portanto hermiteano. Supondo que $H$ năo dependa explicitamente do tempo, entầ, formalmente podemos escrever:

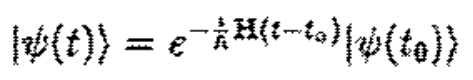

onde $\mid \psi\left(t_{0}\right)$ é o estado do sistema no instante $t_{0}$. Seja

$$
\mathrm{U}\left(t, t_{0}\right) \equiv e^{-\frac{3}{t_{1}} \mathrm{H}\left(t-\mathrm{k}_{\mathrm{s}}\right)}
$$

Como Hé hermiteano, entāo

$$
\mathrm{U}^{i}\left(t, t_{0}\right)=e^{\frac{1}{\hbar} \mathrm{H}\left(t-t_{0}\right)}=\mathrm{U}^{-1}\left(t, t_{0}\right),
$$

ou seja, $U\left(t, t_{0}\right) \hat{e}$ urn operador unitário. Ele possui as seguintes propriedades notáveis: 
1. Conserva a condição de normalizaçäo do vetor de onda no tempo, satisfaz a equação

$$
i \hbar \frac{d}{d t} \mathbf{U}\left(t_{*} t_{0}\right)=\mathbf{H} \mathbf{U}\left(t_{1} t_{0}\right), \text { com } \mathbf{U}\left(t_{2}, t_{1}\right)=1
$$

2. Satisfaz a propriedade de grupo

$\mathrm{U}\left(t_{1} t_{1}\right) \mathrm{U}\left(t_{1}, t_{0}\right)=\mathrm{U}\left(t_{1} t_{0}\right)$, propriedade que resulta da evoluçăo matematica determinista da equaçāo de Schrödinger.

No caso de $\mathbf{H}$ depender explicitamente do tempo, um operador de evoluçăo pode ser definido generalizando a equação 1.4

$$
i \hbar \frac{d}{d t} \mathbf{U}\left(t, t_{0}\right)=\mathbf{H}(i) \mathrm{U}\left(t, t_{0}\right) \text { com } \mathbf{U}\left(t_{0}, t_{0}\right)=\hat{i} \text { e } \mathbf{H}(t) \text { hermiteano. }
$$

Neste caso, no entanto, o Hamiltoniano $\mathbf{H}(t)$, en geral, năo comuta consigo mesmo en tempos diferentes, ou seja,

$$
\left[\mathbf{H}_{1}\left(t_{1}\right), \mathbf{H}_{2}\left(t_{2}\right)\right] \neq 0 \text { se } t_{1} \neq t_{2}
$$

Supondo que $\mathbf{H}_{1}$ e $\mathbf{H}_{2}$ sejam independente do tempo nos intervalos $t_{0}<t_{1}<t_{0}+\Delta t$ et $t_{0}+\Delta t<t_{2}<t_{0}+2 \Delta t$ respectivamente, entäo

$$
\begin{aligned}
\mathrm{U}\left(t_{0}+2 \Delta t, t_{0}\right) & =\mathrm{U}_{2}\left(t_{0}+2 \Delta t, t_{0}+\Delta t\right) \mathrm{U}_{1}\left(t_{0}+\Delta t, t_{0}\right) \\
& =e^{-\frac{t_{h}}{H_{2}} \Delta t_{2}} e^{-\mathrm{H}_{2} \Delta t} \neq \mathrm{e}^{-\frac{t_{h}}{\hbar}\left(\mathrm{HI}_{2}+\mathrm{H}_{2}\right) \Delta t}
\end{aligned}
$$

pois, $\left[\mathrm{H}_{1}\left(t_{1}\right), \mathrm{H}_{2}\left(t_{2}\right)\right] \neq 0$, mostrando que $\mathrm{U}\left(t_{1} t_{0}\right) \neq e^{-\frac{i}{\hbar} \int_{\mathrm{t}_{0}}^{t} \mathrm{H}\left(t^{\prime}\right) d t^{\prime}}$ que seria de fato a solução correta se $\mathbf{H}(t)$ fosse una funçẫo numérica.

A solução que leva em conta a não comutatividade do Hamiltoniano em tempos diferentes poderá ser pensada em termos de uma sucessäo de transformaçōes unitárias infinitesimais que avançam no tempo em intervalos dt durante os quais a variação de $\mathbf{H}(t)$ é desprezivel

$$
\mathbf{U}(t+d t)=\mathbf{i}-\frac{1}{n} \mathbf{H}(t) d t+o\left(d t^{2}\right)
$$

Cuja representação integral é dada por [26]

$$
\mathrm{U}\left(t, t_{0}\right)=1-\frac{i}{\hbar} \int_{t_{0}}^{t} \mathrm{H}\left(t^{t}\right) \mathrm{U}\left(t^{z}, t_{0}\right) d t^{t}
$$

Seja $\mathrm{H}$ dependente on independente do tempo, $\mathrm{U}\left(t, t_{0}\right)$ deve ser unitário s satisfazer a propriedade de grupo. No que segue consideraremos o caso de hamiltonianas independentes do tempo, por simplicidade.

A evolução unitária pode ser imaginada como uma "rotaçăo" continua do vetor de estado no espaço de Hilbert, $\mathcal{E}$, que goza das seguintes propriedades: 
1. O produto escalar de dois vetores no espaço de Hilbert, $\mathcal{E}$, permanece constante se ambos forem submetidos à mesma evoluçāo unitária

$$
\langle\mathbf{U} \psi \mid \mathbf{U} \phi\rangle=\left\langle\psi \mid \mathbf{U}^{\dagger} \mathbf{U} \phi\right\rangle=\langle\psi \mid \phi\rangle
$$

2. Uma evolução unitária leva estados ortogonais, $\phi_{n_{i}} \phi_{n_{2}}$ onde $n_{i} n_{j}$ sāo indices discretos arbitrários, à estados ortogonais

$$
\left\langle\mathcal{U} \phi_{n_{i}} \mid \cup \phi_{n_{2}}\right\rangle=\left\langle\phi_{n_{i}} \mid U^{\dagger} U \phi_{n_{j}}\right\rangle=\left\langle\phi_{n_{1}} \mid \phi_{n_{j}}\right\rangle=\delta_{n_{1} n_{j}} \text { se }\left\{\left|n_{i}\right\rangle\right\} \text { for ortogonal. }
$$

3. Transforma um vetor de estado $|\psi\rangle$ em $\left|\psi^{\prime}\right\rangle$, segundo a regra $\left|\psi^{\prime}\right\rangle=\mathbf{U}|\psi\rangle$. Com isto, a evoluçäo de un estado $|\psi(t)\rangle(1.1)$, pode ser descrita por uma tranformação unitảiria

$$
|\psi(t)\rangle=\mathbf{U}\left(t, t_{0}\right)\left|\psi\left(t_{0}\right)\right\rangle \text { com a condição inicial, para } t=t_{0}, \mathbf{U}\left(t_{0}, t_{0}\right)=\hat{1}
$$

Deste modo, a probabilidade de encontrar o estado $\left|\psi\left(t_{0}\right)\right\rangle$ no vetor de estado $\left|\phi_{n}\right\rangle$ é:

$$
p_{n}\left\{t_{n}\right)=\left.\left|\left\langle\phi_{n}\right| \psi\left(t_{0}\right)\right\}\right|^{2}
$$

evolù dinamicanente no tempo cono:

$$
\left.p_{n}(t)=\left|\phi_{n}\right| \psi(t)\right\rangle\left.\right|^{2}=\left|\left\langle\phi_{n}\left|U\left(t_{s} t_{0}\right)\right| w\left(t_{0}\right)\right\rangle\right|^{2}
$$

4. Transforma un operador hermiteano, $O$, escrito na base $\{|n|\}, O=\sum O_{n m}|n|\langle m|$, em um operador $\mathbb{O}^{\mathrm{O}}$ na base $\left.\{|m|\rangle^{\prime}\right\}$, onde $|n\rangle^{\prime}=\mathrm{U}|n\rangle$, segundo a regra

$$
\mathfrak{O}=\sum O_{n m}|n\rangle^{\prime}\left(\left.m\right|^{*}=\sum O_{n m} \mathrm{U} \mid n\right)\left(m \mid \mathrm{U}^{\dagger}=\mathrm{U}\left(\sum \mathrm{O}_{n m}|n| m \mid\right) \mathrm{U}^{\dagger}=\mathrm{UOU}^{\dagger}\right.
$$

No caso geral, a evoluçäo no tempo do valor médio de qualquer grandeza devido à evoluçẫo do vetor de estado, pode ser representada por:

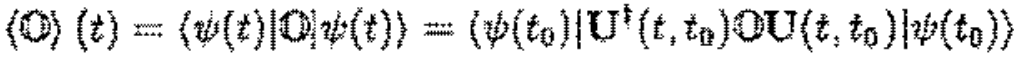

$$
\begin{aligned}
& =\left\langle\left(t\left(t_{0}\right)\left|O_{H}(t)\right| \psi^{\prime}\left(t_{0}\right)\right),\right. \text { onde }
\end{aligned}
$$

$\mathrm{O}_{H}(t)=\mathrm{U}^{\dagger}\left(t, t_{0}\right) \mathrm{OU}\left(t, t_{0}\right)$ th chamada representaçäo de Heisenterg do operador. Nestá representaçăo o operador evolui com o tempo e o vetor de estado não varia no tempo. Na representaçäo Schröhinger, a evoluçào temporal é atribuida ao vetor de estado mantendo-se os operadores fixos no tempo.

A equivalência das duas representaçôes é estabelecida pela equação 1.5 , ou seja,

$$
\begin{aligned}
& \mathbb{O}_{H}(t)=\mathbf{U}^{\dagger}\left(t, t_{0}\right) \mathbb{O}_{S}\left(t_{0}\right) \cup\left(t, t_{0}\right), \quad \text { ou ainda, } \\
& \left|\psi_{H}\left(t_{0}\right)\right\rangle=\mathbf{U}^{\dagger}\left|\psi_{S}(t)\right\rangle=\mathbf{U}^{\dagger} \mathbf{U}\left|\psi\left(t_{0}\right)\right\rangle=\left|\psi\left(t_{0}\right)\right\rangle .
\end{aligned}
$$


A evoluçāo temporal dos operadores na representaçâa de Heisenberg, é dada por:

$$
\begin{aligned}
& \frac{d}{d t} \mathrm{O}_{H}(t)=\left(\frac{d}{d t} \mathrm{U}^{\dagger}\right) \mathrm{O}_{S} \mathrm{U}+\mathrm{U}^{\dagger}\left(\frac{\partial}{\partial t} \mathrm{o}_{S}\right) \mathrm{U}+\mathrm{Uto}_{S}\left(\frac{d}{d t} \mathrm{U}\right) \\
& =+\frac{i}{n} \mathrm{U}^{\dagger} \boldsymbol{H}_{S} \mathrm{O}_{S} \mathrm{U}+\mathrm{U}^{\mathrm{H}}\left(\frac{\partial}{\partial t} \mathrm{O}_{S}\right) \mathrm{U}-\frac{i}{h} \mathrm{U}_{S} \mathrm{O}_{S} \mathrm{U} \\
& =-\frac{i}{\hbar}\left\{-\left(\mathrm{U}^{\dagger} \mathrm{H}_{S} \mathrm{U}\right)\left(\mathrm{U}^{\dagger} \mathrm{O}_{S} \mathrm{U}\right)+\mathrm{U}^{\dagger}\left(\frac{\partial}{\partial t} \mathrm{O}_{S}\right) \mathrm{U}+\left(\mathrm{U}^{\dagger} \mathrm{O}_{S} \mathrm{U}\right)\left(\mathrm{U}^{\dagger} \mathbf{H}_{S} \mathrm{U}\right)\right\} \\
& =-\frac{i}{\hbar}\left\{\left[\mathbb{Q}_{H}(t), \mathbf{H}_{H}\right]+i \hbar\left(\frac{\partial}{\partial t} \mathbf{Q}_{S}\right)\right\} \\
& \downarrow \\
& i f \frac{d}{d t} \mathrm{O}_{H}(t)=\left[\mathrm{O}_{H}(t), \mathbf{H}_{H}\right]+i \hbar\left(\frac{\partial}{\partial t} \mathrm{O}_{S}\right)
\end{aligned}
$$

onde $\mathbf{H}_{H}=\mathbf{H}_{S}=\mathbf{H}$.

Se o operador $\mathbb{O}$ não depender explicitamente do tempo, então

$$
i \hbar \frac{d}{d t} \mathbb{O}_{H}(t)=\left[\mathbb{O}_{H}(t), \mathbf{H}_{H}\right] .
$$

As descriçōes de Heisenberg e de Schrödinger não esgotam as alternativas de formulação para a dinâmica quântica. $\hat{\mathrm{E}}$ comum estudar sistemas cujo hamiltoniano, independente do tempo, pode ser decomposto em uma parte não interagente, $\mathbf{H}_{0}, \mathrm{e}$ uma parte que descreve a interaçăo do sistema com outro, $\mathrm{H}_{\text {in: }}$. Neste caso, em que $\mathbf{H}_{\text {toint }}=\mathbf{H}=\mathbf{H}_{0}+\mathbf{H}_{\text {int }}$ é conveniente usar a repressentaçăo de interoção introduzida por Dirac, que em certo sentido é intermediaria entre a representaçâo de Schrödinger (evoluçào do vetor de estado no tempo em relaçăo apenas a $\mathbf{H}_{\text {ina }}$ ) e de Heisenberc (evolução dos operadores no tempo em relaçäo apenas a $\mathrm{H}_{0}$ ). Nesta representação, define-se, além do já definido operador unitário da equaçăo 1.3, um operador de evolução também unitário, mas associado sonente a $\mathrm{H}_{0}$

$$
\mathbf{U}_{0}\left(t_{3}, t_{0}\right)=e^{-\frac{1}{x} \boldsymbol{H}_{0}\left(t-t_{0}\right)}
$$

Deste modo, evoluçāo dinâmica da probabilidade calculada no tempo, $t$ poderá ser escrita por:

$$
\begin{aligned}
& p_{n}(t)=\mid\left\langle\left.\phi_{n}|\psi(t)|\right|^{2}=\left|\phi_{n}\right| \cup\left(t, t_{0}\right)\left|\psi\left(t_{0}\right)\right|^{2}\right. \\
& \left.=\left|\left(\omega_{n}\right) \mathrm{U}_{0}\left(t, t_{0}\right) \mathrm{U}_{0}^{\dagger}\left(t, t_{0}\right) \cup\left(t, t_{0}\right)\right| \omega_{(}\left(t_{0}\right)\right)\left.\right|^{2} \\
& =\left|\left\langle\phi_{n}\left|\mathbf{U}_{0}\left(t, t_{0}\right)\right| \vec{\psi}(t)\right\rangle\right|^{2}
\end{aligned}
$$


que pode ser interpretada em termos do produto escalar do autovetor dependente do tempo $\mathrm{U}_{0}^{\dagger}\left\langle t, t_{0}\right\rangle\left|\phi_{n}\right\rangle$ com o vetor de estado dependente do tempo, definido na representaçäo de interação, por

$$
\left.|\tilde{\psi}(t)\rangle=U_{0}^{t}\left(t_{0} t_{0}\right) \mid \psi_{s}(t)\right)
$$

A evoluçăo deste estado é dada por:

$$
\begin{aligned}
\frac{d}{d t}|\widetilde{\psi}(t)\rangle & \left.=\left(\frac{d}{d t} \mathrm{U}_{0}^{\dagger}\left(t, t_{0}\right)\right) \mid \psi_{s}(t)\right)+\mathrm{U}_{0}^{\dagger}\left(t, t_{0} \mid\left(\frac{d}{d t} \mid \psi_{s}(t)\right)\right. \\
& =+\frac{i}{\hbar} \mathrm{U}_{0}^{\dagger}\left(t, t_{0}\right) \mathbf{H}_{0}\left\{\psi_{s}(t)\right)-\frac{i}{\hbar} \mathrm{U}_{0}^{\dagger}\left(t, t_{0}\right) \mathbf{H}\left|\psi_{s}(t)\right\rangle \\
& =-\frac{i}{\hbar} \mathrm{U}_{0}^{\dagger}\left(t, t_{0}\right) \mathrm{H}_{\mathrm{in} t}\left|\psi_{s}(t)\right\rangle \\
& =-\frac{i}{\hbar}\left(\mathrm{U}_{0}^{\dagger}\left(t, t_{0}\right) \mathrm{H}_{\text {int }} \mathrm{U}_{0}\left(t, t_{0}\right)\right)\left(\mathrm{U}_{0}^{\dagger}\left(t, t_{0}\right)\right)\left|\psi_{s}(t)\right\rangle \\
& \downarrow \\
\frac{d}{d t}|\widetilde{\psi}(t)\rangle & =-\frac{i}{\hbar} \tilde{\mathbf{H}}_{i n t}(t)|\widetilde{\psi}(t)\rangle
\end{aligned}
$$

onde

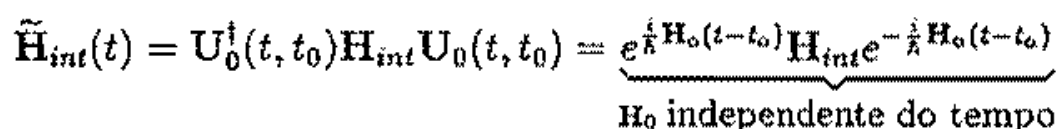

note que o vetor de estado na representação de interação, $|\tilde{\psi}(t)\rangle$, evolui devido apenas a hamiltoniana de interação dos sistemas.

Por outro lado, a evoluçăo no tempo de qualquer variável dinâmica 0 , de acordo com a equaçå̉o 1.5, nos leva a:

$$
\begin{aligned}
\langle 0\rangle(t) & =\langle\psi(t)|\Phi| \psi(t)\rangle=\left\langle\tilde{\psi}(t)\left|\cup_{0}^{\dagger}\left(t, t_{0}\right) \cup U_{0}\left(t, t_{0}\right)\right| \tilde{\psi}(t)\right\rangle \\
& =\langle\tilde{\psi}(t)|\tilde{O}(t)| \tilde{\psi}(t)\rangle
\end{aligned}
$$

ande

$$
\widetilde{O}(t)=U_{0}^{\dagger}\left(t, t_{0}\right)\left(U_{0}\left(t_{t}, t_{0}\right)\right.
$$

define um operador na representação de interação.

A evoluçäo temporal dos operadores na representaçầo de interaçäo e dado por:

$$
\begin{aligned}
i \hbar \frac{d}{d t} \tilde{\mathrm{O}}(t) & =i \hbar \frac{d}{d t}\left(\mathrm{U}_{0}^{\dagger}\left(t, t_{0}\right) \mathrm{U}_{0}\left(t, t_{0}\right)\right) \\
& =-\mathrm{H}_{0} \widetilde{\mathrm{O}}(t)+i \hbar \mathrm{U}_{0}^{\dagger}\left(t, t_{0}\right)\left(\frac{d_{0}}{d t}\right) \mathrm{U}_{0}\left(t, t_{0}\right)+\tilde{0}(t) \mathrm{H}_{0}
\end{aligned}
$$


Como o näo depende explicitamente do tempo,entäo

$$
i \hbar \frac{d}{d t} \tilde{\mathbb{O}}(t)=\left[\tilde{\mathbb{O}}(t), \mathbf{H}_{0}\right]
$$

Se compararmos a equação anterior à equaçâo 1.7 observamos que a evoluçăo temporal dos operadores na representaçāo de interação, $\tilde{\omega}(t)$, se dá devido apenas a hamiltoniana intrinseca do sistema.

\subsection{Operador Densidade}

Descrever um sistema fisico através de um vetor de estado näo te forma mats geral. Un formalismo geral que permite descrever qualquer sistema físico o fomalismo da matriz densidade, que caracteriza o sistema hísico por um conjunto de vetores

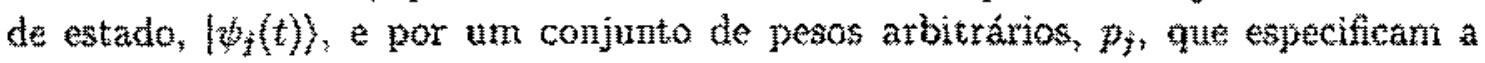
contribuiçẵo relativa de cada um de seus estados.

Cada um dos estados possiveis, $\left.\mid \psi_{j}(t)\right)$, que e nomalizado mas nato nectessatiamente ortogonal, poderá ser expandido nurna base, $\phi_{n}$ onde $\left\langle\phi_{n} \mid \phi_{m}\right\rangle=\delta_{n n} \sum_{m}\left|\phi_{n}\right|\left\langle\phi_{n}\right|=$ 1 (relaşäo de completeza).

$$
\left|\psi_{j}(t)\right\rangle=\sum_{n} C_{n}^{(j)}(t)\left|\phi_{\mathrm{n}}\right\rangle \Longrightarrow C_{n}^{(j)}(t)=\left\langle\phi_{n} \mid \psi_{j}(t)\right\rangle
$$

Assim, podemos escrever a matriz densidade por:

$$
\left.\rho_{s r}=\sum_{j} p_{j}\left\langle\phi_{s} \mid \psi_{j}(t)\right\rangle\left\langle\psi_{j}(t)\right| \phi_{r}\right)=\sum_{j} C_{s}^{(j)}(t) C_{r}^{r(j)}(t)
$$

Se $A$ e um observatul com elementos de matriz $A_{m m}=\left\langle\phi_{m}|A| \phi_{n}\right\rangle$ entato, o valor médio do A no instante t será:

$$
\begin{aligned}
& (A)(t)=\sum_{i} p_{i}\left(w_{j}(t)\left(\mathrm{A} \mid w_{j}(t)\right)\right. \\
& =\sum_{j} p_{3}\left(w_{3}(t)|\mathrm{A}| \sum_{n} \mid \phi_{n}\right)\left(\phi_{n} \mid w_{j}(t)\right. \\
& =\sum_{n} \sum_{j} p_{j}\left(\psi_{j}(z)|A| \phi_{n}\right)\left(\phi_{n} \mid \psi_{j}(t)\right) \\
& =\sum_{n}\left(\left\langle\omega_{n}\right| \sum_{j} p_{j}\left(\psi_{j}(t)\right)\right)\left\langle\psi_{j}(t)|A| \phi_{n}\right) \\
& =\sum_{n}\left(\phi_{n} \mid\left(\sum_{j} p_{j}\left|\psi_{j}(t)\right\rangle\left(\psi_{j}(t)\right)\right) A \mid \phi_{n}\right)
\end{aligned}
$$


ou seja

$$
\text { (A) }(t)=\operatorname{Trp}(t) A
$$

onde

$$
p(t)=\sum_{i} p_{j}\left|\psi_{j}(t)\right\rangle\left\langle\psi_{j}(t)\right| \text { é o operador densidade. }
$$

Fortanto o operador densidade é a generalizaçăo do vetor de sstado e a matriz densidade $\hat{e}$ a representação do operador densidade en certa base. O operador densidade obedece às seguintes propriedades:

1. o hermiticidade

$$
\begin{aligned}
\rho^{j} & \left.=\left(\sum p_{j}\left|\psi_{j}(t)\right\rangle \psi_{j}(t)\right)\right)^{\prime} \\
& \left.=\sum_{j} p_{j}^{*} \mid \psi_{j}(t)\right)\left(\psi_{j}(t) \mid\right.
\end{aligned}
$$

Como

$$
p_{j}=p_{j}^{*} \text { pois } p_{j} \text { é real, }
$$

então

$$
\rho^{\dagger}=p(t)
$$

Conseqüentemente:

(a) O valor médio de qualquer observável ể real,

$$
\begin{aligned}
(\mathrm{A})^{*}(t) & =(\operatorname{Tr} \rho(t) \mathbf{A})^{*}=\operatorname{Tr}(\rho(t) \mathbf{A})^{\dagger} \\
& =\operatorname{Tr}(\rho(t))^{\dagger}(\mathbf{A})^{\dagger}=\operatorname{Tr} \rho(t) \mathbf{A} \\
& =(\mathbf{A})(t) .
\end{aligned}
$$

(b) O operador densidade pode ser diagonalizado por uma transformação unitária

$$
\begin{aligned}
& p^{\prime}(t)=U \rho(t) \mathrm{U}^{z} \\
& \Longrightarrow \quad \operatorname{Tr} \rho^{*}(t)=\operatorname{Tr} \mathrm{U} \rho(t) \mathrm{U}^{\dagger}=\operatorname{Tr} \mathrm{U}^{\dagger} \mathrm{U} p(t)=\operatorname{Tr} p(t) \\
& \Longrightarrow \text { o traço de um operador é un invariante a mudança de representação. }
\end{aligned}
$$




\section{2. $\diamond$ Positividade semi definida}

Seja um estado qualquer $|v\rangle$, entäo

$$
\begin{aligned}
\langle v|\rho(t)| v\rangle & =\left\langle v\left|\sum_{j} p_{i}\right| \psi_{j}(t)\right)\left(\psi_{j}(t)|v\rangle\right. \\
& =\sum_{j} p_{j}\left(v\left|\psi_{j}(t)\right|\left(\psi_{j}(t) \| v\right\rangle\right. \\
& =\left.\sum_{j} p_{j}\left|\langle v| \psi_{i}(t)\right)\right|^{\frac{2}{2}} \geq 0 \\
& \downarrow \\
& \rho(t) \geq 0 .
\end{aligned}
$$

3. $\diamond \operatorname{Tr} p^{2}(t) \leq 1$

$$
\begin{aligned}
\operatorname{Tr} \rho^{2}(t) & =\sum_{n}\left\langle\phi_{n}\left|\left(\sum_{j} p_{j}\left|\psi_{j}(t)\right\rangle\left\langle\psi_{j}(t)\right|\right)\left(\sum_{k} p_{k}\left|\psi_{k}(t)\right| \psi_{k}(t)\right)\right| \phi_{n}\right\rangle \\
& =\sum_{j} p_{j} \sum_{k} p_{k} \sum_{n}\left\langle\psi_{k}(t) \mid \phi_{n}\right\rangle\left\langle\phi_{n} \mid \psi_{j}(t)\right\rangle\left\langle\psi_{j}(t) \mid \psi_{k}(t)\right\rangle
\end{aligned}
$$

da relação de completeza, $\sum_{n}\left|\phi_{n}\right\rangle\left\langle\phi_{n}\right|=1$, teremos

$$
\operatorname{Tr} p^{2}(t)=\sum_{j} p_{i} \sum_{k} p_{k} \mid\left(\psi_{k}(t)\left|\psi_{j}(t)\right|^{2}\right.
$$

Como

$$
\left|\left\langle\psi_{k}(t) \mid \psi_{j}(t)\right\rangle\right|^{2} \leq 1 \text { e } \sum_{k} p_{k}=1 \Rightarrow \sum_{k} p_{k} \mid\left(\left.t_{k}(t)\left|\psi_{j}(t)\right|\right|^{2} \leq 1 \text { para } \forall k_{k}\right.
$$

entẫo

$$
\operatorname{Tr} p^{2}(t) \leq \sum_{j} p_{j}=1
$$

\subsubsection{Estado Puro e Mistura Estatística}

O operador densidade pode ser visto como uma distribuiçấo muito grande de siste. mas idênticos, ensemble, preparados de tal forma que cada fração $p_{j}$ está no estado $\left|w_{i}(b)\right\rangle$ 
Se considerarmos um número muito grande de sistemas idênticos, todos preparados num mesno estado quântico, entầo

$$
p_{j}=1 \forall j \notin \sum_{j} p_{j}\left|\psi_{j}(t)\right\rangle\left\langle\psi_{j}(t)|=| \psi\right\rangle\langle\psi|
$$

Um tal conjunto ê denominado estado puro onde, o operador densidade passa a ser um projetor e conseqüientemente idempotente, ou seja,

$$
\rho(t)=|\psi|\left\langle\langle\psi| \text { (projetor) } \Longrightarrow \operatorname{Tr} \rho^{2}(t)=1\right. \text { (idempotente) }
$$

Desta forma é possível interpretar os autovalores de $\rho$ como probabilidades de que 0 sistema, cujo estado é descrito por p, se encontre no autovetor correspondente a esse autovalor. Tais probabilidades săo chamadas probabilidades de ocupaçäo associadas aos respectivos vetores de estado.

Potanto, uma maneira necessária e suficiente para se medir a purexa de um estado, que em laboratorio é sempre un procedimento delicado de se obter, é verficar se

$$
p^{2}(t)=p(t) \text { ou se } \operatorname{Tr} p^{2}(t)=\operatorname{Tr} p(t)=1
$$

Caso contrário teremos uma mistura estatística, onde

$$
\rho^{2}(t) \neq \rho(t) \text { e } \operatorname{Tr} \rho^{2}(t) \leq 1
$$

A diferença entre estados puros e mistura estatistica está relacionada a medida da "desordem" do sistema [25] através da entropia de Boltzman-von Newman [35]

$$
S_{B}=-\operatorname{Tr}(\rho \ln \rho)
$$

ou através da entropia linear $[5,13]$

$$
\delta(t)=T r\left(p-p^{2}\right) \text { defeito de idempotënaia }
$$

\subsubsection{Evolução Temporal}

Derivando-se o operador densidade, definido na equaçāo 1.12 , em relação ao tempo, obtemos

$$
\frac{d}{d t} \rho(t)=\sum_{j} p_{j}\left(\frac{d}{d t}\left|\psi_{j}(t)\right\rangle\right)\left\langle\psi_{j}(t)\left|+\sum_{j} p_{j}\right| \psi_{j}(t)\right\rangle\left(\frac{d}{d t}\left\langle\psi_{j}(t)\right|\right)
$$

Se o ensemble não é perturbado, cada um dos sistemas evolui de acordo com a equação de Schrödinger 1.1, cujo hermiteano conjugado é

$$
-i \hbar \frac{d}{d t}\langle\psi(t)|=\langle\psi(t)| \mathbf{H}
$$


Corn isto,

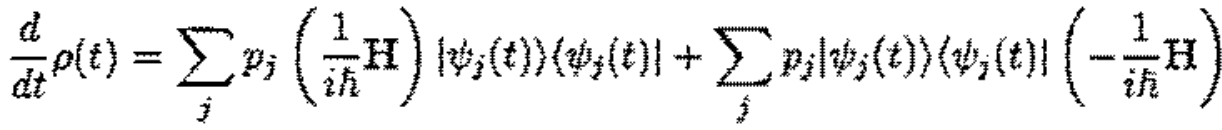

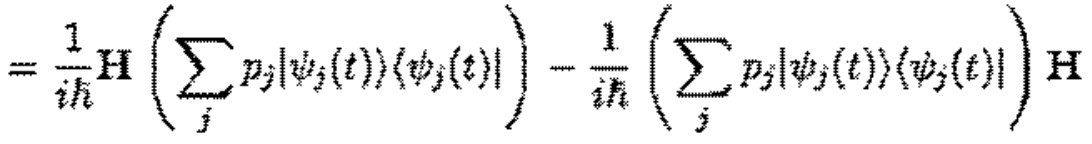

$$
\begin{aligned}
& =\frac{1}{i h}(\mathbf{H} p(t)-p(t) H)
\end{aligned}
$$

teremos

$$
\frac{d}{d t} \rho(t)=\frac{1}{i n}\left[\mathbf{H}_{*} \rho(t)\right]
$$

Devido a analogia com a mecânăca clássica, esta equaço é conhecida como equaçäo de Liounille-von Netmann. Se defnizmos un superoperador linear

$$
\left.\mathcal{L}=\frac{1}{i \hbar} \cdot \boldsymbol{H}_{\mathrm{t}}\right)
$$

denominado superoperador liouvilliano (a notação (v) denota o superoperador), que atua sobre o operador densidade $\rho(t)$, podemos escrever a equaçäo de Liouville-von Neumann numa forma mais compacta, ou seja,

$$
\frac{d}{d t} p(t)=E \rho(t)
$$

\subsubsection{Operador Densidade Reduzido}

Conforme já mencionamos, é comum tstudar sistenuas cujo haniltoniano pode ser decomposto numa parte intrinseca, $\mathrm{H}_{0}$ e numa parte de interaçăo, $\mathrm{H}_{\text {int }}$ Para podermos estudar os subsistemas deste sistema composto, através do operador densidade, temos que definir um operador densidade para cada un dos subsistemas.

Para tanto, vamos considerar um sistema composto, a 4 b + +..., formato pelos subsistemas $a, b, \ldots$ onde os estados de un sistema composto säo kstociados a vetores produto no espaço de Hibert, correspondentas hos estados de cada um tos subsistemas, ou seja, o espaço de estado do sistema composto $\mathrm{f}$ produto tensorial $\mathcal{E}_{a+b+.}=\mathcal{E}_{a} \otimes \mathcal{E}_{b} \otimes \ldots$ dos espaços de estados tos subsistemas $a, b, \ldots$ gerados por

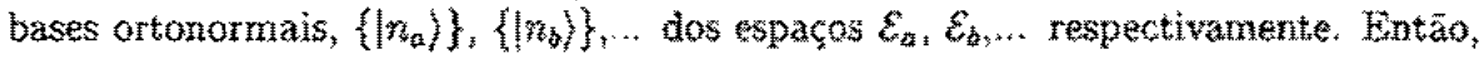


uma base do espaço produto, $\mathcal{E}=\mathcal{E}_{a+b+. .}$ constituida pelos vetores, também ortonormais da forma $\left.\left.\left\{\left|n_{a b . .}\right\rangle\right\}=|| n_{a}\right\}\right\}\left\{\left(\left|n_{b}\right\rangle\right\} \otimes \ldots\right.$

Assim, cualquer vetor, do espaco $\mathcal{E}_{\text {a }}$ por exemplo, pode ser escrito como

$$
\left.|a\rangle=\sum_{n a t} a_{n a t} \mid n_{n a}\right)
$$

e um vetor genérico no espaco produto, de dós subsistemas a e bor exemplo, será escrito por

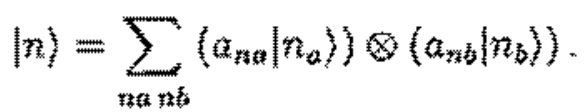

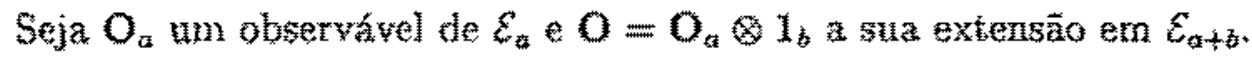

$O$ valor esperado de $O_{a}$ é dado por:

$$
\left(\mathrm{O}_{a}\right)=T r p \mathrm{O}
$$

onde $\rho$ é o operador densidade em $\varepsilon_{a+b}$ associado a sistena composto.

Assim:

$$
\begin{aligned}
\left\langle O_{\mathrm{a}}\right\rangle & =\sum_{n_{a} n_{b}}\left\langle n_{a} n_{b}|\rho O| n_{a} n_{b}\right\rangle \\
& =\sum_{n_{a} \text { abb }}\left\langle n_{a} n_{b}\left|\rho\left(O_{a} \otimes 1_{b}\right)\right| n_{a} n_{b}\right\rangle \\
& =\sum_{n_{a} n_{b}}\left\langlen _ { a } n _ { b } \left|\rho\left(O_{a}\left|n_{a}\right\rangle \otimes\left|n_{b}\right\rangle\right)\right.\right. \\
& =\sum_{n_{a}}\left\langle n_{a}\left|\left(\sum_{n_{b}}\left\langle n_{b}|\rho| n_{b}\right\rangle\right) O_{\alpha}\right| n_{a}\right\rangle .
\end{aligned}
$$

Definindo o operador densidade reduzzido do subsistema a por:

$$
p_{a} \equiv \sum_{n_{b}}\left\langle n_{b}|\rho| n_{b}\right\rangle \equiv T r_{b} \rho
$$

onde Tro denota o traco parcial no espaço $\mathcal{E}_{t,}$ podemos escrever

$$
\left(\mathrm{O}_{a}\right)=\sum_{n_{a}}\left(n_{\mathrm{a}}\left|p_{\mathrm{a}} \mathrm{O}_{\mathrm{a}}\right| n_{\mathrm{a}}\right\rangle=T r_{\mathrm{a}} p_{\mathrm{a}} \mathrm{O}_{\mathrm{a}}
$$

\subsection{Dinâmica reduzida}

A evoluçäo temporal do operator densidade reduaido, $p_{a}$, tada através do calculo do traço da equaça 1,15 


$$
\begin{aligned}
& \operatorname{Tr}_{b}\left(\frac{d}{d t} \rho(t)\right)=\frac{1}{i \hbar} \operatorname{Tr}_{b}([\mathbf{H}, \rho(t)]) \\
& \downarrow \\
& i \hbar \frac{d}{d t} \rho_{a}(t)=T r_{b}([\mathbf{H}, \rho(t)]) .
\end{aligned}
$$

Se näo houver interaçǟo entre os sistemas a e $b$ então o hamiltoniano total, $\mathrm{H}$, poderá ser decomposto en $\mathrm{H}=\mathrm{H}_{a}+\mathrm{H}_{b}$ e a evoluçào do operador densidade reduzido será unităria como a equaçäo 1.15 , ou seja,

$$
\hbar \frac{d}{d t} \rho_{\alpha}(t)=\left[\mathrm{H}_{\alpha}, \rho_{s}(t)\right] \text {. }
$$

Por outro lado quando há interaçăo entre os sistemas, $H_{\text {int, a }}$ evolução do operador densidade reduzido, $p_{a}$, não será em geral unitária. Formalmente, a maneira mais direta de ver isto consiste en considerarmos a densidade reduzida na representaçăo de orbitais naturais, i e, na representação em que ele é diagonal.Teremos entäo:

$$
\rho_{a}(t)=\sum_{j} p_{j}(t)\left|a_{j}(t)\right\rangle\left\langle a_{j}(t)\right|
$$

onde $p_{j}(t)$ representa a probabilidade de ocupaçäo do orbital no tempo $t$.

Considerando a derivada temporal de $\rho_{a}(t)$,

$$
i \frac{d}{d t} \rho_{\alpha}(t)=\left[\mathrm{h}(t), \rho_{\alpha}(t)\right]+\sum_{i}\left|o_{j}(t)\right\rangle\left(\frac{d}{d t} p_{j}(t)\right)\left\langle a_{j}(t)\right|
$$

onde $\mathrm{h}(t)$ e o operador hermiteano que caracteriza a transformaçäo unitária responsầvel pela evoluçăo dos orbitais naturais $\left\{a_{1}(t)\right)$, notamos claramente que 0 último termo do lado direito é uma contribuị̧ão não unităria pois, descreve a mudança dos autovalores como função do tempo. Estat dinẩmica jamais poderá ser caracterizada eñ ternos de um operador unitário.

E possivel encontrar uma relação formal entre $h(t), p_{j}(t)$ os ingredientes da dinâmica, $\boldsymbol{H}_{a,}, \mathrm{H}_{b} \mathrm{e} \mathrm{H}_{\mathrm{int}}$, [18], em termos de operadores de projeção e integrais de memória. Embora a estrutura da evoluçāo quântica de subsistemas interagentes esteja completamente contida na descrição exata da referência [18], é muito difícil utilizât-la na prắtica ou implementar aproximaçöes. Dada a importância de comprender melhor a dinâmica das correlaçôes quaanticas e do processo de decoerência em casos de interesse prátíco, preferimos aquí, neste trabalho, adiotar uma outra estratégia. Deduziremos então, no que segue a equação que seră o ponto de partida para as aproximaçöes posteriores.

A evoluçăo do operador densidade, $\rho(t)$, na representaçăo de interaçăo, conforme equaçăo 1.10 é dada por:

$$
i n \frac{d}{d t} p(t)=i \hbar \frac{d}{d t}\left(\mathrm{U}_{0}^{t}\left(t, t_{0}\right) \rho(t) \mathrm{U}_{0}\left(t, t_{0}\right)\right)
$$




$$
\begin{aligned}
& =-\mathrm{H}_{0} p(t)+\frac{\eta}{n} \mathrm{U}_{0}^{\dagger}\left(t, t_{0}\right)\left(\frac{a}{d t} p(t)\right) \mathrm{U}_{0}\left(t_{t} t_{0}\right)+\tilde{p}(t) \mathrm{H}_{0} \\
& =-\left[\mathrm{H}_{0}, \widetilde{p}(t)\right]+\mathrm{U}_{0}^{(t}\left(t_{3}, t_{0}\right)[\mathrm{H}, p(t)] \mathrm{U}_{0}\left(t, t_{0}\right) \\
& =-\left[\mathrm{H}_{0}, \tilde{\rho}(t)\right]+\mathrm{U}_{0}^{\dagger}\left(t, t_{0}\right)\left[\mathrm{H}_{0}+\mathrm{H}_{i \mathrm{int}}, \rho(t)\right] \mathrm{U}_{0}\left(t, t_{0}\right)
\end{aligned}
$$

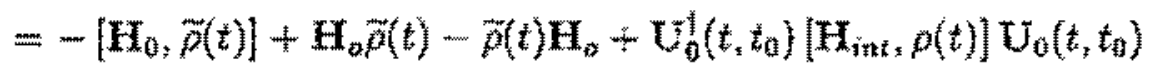

$$
\begin{aligned}
& =\mathrm{U}_{0}^{\dagger}\left(t, t_{0}\right) \mathrm{H}_{\mathrm{in} t} \rho \mathrm{U}_{0}\left(t, t_{0}\right)-\mathrm{U}_{0}^{\dagger}\left(t, t_{0}\right) p \mathrm{H}_{\text {int }} \mathrm{U}_{0}\left(t, t_{0}\right) \\
& =\overbrace{U_{0}^{\dagger}\left(t, t_{0}\right) \mathbf{H}_{i n t} U_{0}\left(t, t_{0}\right)}^{\vec{H}_{\text {nat }}(t)} \underbrace{U_{0}^{\dagger}\left(t, t_{0}\right) \rho \mathbf{U}_{0}\left(t, t_{0}\right)}_{\bar{\rho}(t)}- \\
& \ddot{p}(t) \\
& -\overbrace{U_{0}^{\dagger}\left(t, t_{0}\right) \rho U_{0}\left(t, t_{0}\right)} \underbrace{U_{0}^{f}\left(t, t_{0}\right) \mathbf{H}_{i n t} U_{0}\left(t, t_{0}\right)}_{\ddot{H}_{i n t}(t)}=\tilde{\mathbf{H}}_{i n t}(t) \tilde{\rho}(t)-\widetilde{\rho}(t) \tilde{\mathbf{H}}_{i n t}(t)
\end{aligned}
$$

ou seja,

$$
i \hbar \frac{d}{d t} \tilde{\rho}(t)=\left[\widetilde{\mathrm{H}}_{i n t}(t), \tilde{\rho}(t)\right] .
$$

A equação diferencial 1.20 é equivalente a equação integral

$$
\partial(t)=\tilde{\rho}(0)+\left(-\frac{i}{\hbar}\right) \int_{0}^{t} d t^{t}\left[\tilde{\mathrm{H}}_{\text {int }}\left(t^{t}\right), \tilde{\rho}\left(t^{t}\right)\right] .
$$

Nas ủltimas décadas, uma questão que tem assumido relevanncia crescente é o tratamento de sistemas quânticos abertos. A idéia central é que, apesar da Mecânica Quântica ser aplicada a sistemas fechados, na prática é impossivel isolar conpletamente o sistema do ambiente que o cerca. Para tornar o problema tratável, modularemos o anbiente como um barho de osciladores harmónícos e o acoplamento ao sistema de interesse, um oscilador harmönico, sera linear. Trataremos sistemas quânticos gerais cuja dinämica possa ser escrita por:

$$
\mathbf{H}=\mathbf{H}_{\mathcal{S}}+\mathbf{H}_{\mathfrak{R}}+\mathbf{H}_{\mathrm{in}}
$$

onde $\boldsymbol{H}_{S}$ é o hamiltoniano do sistema, $\mathrm{H}_{R}$ é o hamiltoniano do reserervatório (banho) e $\mathrm{H}_{\text {int }}$ é o hamiltoniano de interaçấo.

Os dois tipos mais utilizados de haxiltonianas que se encaixam nestas premissas ธล̃o: 
1. Posiçăomposição

$$
\begin{aligned}
& \mathbf{H}_{(C L)}=\underbrace{h w_{\mathbf{0}}\left(\mathrm{a}_{\mathrm{a}}+\frac{1}{2}\right)}_{\mathbf{H}_{s}}+\underbrace{h \sum_{k}\left(w_{k} \hat{\mathrm{c}}_{k}^{\dagger} \hat{\mathrm{c}}_{k}+\frac{1}{2}\right)}_{\mathbf{H}_{k}} \\
& +\underbrace{\sum_{k}\left(\hat{\mathbf{a}}+\hat{\mathbf{a}}^{\dagger}\right)\left(\gamma_{k} \hat{\mathbf{c}}_{k+}^{\dagger}+\gamma_{k}^{*} \hat{\mathbf{c}}_{k}\right)}_{\mathbf{H}_{n s}}
\end{aligned}
$$

2. Onda girante

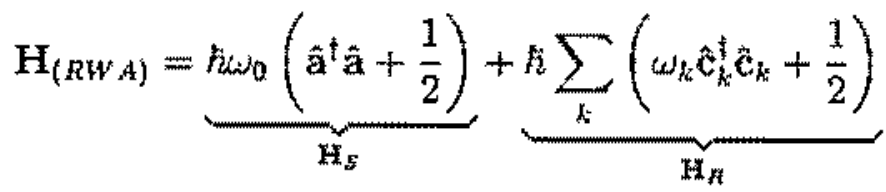

$$
\begin{aligned}
& +\underbrace{+\sum_{k} \gamma_{k} \hat{a}_{k}^{\dagger}+\gamma_{k}^{*} \hat{a}^{\dagger} \hat{c}_{k}}_{\mathbf{u}_{n t}}
\end{aligned}
$$

onde $\mathrm{H}_{(R W A)}$ decorre da primeira se desprezarmos termos não ressonantes. Isto é uma excelente aproximação no regime de acoplamento fraco $[20,21,19]$.

A densidade reduzida do sistema de interesse nestes dois casos pode ser obtida facilmente na aproximação de Born-Markov e a dedução dăs equações resultantes pode-ser encontrada em vắíos livros textos $[0,16,30,8]$ e nâo será repetida aquil. Oresultado e:

$$
\begin{aligned}
& i \frac{d}{d t} \rho_{S}(t)=L_{(C L)} \rho_{S}(t)=-\frac{i}{h}\left[H_{S}, p_{S}(t)\right] \\
& +k(\pi+1)\left(2 a \rho_{s}(t) \hat{a}^{\dagger}-\hat{a}^{\dagger} \hat{a} \rho_{S}(t)-\rho_{S}(t) \hat{a}^{\dagger} \hat{a}\right) \\
& +h \bar{n}\left(2 \hat{a}^{t} \rho_{S}(t) \hat{\mathbf{a}}-\hat{\mathbf{a}} \hat{a}^{\dagger} \rho_{S}(t)-\rho_{S}(t) \hat{a} \hat{a}^{i}\right) \\
& -k M\left(2 \hat{a}^{t} p \hat{a}^{1}-\left(\hat{a}^{\dagger}\right)^{2} p-p\left(\hat{a}^{t}\right)^{2}\right) \\
& -k M^{*}\left(2 \hat{a} \rho \mathbf{a}-\hat{a}^{2} \rho-\rho a^{2}\right), \\
& i \frac{d}{d t} \rho_{S}(t)=\mathcal{L}_{(\text {RWA })} \rho_{S}(t)=-\frac{i}{\hbar}\left[\mathbf{H}_{S}, \rho_{S}(t)\right]
\end{aligned}
$$

\footnotetext{
Inat segăo 4.1 faremos a deduçăo para dois osciladores
} 


$$
\begin{aligned}
& +k(\bar{n}+1)\left(2 \hat{a} p_{S}(t) \hat{a}^{\dagger}-\hat{a}^{\dagger} \hat{a} p_{S}(t)-p_{S}(t) \hat{a}^{\dagger} \hat{a}\right) \\
& +k \bar{n}\left(2 \hat{a}^{\dagger} p_{S}(t) \hat{a}-\hat{a} \hat{a}^{\dagger} p_{S}(t)-\rho_{S}(t) \hat{a} \hat{a}\right) .
\end{aligned}
$$

Se o banho for frio $\bar{n} \rightarrow 0$ podemos escrever ${ }^{2}$ :

$$
\frac{d}{d t} p_{S}(t)=-\frac{i}{\hbar}\left[H_{S}, p_{S}(t)\right]+k\left(2 \hat{a} p_{S}(t) \hat{a}^{t}-\hat{a}^{\dagger} \hat{a}_{S}(t)-p_{S}(t) \hat{a}^{\dagger} \hat{\mathbf{a}}\right)
$$

$O$ segundo termo nāo unitários na evoluçäo, caracterizados por anticomutadores e termos do tipo ât.â é em geral chamado, o dissipador.

\subsection{Decoerência, correlação e dissipação}

Um dos problemas mais intrigantes no que se refere a contrastes entre a Mecânica Clássica è a Mecânica Quãntica è a inexistência de superposiçöes coerentes de estados macroscópicamente distingüiveis na primeira. Em príncipio, a existência de fenômenos de interferência a nível microseópico implicaria no aparecimento do mesmo fenômeno a nivel macroscópico. O intrigante a a inexistência ciássica dos fenômenos de interferência quântica advindos de correlaçöes. A mais famosa ilustraçāo do problema foi proposta por Schrödinger em 1935 com o "Paradoxo do Gato" [22]. Na tentiva de resolver está questão, os conceitos de decoerência, de superposiçôes coerentes e dissipação aparecem como ingredientes fundamentais. Geralmente o sistema observado, que interage com o ambiente, relaxa en direção a um equilibrio térmico com o ambiente, fenômeno comunente conhecido como dissipação [12]. Por outro lado, o ambiente age sobre o sistema devido às flutuaçōes térmicas provocando a destruição de estados quânticos puros numa escala de tempo extremamente curta, processo esse que é denoninado decoerenncia (perda de pureza) $[9,13,10,3,6,7,2]$. Neste contexto, através desses processos, decoerêncías e díssipaçäo, num estado quântico composto por uma superposiçäo coerente de estados distingüiveis, torna-se uma mistura estatística. Apesar de ambos terem origen no acoplamento do sistema ao meio, dissipação e decoerència săo fenômenos distintos que podem ocorrer independentemente[3].

No presente trabalho trataremos especificamente destes tipos de efeitos nāo unitários.

Conforme mencionado anteriormente, embora os dois processo estejam relacionados intimamente, eles são essencialmente distintos. Um dos objetivos do presente trabalho é propor uma expansão pertubativa tanto para a decoerêteicia cono para a dissipacăa e estudar casos de intêresse. Para isso é conveniente introduzir uma mediw da de decoerência através do defeito de idempotência (ou entropia linear) conforme equaçẫo 1.14

$$
\delta_{i}(t)=1-\operatorname{Tr}_{i}^{2}
$$

\footnotetext{
"2 veja seçào 4.1
} 
e da dissipação

$$
\frac{E_{i}(t)}{E_{i}(0)}=\frac{\operatorname{tr}\left(\mathbf{H}_{i} \rho_{i}(t)\right)}{\operatorname{tr}\left(\mathbf{H}_{i} \rho_{i}(0)\right)}
$$

onde $i$ é um índice mudo, podendo representar o sistema, o ambiente ou a interaçāo e $E_{i}(t), E_{i}(0)$ são as energias do sistema " $i$ "no instante $t$ e no instante inicial respectivamente. 


\section{Capítulo 2}

\section{"Regra Aurea"para Decoerência e Dissipação}

No presente capítulo deduziremos expressôes gerais perturbativas para o cálculo do defeito de idempotência e da dissipaçāo. Aplicaçōes serào feitas para os hamiltonianos de un oscilador linearmente acoplado a um banho de osciladores, conforme equações 1.23 e 1.24. Estes exemplos ten caráter didático e nos mostrarão os mecanismos caracteristicos da díssipaçăo e decoèrencia. Finalmente, aplicaremos a teoria desenvolvida para estudar a decoerêticia e dissipação de uma superposiçăo coerente de estados coerentes. Mostraremos a relação quantitativa entre os tempos característicos dos dois processos.

\subsection{Expansão Perturbativa da Densidade Reduzi- da}

A dinâmica completa do sistema, equaçäo 1.22 , é descrita como mostrado no capítulo anterior pela equaçāo de Lioville-von Neumann, 1.17 ou na representaçāo de interaçäo 1.20.

A equaçăo ná representaçăo de interaçāo pode ser iterada e truncada numa dada ordem desde que a interação entre o sistema e o ambiente possa ser considerada fraca num sentido adequado.

A iteracăo é feita substituindo 1.21 en 1.20 atế segunda ordem em $\mathrm{H}_{\text {int }}$, obtendo:

$$
\begin{aligned}
& \tilde{\rho}(t)=\tilde{\rho}(0)+\frac{-i}{\hbar} \int_{0}^{t} d t^{\prime}\left[\widetilde{\mathrm{H}}_{i n t}\left(t^{t}\right), \tilde{\rho}\left(t^{\prime}\right)\right] \\
& =\widetilde{\rho}(0)-\frac{i}{\hbar} \int_{0}^{t} d t_{1}\left[\tilde{\mathrm{H}}_{i n i}\left(t_{1}\right), \tilde{\rho}(0)\right] \\
& -\frac{1}{2 \hbar} \int_{0}^{t} d t_{2} \int_{0}^{t_{2}} d t_{1}\left[\tilde{\mathrm{H}}_{i s t}\left(t_{2}\right),\left[\tilde{\mathrm{H}}_{i n t}\left(t_{1}\right), \tilde{p}(0)\right]\right]+\mathbb{O}\left(\mathrm{H}_{\text {int }}^{3}\right) \\
& =\gamma^{(0)}+p^{(1)}+p^{(2)}+\cdots
\end{aligned}
$$


A dinâmica reduzida do sistema de interesse $S$ ou $R$ será dada por

$$
\tilde{p}_{i}=T r_{j} \ddot{p} \quad \text { onde } i, j \text { representam os sistemas e } i \not j \text {. }
$$

Esta é a equaçäo de partida para o cálculo pertubativo do defeito de idempotência, 1.28, e dissipaçäo, 1.29, definidos no capitulo anterior. Dentro do contexto perturbativo exposto e supondo que a condiçăo inicial $\widetilde{\rho}(0)$ é fatorizada, podemos escrever expressōes gerais para $\hat{\delta}_{S}(\hat{t})$ e $E_{5}(t)$ ou para $\hat{o}_{R}(t)$ e $E_{R}(t)$ como segue:

$$
\begin{aligned}
& \delta_{j}^{(1)}(t)=0 \\
& f_{j}^{(2)}(t)=-t r\left(\rho_{j}^{(1)}(t)\right)^{2}-2 t r \rho_{j}^{(0)}(t) \rho_{j}^{(2)}(t) \\
& E_{j}^{(1)}(t)=\operatorname{Tr} \mathrm{H}_{j} \rho_{j}^{(1)}(t) \\
& E_{j}^{(2)}(t)=\operatorname{Tr} \mathrm{H}_{j} \rho_{j}^{(2)}(t) \quad j=S \text { ou } R .
\end{aligned}
$$

\subsection{Acoplamento Linear a um banho de Oscilado- res}

Consideremos inicialmente a dinámíca dada pela hamiltoniana $\mathrm{H}_{R W_{A}}$ da equaçầo, 1.24 , com $\gamma_{k}$ real por simplicidade.

Para uma condiçäo inicial, $\rho(0)$, fatorizável

$$
\rho(0)=\rho_{S}(0) \otimes \rho_{R}(0)
$$

podemos escrever a dinâníca reduzida numa forma compacta por:

$$
\begin{aligned}
& \tilde{\rho}^{1 !}(t)=-\hat{T} r_{m} \int_{0}^{t} d t_{1}\left[\tilde{\mathrm{H}}_{\mathrm{in}}\left(t_{1}\right), \tilde{\rho}(0)\right]
\end{aligned}
$$

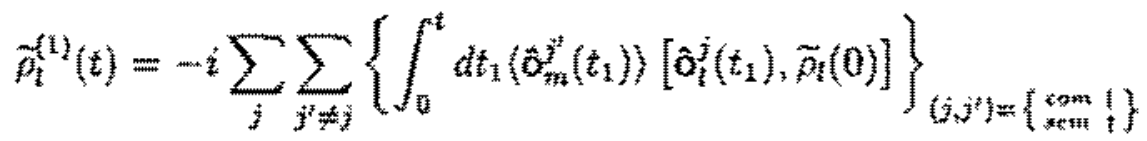

$$
\begin{aligned}
& \tilde{\rho}_{1}^{(2)}(t)=-\frac{1}{2} T r_{m} \int_{0}^{t} d t_{2} \int_{0}^{t} d t_{1}\left[\tilde{\mathbf{H}}_{i n t}\left(t_{2}\right),\left[\tilde{\mathbf{H}}_{i n t}\left(t_{1}\right), \tilde{p}(0)\right]\right] \\
& \hat{p}_{l}^{(2)}(t)=-\frac{1}{2} \sum_{j} \sum_{j \neq j}\left\{\int_{0}^{t} d t_{2} \int_{0}^{t_{2}} d t_{1}\left[\hat{o}_{l}^{j}\left(t_{2}\right),\left[\hat{o}_{l}^{j}\left(t_{1}\right), \tilde{p}_{l}(0)\right]\right]\left\langle\hat{o}_{m}^{j^{\prime}}\left(t_{2}\right) \hat{o}_{m}^{j^{t}}\left(t_{1}\right)\right\rangle\right. \\
& +\left[\hat{o}_{l}^{j}\left(t_{2}\right), \hat{o}_{l}^{j^{t}}\left(t_{1}\right) \hat{\rho}_{i}(0)\right]\left(\hat{o}_{m}^{j^{\prime}}\left(t_{2}\right) \hat{\mathrm{o}}_{m}^{j}\left(t_{1}\right)\right\rangle
\end{aligned}
$$

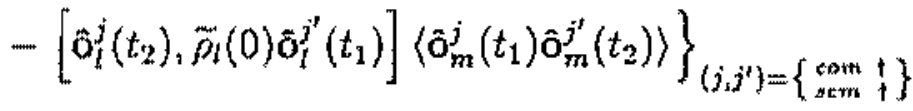

onde: 
1. Se "le for o sistema " $m$ " for o reservatório entăo

$$
\begin{aligned}
& \hat{o}_{m}^{j}(t)= \begin{cases}\Gamma^{\dagger}(t)=\sum_{k} \gamma_{k} e^{i k\left(\omega_{k}-\omega_{0}\right) t} c_{k}^{t} & \text { para } j=\operatorname{com} \dagger \\
\Gamma(t)=\sum_{k} \gamma_{k} e^{-i \hbar\left(w_{k}-\omega_{0}\right) t} c_{k} & \text { para } j=\operatorname{sem} \dagger\end{cases} \\
& \hat{o}_{(}^{j}(t)= \begin{cases}\hat{a}^{\dagger} & \text { para } j=\operatorname{com} \dagger \\
\hat{a} & \text { para } j=\operatorname{sem} \dagger .\end{cases}
\end{aligned}
$$

Logo

$$
\begin{aligned}
& \rho_{S}^{(1)}(t)=-\int_{0}^{t} d t_{1}\left([ a ^ { \dagger } , \tilde { p } _ { S } ( 0 ) ] \left\langle\Gamma\left(t_{1}\right)+\left[\hat{a}_{s}, \tilde{p}_{s}(0)\right]\left(\Gamma\left(t_{1}\right)\right)\right.\right. \\
& \rho_{S}^{(2)}(t)=-\frac{1}{2} \int_{0}^{t} \int_{0}^{t_{2}} d t_{2} d t_{1}\left\{\left[\hat{a},\left[\hat{a}, \tilde{p}_{S}(0)\right] \mid\left\langle\Gamma^{t}\left(t_{2}\right) \Gamma^{\Gamma t}\left(t_{1}\right)\right\}\right.\right. \\
& +\left[\hat{\mathbf{a}}^{\dagger}:\left[\hat{\mathbf{a}}^{\dagger}, \hat{p}_{S}(0)\right]\right]\left\langle\Gamma\left(\hat{t}_{2}\right) \Gamma\left(t_{1}\right)\right\rangle \\
& \div\left[\hat{a}^{\dagger}, \hat{a} \vec{\rho} s(0)\right]\left\langle\Gamma\left(t_{2}\right) \Gamma^{\dagger}\left(t_{1}\right)\right\rangle+\left[\hat{a}_{4} \hat{a}^{\dagger} \ddot{p}_{S}(0)\right]\left\langle\Gamma^{\dagger}\left(t_{2}\right) \Gamma\left(t_{1}\right)\right\rangle \\
& \left.-\left[\hat{a}, \tilde{\rho}_{S}(0) \hat{a}\right]\left\langle\Gamma^{\prime}\left(t_{1}\right) \Gamma^{\dagger}\left(t_{1}\right)\right\rangle-\left[\hat{a}^{\dagger}, \tilde{\rho}_{S}(0) \hat{a}\right]\left\langle\Gamma^{\dagger}\left(t_{1}\right) \Gamma\left(t_{2}\right)\right\rangle\right] \text {. }
\end{aligned}
$$

2. Caso contrário, se "l" for o reservatónio e " $m$ " for o sistema entä́o teremos:

$$
\begin{aligned}
\tilde{\rho}_{R}^{(1)}(t) & =-\int_{0}^{t} d t_{1}\left\{\left[\Gamma^{\dagger}\left(t_{1}\right), \tilde{\rho}_{R}(0)\right]\langle\hat{a}\rangle+\left[\Gamma\left(t_{1}\right), \tilde{\rho}_{R}(0)\right]\left\langle\hat{a}^{\dagger}\right\rangle\right\} \\
\tilde{\rho}_{R}^{(2)}(t) & =-\frac{1}{2} \int_{0}^{t} \int_{0}^{t_{2}} d t_{2} d t_{1}\left\{\Gamma\left(t_{2}\right),\left[\Gamma\left(t_{1}\right), \tilde{\rho}_{R}(0)\right]\right]\left\langle\hat{a}^{\dagger 2}\right\rangle \\
& +\left[\Gamma^{\dagger}\left(t_{2}\right),\left[\Gamma^{\dagger}\left(t_{1}\right), \rho(0)\right]\right]\left\langle\hat{a}^{2}\right\rangle \\
& +\left[\Gamma^{\dagger}\left(t_{2}\right), \Gamma\left(t_{1}\right) \tilde{\rho}_{R}(0)\right]\left\langle\hat{\mathbf{a}}^{\dagger}\right\rangle+\left[\Gamma\left(t_{2}\right), \Gamma^{\dagger}\left(t_{1}\right) \rho(0)\right]\left\langle\hat{a}^{\dagger} \hat{a}\right\rangle \\
& \left.-\left[\Gamma\left(t_{2}\right), \tilde{p}_{R}(0) \Gamma^{\dagger}\left(t_{1}\right)\right]\left\langle\hat{a} \hat{a}^{\dagger}\right\rangle-\left[\Gamma^{\dagger}\left(t_{2}\right), \tilde{\rho}_{R}(0) \Gamma\left(t_{1}\right)\right]\left\langle\hat{a}^{\dagger} \vec{a}\right\rangle\right\}
\end{aligned}
$$

Tomemos a condiçāo inicial

$$
\begin{aligned}
& \rho_{S}(0)=|n\rangle\langle n|, \quad p_{n}(0)=\frac{1}{2} e^{-\beta K_{n}}, \\
& p(0)=\mid n)|n| \otimes \frac{1}{2} e^{-B \mathrm{H}_{n}},
\end{aligned}
$$

onde $|n\rangle\langle n|$ é a densidade correspondente a um auto estado do oscilador principal, $\mathrm{H}_{R}$ é dado pela equaçāo 1.24 \& $Z$ a funçäo de partiçăo correspondente. Com isto temos todos os ingredientes necessários para o callculo perturbativo da decoerễncia e dissipação. A dinämica reduzida para o subsistema $S$, em sẹgunda ordem, será:

$$
\begin{aligned}
& \left.\rho_{\mathcal{S}}^{(2)}(t)=\mid n\right)\left(n \mid-\sum_{k} \gamma^{2} \frac{\sin ^{2} \frac{k_{k}}{2}}{\left(\frac{b_{2}}{2}\right)^{2}}\left\{\left((2 n+1) \bar{n}_{k}+n|| n\right)(n \mid\right.\right. \\
& \left.\left.-n\left(\bar{n}_{k}+1\right) \mid n-1\right)\left(n-1\left|-(n-1) n_{n}\right| n+1\right)(n+1)\right\}
\end{aligned}
$$


onde $\delta_{k}=\left(\omega_{\phi}-\omega_{k}\right)$ e $\bar{n}_{k}$ é o número da excitaçöes do banhol.

Neste caso simples já podemos identificar alguns dos mecanismos importantes da dinămica reduzida a tempos curtos: primeiramente notamos que $\rho_{S}^{(2)}(t)$ deixará de ser um estado puro adquirindo as contribuiçôes dos termos diagonais $|n-1\rangle\langle n-1| \mathrm{e}$ $|n+1\rangle\langle n+1|$. Alëm disto, podemos claramente identificar o que acontece a tempos muito curtos, no que é freqüentemente chamado regime prë-cinético: para tempos tais que

$$
\delta_{k} t<1, \quad \text { otermo dependente do tempo } \frac{\sin ^{2} \frac{k_{k}}{2} t}{\left(\frac{s_{k}}{2}\right)^{2}} \rightarrow t^{2}
$$

e portanto a expressäo para a densidade redurida fica independente das frequências do problema. Em particular isto implica que todos os modos do banho para os quais $\gamma_{k} \neq 0$ văo contribuir para a densidade reduzida do sistema de interesse, mesmo aqueles com energias nuito diferentes da energia inicial disponivel. Vemos então que o regime pró̀-cinético é dominado pelo princípio da incerteza energia-tempo. Após este tempo, ie $\delta_{k} t \sim 1$, a função $\left(\sin ^{2} \frac{\alpha_{s}}{2}\right)\left(\frac{\delta_{b}}{2}\right)^{-2}$ começa a limitar a contribuiçẫo dos estados mats distantes tal que

$$
\frac{\sin ^{2} \frac{b_{k} t}{2}}{\left(\frac{h_{k}}{2}\right)^{2}} \rightarrow \delta\left(\omega_{q}-\omega_{k}\right) t
$$

e a contribuiçäo do reser vatório à evoluçäo do subsistema de interesse será predominantemente através dos estados com energia próximas a $w_{0}$. Inicia-se então o regime cinético, onde se aplicarn as consideraçōes que serảo feitas na dedução da equaçào mestra

Substituindo $\rho_{S}^{(2)}(t)$ nas equaçöes 2.1 e.2.2 encontramos respectivamente:

$$
\delta_{S}(t)=\delta_{S}^{(2)}(t)=2 \sum_{k} \gamma_{k}^{2}\left\{(2 n+1) \vec{n}_{k}+n\right\} \frac{\sin ^{2} \frac{\delta}{k}_{k} t}{\left(\frac{\delta_{k}}{2}\right)^{2}}
$$

e

$$
\begin{aligned}
E_{\left.S_{(R W A}\right)}^{(2)}(t) & =\hbar \omega_{0} \sum_{k} \gamma_{k}^{2}\left(\bar{n}_{k}-n\right) \frac{\sin ^{2} \frac{\delta_{k}}{2} t}{\left(\frac{\delta_{k}}{2}\right)^{2}} \\
& \downarrow \\
E_{S_{(R W A)}}(t) & =n h \omega_{0}+\hbar \omega_{0} \sum_{k} \gamma_{k}^{2}\left(\bar{n}_{k}-n\right) \frac{\sin ^{2} \frac{\delta_{k}}{2} t}{\left(\frac{\delta_{k}}{2}\right)^{2}}
\end{aligned}
$$

Das expressöes acima vê-se imediatamente algumas diferenças essenciais entre os processos de decoerência e dissipaçäo: no caso da decoerència, toclas as contribuições incorporadas a $\rho_{S}^{(2)}(t)$ pela interaçầo adicionam-se contribuindo positivamente para

\footnotetext{
${ }^{1}$ matores detalhes na seçäo 4.1

"seçăo 4.1
} 
a perda de pureza. No caso da dissipaçäo, vemos que os modos que tem $\bar{n}_{k}>n$ tendem a excitar o sistema enquanto que os que tem $\pi_{k}<n$ tendem a desexciti lo. O resultado portanto un balanço entre perda ganho de energia como se vê claramente na expressäo 2.4. Em particular este balanço poderá ser nulonão havendo dissipaçäo mas havendo decoerência. É razónel entäo pensar que o tempo característico da perda de pureza é mais rápido do que da perda de energia. Isto será demonstrado mais rigorosamente na secçäo2.3. É possivel tambem usar um estado inicial do tipo

$$
\rho(0)=p_{S}^{(0)} Q^{\frac{e^{-\beta \Psi_{n}}}{2}}
$$

onde $p_{3}(0)$ um estado inicial qualquer do sistema de interesse. Neste caso obtemos para a dissipaça e decoerencia at segunda ordem

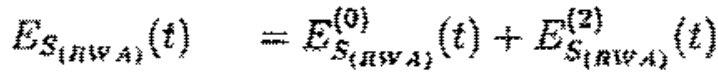

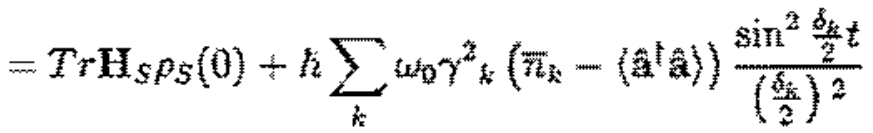

$$
\begin{aligned}
& E_{R_{(R W A)}}(t)=E_{R_{(A W A)}}^{(0)}(t)+E_{R_{\left(R W_{A}\right)}^{(2)}}^{(t)} \\
& =\operatorname{rr} \mathrm{H}_{R} \rho_{R}(0)-\hbar \sum_{k} \omega_{k} \gamma_{k}^{2}\left(\bar{n}_{k}-\langle a \hat{a} \hat{a}\rangle\right) \frac{\sin ^{2} \frac{\delta_{k}}{2} t}{\left(\frac{\delta_{k}}{2}\right)^{2}} \\
& \delta_{s_{(k n, A)}}(t)=1-\operatorname{tr} \rho_{S}^{2}(0)
\end{aligned}
$$

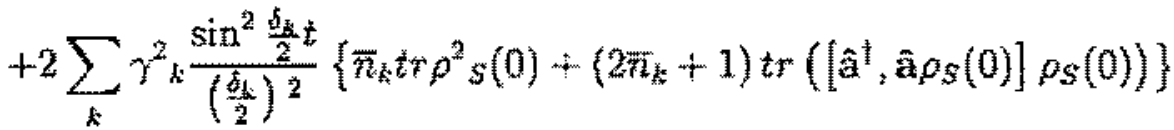

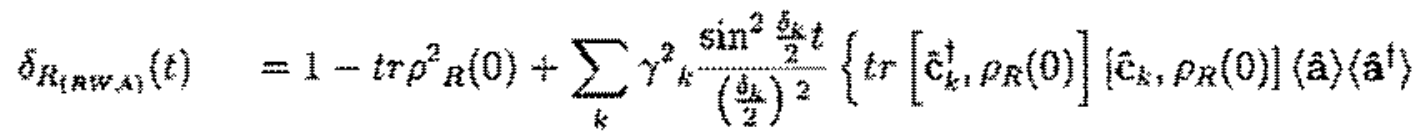

$$
\begin{aligned}
& \left.+2\left\langle\hat{a}^{\dagger} \hat{a}\right) \operatorname{tr} \rho_{R}^{2}(0)+2(2(a+a)+1) t r\left(\left[\hat{\mathrm{c}}_{k}^{\dagger} \hat{\mathrm{c}}_{\mathrm{k}} \rho_{n}(0)\right] \rho_{R}(0)\right)\right\} \text {. }
\end{aligned}
$$

E claro que o resultado anterior pode set imediatamente obtido das expressōes acima. 0 fato da energia total se conservar,

$$
\Delta\left\{E_{S_{(A W A)}}(t)+E_{R_{(R W A)}}(t)+E_{i n t_{(R W A)}}(t)\right\}=0
$$

nos leva a conchir em segunda ordem, usando a condiçào 2.3 , que a correçă à interacắo se anula, ou seja,

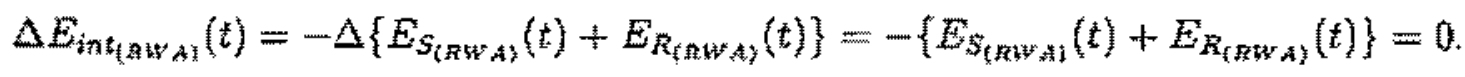


Por outro lado, os dois subsistemas perdem pureza cono pode ser visto a partir de $\delta_{S}(t)$ e $\delta_{R}(t)$ como conseqüência direta da interaçäo pois $\delta_{S}(t)$ e $\delta_{R}(t)$ tomam-se positivos.

Um caso interessante e 0 acoplamento posição posição dado pela equaçāo 1.23 onde consideraremos novamente $\gamma_{k}$ real por simplicidade. Tonando a mesma condiçăo inicial 2.5 e usando as nesmas técnicas é simples obter as expressôes para $E^{(2)}(t)$ e $d^{(2)}(t)$. No entanto estas expressôt säo pouco elucidativas e por isso nâa as escreveremos explicitamente. Vale a pena, entretanto investigar um fenôneno que acontece com a variaçăo de energia na interaçăo. Em segunda ordem,

$$
E_{\text {in }}^{(2)}(t)=-E_{\text {(2) }}^{(2)}(t)-E_{s}^{(2)}(t)
$$

assegurando a conservaçäo da energia, como deve ser. Para fazer este cálculo utilizaremos uma expansăo en série de Tyylor no tempo de ${ }^{\prime}\left(\boldsymbol{H}_{\text {int }} p_{\text {int }}^{(2)}(t)\right)$ dos subsistemas a tempos curtos. Mostramos entäo apenas as expressöes em ordem de $t^{2}$ para as energias do sistema de interesse, do reservatóno e do acoplamento

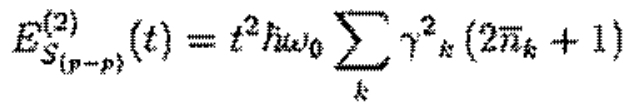

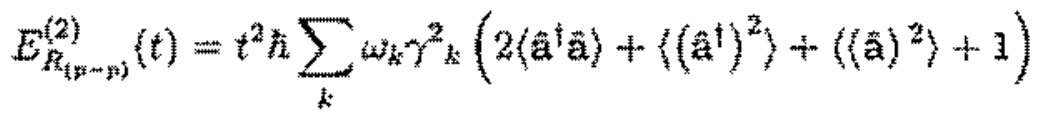

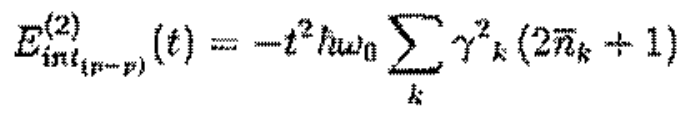

$$
\begin{aligned}
& -t^{2} n \sum_{k j} w_{k} \gamma_{k}^{2}\left(2\left\langle\hat{\mathbf{a}}^{\dagger} \hat{\mathbf{a}}\right\rangle+\left\langle\left(\hat{\mathbf{a}}^{\dagger}\right)^{2}\right\rangle+\left\langle\langle\hat{\mathbf{a}})^{2}\right\rangle+1\right)
\end{aligned}
$$

Note que aqui, contrariamente ao que ocorre no caso anterior, as duas energias crescem. Em particular, a energia do oscilador principal cresce independentemente do estado incial $\rho(0)$. Isto quer dizer que mestno que o estado inicial seja o estado fundanental do oscilador em questäo, e mesmo se os osciladores do banho também estiverem em seus estados fundamentais, $\bar{n}_{k}=0$, essa energia aumenta. Isto é um efeito quântico devido à interação. No caso anterior, o estado fundamental do hamiltoniano de partida consiste en ter todos os osciladores individualmente em seu estados fundamentais. Aqui isto já nâa é mais verdade e existe um estado com energia menox do que a deste, que é o estado fundamental do sistema fora da RWA. Entäo é possivel, para o estado inicialmente utilizado, uma tentativa de atingir esse estado de menor eneryia, sendo para isso necessário ceder energia.

\subsection{Decoerência e Dissipação de uma Superpo- sição Coerente}

Vamos agora aplicar o esquema perturbativo desenvolvido para estudar quantitativamente a relação entre as escalas de tempo características da decoerência e dissipação para un estado inicial especial, uma superposiçăo coerente de estados coerentes. 
Seja

$$
\rho(0)=N^{2}(\alpha)(|\alpha\rangle+|-\alpha\rangle)\left(\left\langle\alpha|+\langle-\alpha|) \otimes \prod_{k} \mid \hat{\mathbf{o}}_{k}\right\rangle\left\langle\hat{\mathbf{o}}_{k}\right|\right.
$$

com

$$
N(\alpha)=\left(2+2 e^{-2|\alpha|^{2}}\right)^{-\frac{1}{3}}
$$

que evolui sob a hamiltoniana $\mathbf{H}_{R W A}$ equação 1.24. Note que no estado inicial considerado o banho encontra-se a temperatura nula $\overline{n_{k}} \longrightarrow 0$. O símbolo $\left|\hat{o}_{k}\right\rangle$ indica que o k-ésimo oscilador encontra-se no estado fundamental. Obtemos até segunda ordem conforme equaçāo 2.6

$$
E_{S_{(R W A)}}(t)=E_{S_{(R W A)}^{(0)}}^{(t)}-N^{2}(\alpha) \hbar \omega_{0}|\alpha|^{2}\left(1-e^{-2|\alpha|^{2}}\right) \sum_{k} \gamma_{k}^{2} \frac{\sin ^{2} \frac{\delta_{k}}{2} t}{\left(\frac{\delta_{k}}{2}\right)^{2}}
$$

para a energia (dissipação). Note que o sistema de interesse vai perder energia para o banho, neste caso. Por outro lado, da equação 2.8

$$
\delta_{S_{(R W A)}}(t)=2 N^{2}(\alpha)|\alpha|^{2}\left(1-e^{-2|\alpha|^{2}}\right) \sum_{k} \gamma_{k}^{2} \frac{\sin ^{2} \frac{\delta_{k}}{2} t}{\left(\frac{\delta_{k}}{2}\right)^{2}} .
$$

Para definir tempos característicos de decoerência e dissipação fazemos o seguinte: supomos um espectro suave e contínuo para o reservatório e uma dependência suave de $\gamma_{k}$ com $k$. Nestas condiçōes, as somas que aparecem nas expressōes acima podem ser convertidas em integrais. Usando uma densidade de $\operatorname{estados}^{3} D(\omega)$ e a condição 2.3 , escrevemos

$$
\begin{aligned}
& E_{\left.S_{\langle R W A}\right)}(t)=E_{S_{(R W A)}^{(0)}}(t)-N^{2}(\alpha) \hbar \omega_{0}|\alpha|^{2}\left(1-e^{-2|\alpha|^{2}}\right) \gamma^{2}\left(\omega_{0}\right) D\left(\omega_{0}\right) t \\
& \delta_{S_{(R W A}}(t)=2 N^{2}(\alpha)|\alpha|^{2}\left(1-e^{-2|\alpha|^{2}}\right) \gamma^{2}\left(\omega_{0}\right) D\left(\omega_{0}\right) t
\end{aligned}
$$

o que nos permite definir as escalas de tempo da seguinte forma

$$
-\frac{E_{S_{(R W A)}^{(0)}(t)-E_{S_{(R W A)}}(t)}}{E_{S_{(R W A)}^{(0) p u r o}}^{(\text {W }}}=\frac{t}{\tau_{\text {diss }}}
$$

onde

$$
E_{S_{(R W A)}^{(0) p u r o}}^{(0)}=\left\langle\alpha\left|\hbar \omega_{0}\left(\hat{\mathrm{a}}^{\dagger} \hat{\mathrm{a}}+\frac{1}{2}\right)\right| \alpha\right\rangle=\hbar \omega_{0}\left(|\alpha|^{2}+\frac{1}{2}\right)
$$

Assim,

$$
\tau_{d i s s}^{-1}=N^{2}(\alpha) \frac{|\alpha|^{2}\left(1-e^{-2|\alpha|^{2}}\right) \gamma^{2}\left(\omega_{0}\right) D\left(\omega_{0}\right)}{|\alpha|^{2}+\frac{1}{2}} .
$$

\footnotetext{
${ }^{3}$ maiores detalhes na seçāo 4.1
} 
Analogamente definimos

$$
\delta_{S_{(R W A)}}(t)=\frac{t}{\tau_{d e c}}
$$

com

$$
\tau_{d e c}^{-1}=2 N^{2}(\alpha)|\alpha|^{2}\left(1-e^{-2|\alpha|^{2}}\right) \gamma^{2}\left(\omega_{0}\right) D\left(\omega_{0}\right)
$$

A relação entre esses tempos é

$$
\frac{\tau_{\text {diss }}}{\tau_{\text {dec }}}=2|\alpha|^{2}+1
$$

Esta relação foi obtida na referência [7] de forma qualitativa. Mostramos então que o tempo de decoerência é de fato mais curto que o tempo de dissipaçāo e será tanto menor quanto maior for $|\alpha|^{2}$, ie, quanto mais macroscópico for o estado de "gato de Schrödinger ". Esta relação espelha matematicamente o entendimento da emergência do mundo clássico: quanto mais "clássico"maior o número médio de fotons dos estados que compōe a superposição coerente, maior $|\alpha|^{2}$, mais difícil será detectar efeitos quânticos que eles passam a produzir, pois a coerência entre as componentes será perdida rapidamente. 


\section{Capítulo 3}

\section{Técnicas de Lie para Superoperadores}

No presente capitulo mostramos como a algebra de Lie para superope. radores pode ser utilizada para o desacoplamentode uma álgebra que envolve dois graus de liberdade. Particular ênfase é dada ao cálculo do operador densidade. Discutimos os vários métodos possíveis ilustrados como exemplos.

\section{1 Álgebra de Lie para Superoperadores}

A soluçäo de equação de Liouville-von Neumann, 4.16, quando o Liuvilliano, $\mathcal{L}$, é independente do tempo é dada por

$$
\rho(t)=e^{t t} \rho_{0}
$$

Em muitos casos de interesse $\mathcal{L}$ é uma combinaçăo linear de um conjunto de superoperadores que queremos expressar como um produto ordenado de exponenciais destes superoperadores.

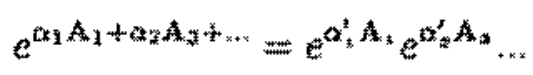

onde o conjunto dos elementos $\left\{A_{i}\right\}$ formam uma algebra de Lie $[28]$ e $\left\{\alpha_{i}\right\}$ sẫo números complexos.

Os superoperadores atuam no espaço formado pelos operadores lineares que atuan no espaço unitário $\varepsilon$. $O$ espaço de atuaçăo dos superoperadores e chamado espaço de Litountle $\mathrm{A}$.

Uma algebra de Lie é definida por um conjunto de operadores $\mathcal{A}=\left\{A_{1}, A_{2}, \ldots, A_{n}\right\}$ fechado sob comutaçăo, ou seja, a operaçäo de comutaçäo entre qualquer par de elementos do conjunto, resulta numa combinaçấo linear de elementos do próprio 
conjunto.

$$
\left[A_{i}, A_{j}\right]=\sum_{k=1}^{n} c_{i j}^{(k)} A_{k}
$$

onde o número de elementos da algebra, $n$, define sua dimensionalidade $e_{i, j}^{(k)}$ são denominadas constantes de estrutura da atgebra que satisfaz a identidade de Jacobi

$$
\sum_{l, m}\left(c_{i, j}^{(n)} c_{i, k}^{(m)}+c_{j, k}^{(n)} c_{l, i}^{(m)}+c_{k, 3}^{(n)} c_{l, j}^{(n)}\right)=0
$$

a anti simetria do comutador

$$
c_{i, j}^{(k)}=-c_{j, i}^{(k)}
$$

Reciprocamente, dado as constantes $c_{i, j}^{(k)}$ que satisfaçam estas igualdades, elas serão as constantes de uma algebra de lite.

A principal vantagem do uso das técnicas algetbricas de Lie reside na sua generalidade. Pois, uma vez que uma álgetra é gerada por um espaço vetorial, uma base pode ser escolhida para o espaço vetorial de qualquer álgebra.

No caso específico que envolve osciladores harmônicos surge o aparecimento de superoperadores bosônicos que para un hamiltoniano de interaçăo linear nas variáves $\hat{x}$ e $\hat{p}$ dos osciladores, estes superoperadores bosónicos formam uma álgebra fechada, o que permite aplicar as técnicas da algebra de Lie.

Os superoperadores bosonicos representam a açäo dos superoperadores, de criaçäo e aniquilaçäo do oscilador harmônico, ầ e âa respectivamente, sobre um operador A qualquer,

$$
\left(\hat{a}_{1}\right) A=\hat{a}_{1} A \quad\left(\hat{a}_{1}^{\dagger}\right) A=\hat{a}_{1}^{\dagger} A \quad\left(\hat{a}_{1}\right) A=A \hat{a}_{1} \quad\left(\cdot \hat{a}_{1}^{\dagger}\right) A=A a_{1}^{\dagger}
$$

que obedecen as relaçöes de comutaçăo

$$
\left[\left(\hat{a}_{1}\right),\left(\hat{a}_{1}^{t}\right)\right]=(1 \cdot)=1, \quad\left[\left(\hat{\mathbf{a}}_{1}\right),\left(-\hat{a}_{1}^{\dagger}\right)\right]=-(\cdot 1)=-1
$$

sendo nula as demais combinaçöes de comutaçāo.

O produto bilinear dos superoperadores bosónicos é dado por:

$$
\begin{aligned}
& \left(\hat{a}_{1}{ }^{2}\right)=\left(\hat{a}_{1}\right)^{2}=\left(a_{1}\right)\left(\hat{a}_{1}\right) \\
& \left(\cdot \hat{a}_{1}^{2}\right)=\left(\cdot \vec{a}_{1}\right)^{2}=\left(\cdot \vec{a}_{1}\right)\left(\cdot \hat{a}_{1}\right)
\end{aligned}
$$

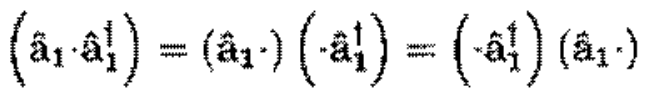

$$
\begin{aligned}
& \left(\hat{a}_{1} \vec{a}_{1} \cdot\right)=\left(\hat{a}_{1}^{\dagger}\right)\left(\hat{a}_{1} \cdot\right) \\
& \left(a_{1} a_{2}\right)^{2}=\left(a_{t}^{+}\right)^{2}\left(a_{2} \cdot\right)^{2} \Leftrightarrow\left[a_{1:}^{+} a_{2}\right]=0 \\
& \left.\left(\hat{a}_{1}^{t} a_{1}\right)^{2}=\left(a_{1}^{\dagger} a_{2} \cdot\right)\left(a_{1}^{a} a_{1} \cdot\right)\right)
\end{aligned}
$$

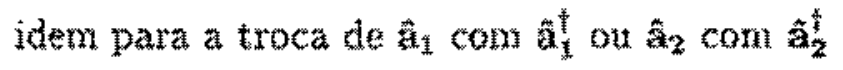




\subsubsection{Transformação de Similaridade e Fórmula de Haus- dorff}

Sejam A a B operadores pertencentes a algebra de Lie, e $x$ um número complexo.

Seja $f(x)=e^{x A} \mathrm{~B} e^{-2 \mathrm{~A}}$ sujeito à condiçăo $f(x=0)=\mathrm{B}$,

entāo, $f(x)$ satisfaz a equaçào diferencial de primeira ordem

$$
\frac{d}{d x} f(x)=\mathrm{A} e^{ \pm \mathrm{A}} \mathrm{Be}-\mathrm{A}+e^{\mathrm{AA}} \mathrm{B} e^{-\mathrm{AA}}(-\mathrm{A})=\mathrm{A} f(x)-f(x) \mathrm{A}=[\mathrm{A}, f(x)]
$$

cuja solução seră:

$$
f(x)=\mathrm{B}+\int_{0}^{x} d x^{2}\left[\mathrm{~A}, f\left(x^{t}\right)\right]
$$

Substituindo $f\left(x^{\prime}\right)=\mathbf{B}+\int_{0}^{x^{\prime}} d x^{\prime \prime}\left[\mathbf{A}, f\left(x^{\prime \prime}\right)\right]$ e prosseguindo iterativamente, obtemos:

$f(x)=e^{x \mathbf{A}} \mathbf{B} e^{-x \hat{A}}=\mathbf{B}+x[\mathbf{A}, \mathbf{B}]+\frac{x^{2}}{2 !}[\mathbf{A},[\mathbf{A}, \mathbf{B}]+\ldots+\frac{x^{n}}{n !} \overbrace{[\mathbf{A},[\mathbf{n}, \ldots[\mathbf{A}, \mathbf{B}] \ldots]}$

Podemos ainda escrever a equaçăo diferencial para $f(x)$ na forma:

$\frac{d}{d x} f(x)=[\mathrm{A}, \cdot] f(x)$

cuja solução serâ:

$f(x)=e^{x[\mathbf{A},]} f(x=0)=e^{x[\mathbf{A},} \cdot \mathbf{B}$

obtendo assim a identidade:

$e^{x \boldsymbol{A}} \mathrm{B} e^{-x \cdot \mathrm{A}}=e^{x[\mathrm{~A}, / \mathrm{B}}$

Resumidamente, uma transformaçăo de sinvilaridade de Hausdorff $[24,36]$ é definida por:

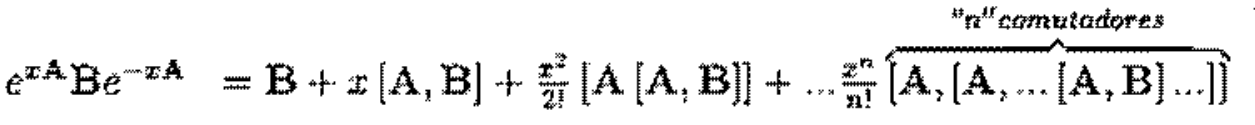

$$
\begin{aligned}
& =\sum_{n=0}^{\infty} \frac{z^{n}}{n !}\left[A \cdot J^{n} \mathbf{B}\right. \\
& =e^{x \mid \mathrm{A}} \cdot \mathrm{B}
\end{aligned}
$$


onde, $\mathrm{A}$ e $\mathrm{B} \in \mathcal{A}$ e o superoperador $[\mathbf{A},]^{n}$ indica:

$$
\begin{aligned}
& {[A, \cdot]^{D} B=B} \\
& {[A, \cdot B=[A, B]} \\
& {[A,]^{2} B=[A, \cdot][A, \cdot B)=[A, \cdot]([A, B])=[A,[A, B]]} \\
& {[A,]^{n} B=[A,]^{n-1}[A, B]}
\end{aligned}
$$

Atraves da definięăo da equaçāo 3.5 pode-se mostrar que se expandirnos em série

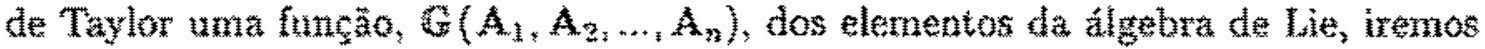
obter:

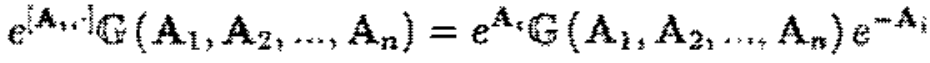

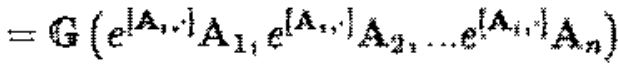

\subsubsection{Fórmulas de Baker-Campbell-Hausdorff e de Zasse- nhaus}

A fórmula de Baker-Campbell-Hausdorff (fórmula BCH) estabelece que para quaisquer operador $\mathrm{A}$ e $\mathrm{B} \in \mathcal{A}$ existe um operador $\mathrm{C} \in \mathcal{A}$ tal que:

$$
e^{\mathrm{A}} e^{\mathrm{B}}=e^{\mathrm{C}}
$$

onde $[23]$

$$
\mathrm{C}=\mathrm{A}+\mathrm{Z}_{1}+\mathrm{Z}_{2}+\ldots+\mathrm{Z}_{\mathrm{n}}+\ldots
$$

com

$$
\begin{aligned}
& \mathrm{Z}_{1}=\mathrm{B} \\
& \mathrm{Z}_{2}=\frac{1}{2}[\mathrm{~A}, \mathrm{~B}] \\
& \left.\mathrm{Z}_{3}=\frac{1}{12}\{[\mathrm{~A},[\mathrm{~A}, \mathrm{~B}]]+[\mathrm{A}, \mathrm{B}], \mathrm{B}]\right\}
\end{aligned}
$$

A expressäo dual, conhecida como fórmula de Zassenhaus [34, 23], é dada por:

$$
e^{A \div B}=e^{A} e^{B} e^{C_{z}} \ldots e^{C_{n}} \ldots
$$

onde, $\mathrm{C}_{n}$ constitui uma soma de produtos de comutadores de n-ésimo grau de $\mathrm{A}$ e B. 
Suzuki, [27], estudou a convergência de 3.8 e deu uma forma sistemática para obtençăo dos operadores $\mathrm{C}_{n}$, cujos termos de menor ordem sāo:

$$
\begin{aligned}
& C_{2}=-\frac{1}{2}[A, B] \\
& C_{3}=\frac{1}{3}[B,[A, B]]+\frac{1}{6}[A,[A, B]]
\end{aligned}
$$

\subsubsection{Exemplo - O operador Deslocamento unitário de Glau- ber}

Seja: $\mathbf{A}=\alpha x \hat{a}_{1}^{\dagger}$ e $\mathrm{B}=-\alpha^{*} \hat{\mathrm{a}}_{1}$.

Com isto, usando a fórmula BCH 3.7 , onde

$$
\begin{aligned}
& \mathrm{Z}_{1}=\mathrm{B}=-\alpha^{*} \hat{\mathbf{a}}_{1} \\
& \mathrm{Z}_{2}=\frac{1}{2}[\mathrm{~A}, \mathrm{~B}\}=\frac{1}{2}\left[\alpha \hat{\mathrm{a}}_{1}^{\dagger},-\alpha^{*} \hat{\mathrm{A}}_{1}\right]=\frac{1}{2}|\alpha|^{2} \\
& \mathrm{Z}_{3}=0, \text { pois, } \quad\left[A_{1}\left[A_{1} \mathrm{~B}\right]\right]=\left[\boldsymbol{\alpha a}_{1},|\alpha|^{2}\right]=0 \\
& \mathbf{C}=\mathbf{A}+\mathbf{Z}_{1}+\mathbf{Z}_{2}+\ldots+\mathbf{Z}_{n}+\ldots=\alpha \tilde{a}_{1}^{1}-\alpha^{*} \hat{\mathbf{a}}_{1}+\frac{1}{2}|\alpha|^{2} \text {. }
\end{aligned}
$$

podemos obter o operador unitário de deslocamento de Glauber

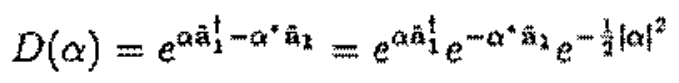

\subsection{Desacoplamento e ordenamento de operado- res}

Existem diversas técnicas para desacoplar uma exponencial de elementos de uma álgebra de Lie em produtos de exponenciais destes elementos.

A.s técnicas que iremos ressaltar são:

\subsubsection{Técnica matricial}

A técnica matricial, introduzida por Baker e Hausdorfl e redescobertas por Gilmore $[28,29]$, utiliza a representaçăo matricial de menor dimensionalidade dos elementos de uma álgebra de Lie, expressando as exponenciais destes elementos por neio de uma série de potências de matrizes.

\subsubsection{Exemplo}

Uma importante classe de sistemas de interesse em mecânica quântica é classe de operadores que representam a álgebra $S U(2)$ de monento angular. Az relaçoes que definem essa álgebra sảo:

$$
\left[J_{x:} J_{ \pm}\right]= \pm J_{ \pm} ; \quad\left[J_{i}, J_{-1}\right]=2 J_{x}
$$


Uma representação bi-dimensional dessa álgebra é dada pelas seguintes matrizes

$$
y_{+}=\left(\begin{array}{ll}
0 & 1 \\
0 & 0
\end{array}\right), J_{-}=\left(\begin{array}{ll}
0 & 0 \\
1 & 0
\end{array}\right), J_{z}=\left(\begin{array}{cc}
\frac{1}{2} & 0 \\
0 & -\frac{1}{2}
\end{array}\right) .
$$

Podemos escrever o exponencial de uma combinação linear de operadores em forma matricial

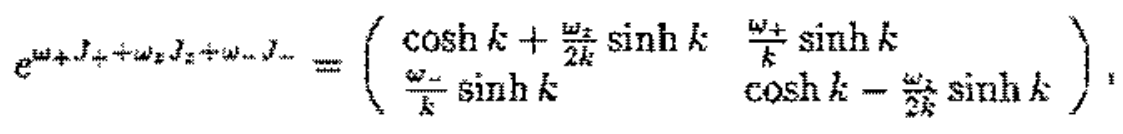

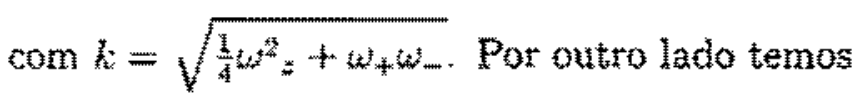

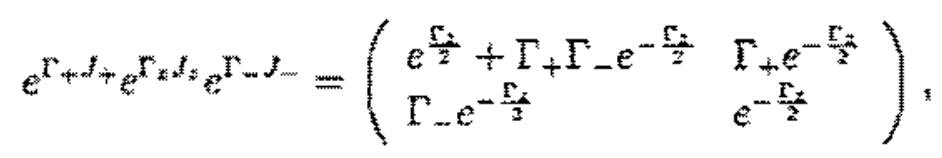

onde os fatores $\Gamma$ são dados em termos dos fatores $w$ e $k$ como segue

$$
\Gamma_{z}=\ln \left(\frac{2 k}{2 k \cosh k-\omega_{z} \sinh k}\right), \quad \Gamma_{+}=\frac{\omega_{+} \sinh k}{k} \Gamma_{z}, \Gamma_{-}=\frac{\omega_{-} \sinh k}{k} \Gamma_{z}
$$

\subsubsection{Técnica de Witschel e Técnica por derivaçāo de parâmetros}

A técnica de Witschel [23], basea-se nas transformações de similaridade de Hausdorff, 3.5 e 3.6, que é aplicada sucessivanente permitindo obter un conjunto de equaçōes algébricas cuja solução fornece os coeficientes de desacoplamento.

A definiçäo da derivada de um operador exponencial em relaçäos a um parâmetro foi proposta por Wilcox [33]. Fla objetiva determinar os coeficientes, $\beta_{i}$, que permitem expressar uma exponencial de combinaçōes lineares de operadores $\alpha_{1} A_{1}+$ $\alpha_{2} \mathrm{~A}_{2}+\ldots \alpha_{n} \mathrm{~A}_{n}$, em um produto de exponenciais de operadores, en qualquer ordem.

$$
e^{\alpha_{1} \hat{A}_{3}+\alpha_{3} \hat{A}_{2}+\ldots \alpha_{n} \hat{A}_{B}}=e^{B_{1} \hat{A}_{1}} e^{B_{2} \hat{A}_{2}} \ldots e^{B_{n} \hat{A}_{n}}
$$

Para tanto, as fórmulas de Baker-Campbell-Hausdorffe de Zassenkaus, säo suficientemente gerais, porkm a efetiva fatoraçāo das exponenciais dependem da álgebra dos operadores.

A aplicaçẫo đă técnica por derivação de parâmetros [14] consiste em:

- multiplicar a soma, $\alpha_{1} \mathbf{A}_{1}+\alpha_{2} \mathbf{A}_{2}+\ldots \alpha_{n} \mathbf{A}_{n}$ por um parâmetro, $t$ por exernplo, fazendo com que os coeficientes $\beta_{i}$ sejam funçōes destes parämetros, ou seja,

$$
e^{\sum_{i=1}^{n} a_{i} A_{i} t}=\prod_{i=1}^{n} e^{\beta_{i}(t) A_{1}}
$$


- derivar em relação ao parâmetro, $t$,

$$
\begin{gathered}
\sum_{i=1}^{n} \alpha_{i} \mathbf{A}_{i}\left(e^{\sum_{t=1}^{n} \alpha_{1} \mathbf{A}_{i} t}\right)=\quad \dot{\beta}_{1}(t) \mathbf{A}_{1} \prod_{i=1}^{n} e^{\beta_{i}(t) \mathbf{A}_{1}}+\dot{\beta}_{2}(t) e^{\beta_{1}(t) \mathbf{A}_{1}} \mathbf{A}_{2} \prod_{i=2}^{n} e^{\beta_{i}(t) \mathbf{A}_{i}}+ \\
\ldots \dot{\beta}_{n}(t) \prod_{i=1}^{n-1} e^{\beta_{1}(t) \mathbf{A}_{i}} \mathbf{A}_{n} e^{\beta_{1}(t) \mathbf{A}_{n}}
\end{gathered}
$$

- Usar a transformação de semelhança 3.5 (para o segundo termo por exemplo)

$$
\begin{aligned}
e^{\beta_{1}(t) \mathbf{A}_{4}} \mathbf{A}_{2} & =e^{\beta_{1}(t) \mathbf{A}_{1}} \mathbf{A}_{2} e^{-\beta_{1}(t) \mathbf{A}_{2}} e^{\beta_{1}(t) \mathbf{A}_{1}}=\sum_{n} \frac{\beta_{1}^{n}(t)}{n !}\left[\mathbf{A}_{1}, \cdot\right]^{n} \mathbf{A}_{2} e^{\beta_{1}(t) \mathbf{A}_{1}} \\
& \equiv \mathbf{F}_{2}\left(\beta_{1}(t),\left\{\mathbf{A}_{i}\right\}\right) e^{\beta_{1}(t) \mathbf{A}_{1}} \equiv \mathbf{F}_{2} e^{\beta_{1}(t) \mathbf{A}_{1}}
\end{aligned}
$$

analogamente para os termos seguintes,

$$
\mathbf{F}_{3} \equiv \mathbf{F}_{3}\left(\beta_{1}(t), \beta_{2}(t),\left\{\mathbf{A}_{i}\right\}\right) ; \quad \ldots \quad \mathbf{F}_{n} \equiv \mathbf{F}_{n}\left(\beta_{1}(t), \beta_{2}(t), . . \beta_{n-1}(t),\left\{\mathbf{A}_{\mathbf{i}}\right\}\right)
$$

- e escrever

$$
\begin{aligned}
\sum_{i=1}^{n} \alpha_{i} \mathbf{A}_{i}\left(e^{\sum_{i=1}^{n} \alpha_{1} \mathbf{A}_{i} t}\right) & =\left(\dot{\beta}_{1}(t) \mathbf{A}_{1}+\dot{\beta}_{2}(t) \mathbf{F}_{2}+\ldots+\dot{\beta}_{n}(t) \mathbf{F}_{n}\right) \odot \\
& \odot\left(e^{\sum_{i=1}^{n} \alpha_{i} \mathbf{A}_{i} t}\right)
\end{aligned}
$$

ou ainda

$$
\sum_{i=1}^{n} \alpha_{i} \mathbf{A}_{i}=\dot{\beta}_{1}(t) \mathbf{A}_{1}+\dot{\beta}_{2}(t) \mathrm{F}_{2}+\ldots+\dot{\beta}_{n}(t) \mathrm{F}_{n}
$$

onde, ao compararmos os coeficientes dos operadores $\left\{\mathbf{A}_{\boldsymbol{i}}\right\}$, iremos obter um sistema de equaçōes diferenciais, cuja soluçāo nos fornece os coeficientes $\beta_{i}$, com a condição inicial $\beta_{i}(t=0)=0$.

É importante notar que se desacoplarmos numa ordem diferente, iremos obter diferentes equaçōes diferenciais que poderão ser mais fáceis ou mais difíceis de serem resolvidas. 


\subsubsection{Exemplo: $O$ oscilador harmonnico forçado dissipativo}

Vamos tratar aqui um exemplo muito importante, o de um oscilador harmonico forçado e acoplado a um banho frio. Por simplicidade, vamos adotar também, como condiçẫo inicial un estado coerente.

A equaçăo que governa este sistema pode ser escrita da 1.27 onde $\mathrm{H}_{S}$ e dado por

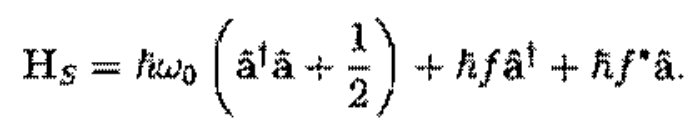

Com isto teremos

$$
\frac{d}{d t} \rho(t)=\mathcal{L} \rho(t) \longrightarrow \rho(t)=e^{L t} \rho(0)
$$

onde

$$
\mathcal{L}=-i \omega_{0}\left[\hat{\mathbf{a}}^{\dagger} \tilde{\mathbf{a}}_{\cdot} \cdot\right]-i\left[f \hat{\mathbf{a}}^{\dagger}+f^{*} \hat{\mathbf{a}}, \cdot\right]+k\left(2 \hat{\mathbf{a}} \cdot \hat{\mathbf{a}}^{\dagger}-\hat{\mathbf{a}}^{\dagger} \hat{\mathbf{a}}-\cdot^{\dagger} \hat{\mathbf{a}}^{\dagger} \hat{\mathbf{a}}\right)
$$

Através das relaçós de conutação B.1, verificamos que a álgebra, dada pelo liouvilliano da equação anterior, se fecha. Com isto, podemos ordenar essa exponencial como um produto de seus elementos,

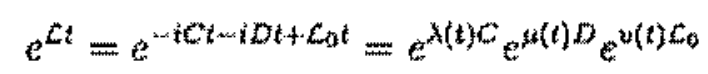

onde

$$
\begin{aligned}
& Q=f\left(\hat{a}^{\dagger} \cdot-\hat{a}^{\dagger}\right), \quad D=f^{*}(\hat{a} \cdot-\hat{a}),
\end{aligned}
$$

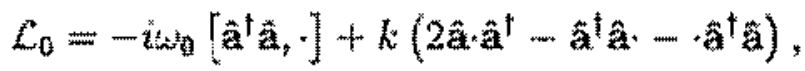

$$
\begin{aligned}
& \lambda(0)=\mu(0)=v(0)=0, \\
& {\left[\mathcal{C}_{a}, C\right]=-\left(\hat{u}_{0}+k\right) C} \\
& {\left[c_{\hat{\theta}}, D\right]=\left(i_{i}, k\right) D} \\
& {[C, D]=0}
\end{aligned}
$$

Derivando en relação ao parầmetro tobtemos:

$$
C e^{L t}=(\lambda(t) C+\mu(t) D) e^{L t}+\dot{v}(t) e^{\lambda(t) C} e^{u(t) D} C_{0} e^{v(t) C_{t}}
$$

Como

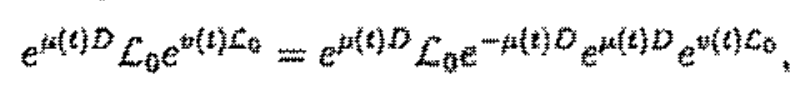

entāo

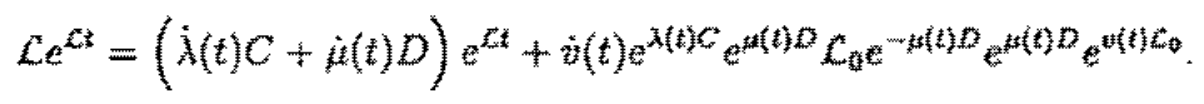


Aplicando o lema 3.5

$$
e^{m(t) D} C_{0} e^{-\mu(t) D}=\sum_{n} \frac{\mu^{n}}{n !}\left[D, E_{0}\right]=C_{0}-\mu\left(i \omega_{0}-k\right) D
$$

escreveremos

$$
\begin{aligned}
\mathcal{L} e^{C t} & =(\dot{\lambda}(t) C+\dot{\mu}(t) D) e^{\mathcal{L} t}+e^{\lambda(t) C}\left[\dot{v}(t) \mathcal{L}_{0}-\dot{v}(t) \mu\left(i \omega_{0}-k\right) D\right] e^{\mu(t) D} e^{v(t) \mathcal{L}_{0}} \\
& =\left[\dot{\lambda}(t) C+\dot{\mu}(t) D-\dot{v}(t)_{\mu}\left(i \omega_{0}-k\right) D\right] e^{\mathcal{L} t}+\dot{v}(t) e^{\lambda(t) C} \mathcal{L}_{0} e^{\mu(t) D} e^{v(t) \mathcal{L}_{0}}
\end{aligned}
$$

Como

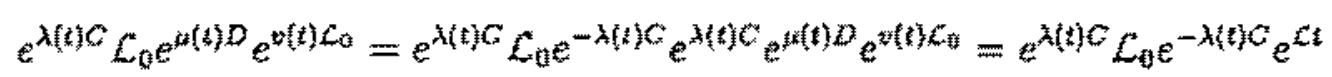

antaco

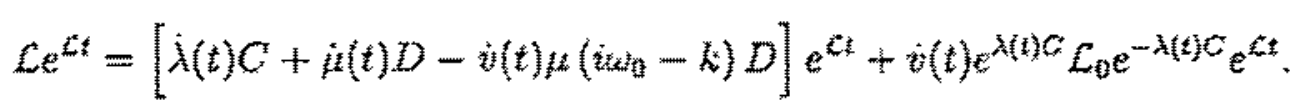

Aplicando o lema 3.5

$$
e^{\lambda(t) C} \mathcal{L}_{0} e^{-\lambda(t) C}=\sum_{n} \frac{\lambda^{n}}{n !}[C, \cdot]=C_{0}+\lambda\left(i t_{0}+k\right) C
$$

escreveremos

$$
\mathcal{L} e^{f t}=\left\{\left[\dot{\lambda}(t)+\dot{v}(t) \lambda\left(\dot{i} \omega_{0}+k\right)\right] C+\left(\dot{\mu}(t)-\dot{v}(t)_{\mu}\left(i \omega_{0}-k\right)\right] D+\dot{v}(t) \mathcal{L}_{0}\right\} e^{L t}
$$

Com isto

$$
C=\left[\dot{(t)}+\dot{v}(t) \lambda\left(i \omega_{0}+k\right)\right] C+\left[\mu(t)-v(t) \mu\left(u_{0}-k\right)\right] D+i(t) C_{0}
$$

Comparando os coeficientes obtemos

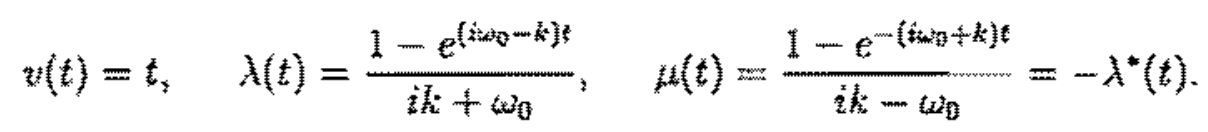

Ordenando fo na forma

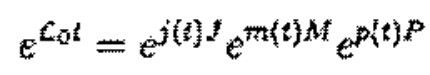

onde:

$$
\begin{aligned}
& J=\hat{\mathrm{a}} \cdot \hat{\mathrm{a}}^{\dagger}, \quad M=\hat{\mathrm{a}}^{\dagger} \hat{\mathrm{a}}{ }^{*}, \quad P=\mathrm{a}^{\dagger} \mathrm{a} \\
& {[J, M]=J, \quad[J, P]=J, \quad[P, M]=0 .}
\end{aligned}
$$

\footnotetext{
${ }^{1}$ ver relaçôes de comutação B.1
} 
Derivando en relaçẫo a parâmetro $t$ e procedendo de maneira análoga ao que foi feits anteriomente, obtemos

$$
j(t)=e^{2 k t}-1, \quad m(t)=-i \omega_{0} t-k t_{i} \quad p(t)=i \omega_{0} t-k t=m t^{2}(t)
$$

Assim, a evolução temporal de um oscilador harmonico forçado dissipativo

$$
\begin{aligned}
\rho(t) & =e^{\varepsilon t} \rho(0) \\
& =e^{\lambda(t) C} e^{\mu(t) D} e^{j(t) J} e^{r(t) M} e^{p(t) P} \rho(0),
\end{aligned}
$$

para um estado inicial coerente $\rho(0)=|\alpha\rangle\langle\alpha|$, é dada por"

$\rho(t)=\left|\alpha e^{\left(-i \omega_{0}-k\right)}+\frac{f}{i k+\omega_{0}}\left(1-e^{\left(i \omega_{0}-k\right) t}\right)\right\rangle\left\langle\alpha e^{\left(-i \omega_{0}-k\right)}+\frac{f}{i k+t_{0}}\left(1-e^{\left(i \omega_{0}-k\right) f}\right)\right|$

que é um estado coerente deslocado con amplitude que depende da razăo entre a fonte $(f)$ e a frequêtncia do oscilador $\left(\omega_{0}\right)$ com o coeficiente de dissipação $k$.

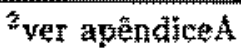




\section{Capítulo 4}

\section{Sistema aberto para dois osciladores}

Tendo considerado em capitulos anteriores possiveis tratamentos para a decoerência e dissipaçāo de dois sistemas quänticos acoplados, vamos mostrar aquí a possibilidade de tratar simultaneamente trẻs subsistemas. Escolhemos por simplicidade dois osciladores harmônicos acoplados entre sí, em contato com um reservatório frio e bombeados por fontes externas. Estendenos a técnica desenvolvida no capitulo 3 para o tratamento de dois osciladores.

\subsection{Dedução da equação mestra}

Nesta secção, deduziremos a equaçăo Mestra, considerando aproximaçōes adequadas para a dinâmica de dois osciladores acoplados entre sí, forçados externamente e em contato com um reservatório (banho) térmico. O Hamiltoniano total é dado por:

$$
\mathbf{H}=\mathbf{H}_{\mathrm{i}}+\mathrm{H}_{\mathrm{int}}
$$

onde:

$$
\mathbf{H}_{i n t}=\hbar \sum_{k}\left(\alpha_{k} \hat{c}_{k}^{\dagger} \hat{\mathbf{a}}+\alpha_{k}^{*} \hat{\mathrm{c}}_{k} \hat{\mathbf{a}}^{\dagger}\right)+\hbar \sum_{k}\left(\beta_{k} \hat{\mathrm{c}}_{k}^{\dagger} \hat{\mathbf{b}}+\beta_{k}^{*} \hat{\mathbf{c}}_{k} \hat{\mathbf{b}}^{\dagger}\right)
$$

é o Hamiltoniano de interação entre os osciladores representados pelos operadores ât, $\hat{\mathrm{a}}, \hat{\mathrm{b}}, \hat{\mathrm{b}}, \mathrm{o}$ reservatório representado pelos operadores $\hat{\mathrm{c}}_{k}^{\dagger}, \hat{\mathrm{c}}_{k}$ e $\alpha_{\mathrm{k}_{1}} p_{k}$ são constantes de acoplamento. $O$ hamiltoniano livre $\boldsymbol{H}_{0}$, ê

$$
\mathrm{H}_{0}=\mathrm{H}_{S^{z}}+\mathrm{H}_{R}, \quad \mathrm{H}_{S^{z}}=\mathbf{H}_{S}+\mathbf{H}_{i}+\mathrm{H}_{f}
$$

onde

$$
\mathbf{H}_{R}=\sum_{k} \hbar \omega_{k}\left(\hat{c}_{k} \hat{c}_{k}+\frac{1}{2}\right)
$$


é o Hamiltoniano do reservatório,

$$
\mathbf{H}_{s}=\mathbf{H}_{a}+\mathbf{H}_{b}=\hbar \omega_{\mathfrak{a}}\left(\hat{\mathbf{a}}^{\dagger} \hat{\mathbf{a}}+\frac{1}{2}\right)+\hbar w_{b}\left(\tilde{b}^{\dagger} \hat{\mathbf{b}}+\frac{1}{2}\right)
$$

é o Hamiltoniano dos osciladores,

$$
\mathrm{H}_{g}=h_{g}\left(\hat{\mathbf{a}}^{\dagger} \hat{\mathrm{b}}+\hat{\mathbf{a}} \hat{\mathrm{b}}^{\dagger}\right)
$$

é o Hamiltoniano de acoplamento entre os osciladoret,

$$
\mathrm{H}_{f}=\hbar f_{a} \hat{\mathrm{a}}^{\hat{i}}+h f_{a}^{*} \hat{a}+h f_{b} \hat{\mathrm{b}}^{\hat{b}}+\hbar f_{b}^{*} \hat{\mathrm{b}}
$$

é o Hamiltoniano de acoplamento dos osciladores com suas fontes externas. Note que os acoplamentos, tanto dos osciladores entre si quanto de cada um deles com o reservatório é do típo RWA. Além disso, os osciladores podem acoplar-se á reservatório com constantes de acoplamento $\alpha_{k}$ e $\beta_{k}$ diferentes e o bombeamento externo de energia, matematicamente expresso pelos termos de fonte, é independente para cada oscilador.

Tomando o traço sobre o teservatório da equação 1.20 , a equação da densidade reduzida para o sistenta constituido pelos dois osciladores "ab" será:

$$
\frac{d}{d t} \tilde{p}_{S}(t)=\frac{-i}{\hbar} t r_{R}\left[\tilde{H}_{i n t}(t), \tilde{\rho}(t)\right] \text {. }
$$

Note que esta não é uma equação diferencial fechada para $\tilde{\rho}_{S}(t)$, pois nela aparece $\tilde{p}(t)$. A idéia é obter uma equação fechada enn funçäo de $\widetilde{p}_{S}(t)$

$$
\frac{d}{d t} \tilde{p}_{S}(t)=\Lambda \tilde{p}_{s}(t)
$$

para isso, iremos determinar $A$ em função de $H_{\xi a}$ atravếs da evolução do operador densidade na forma integral, equação 1.21, truncando na ordem dominante. Partiremos, conforme jả mencionamos, da equaçăo 1.21 , interando-a uma vez:

$$
\frac{d}{d t} \widetilde{\rho}_{S}(t)=\frac{-i}{\hbar} t r_{R}\left[\widetilde{\mathbf{H}}_{i n t}(t), \tilde{\rho}(0)\right]+\frac{-1}{\hbar^{2}} t r_{R}\left[\tilde{\mathbf{H}}_{i n t}(t), \int_{0}^{t} d t^{\prime}\left[\widetilde{\mathbf{H}}_{i n t}\left(t^{\prime}\right), \vec{\rho}\left(t^{r}\right)\right]\right]
$$

onde,

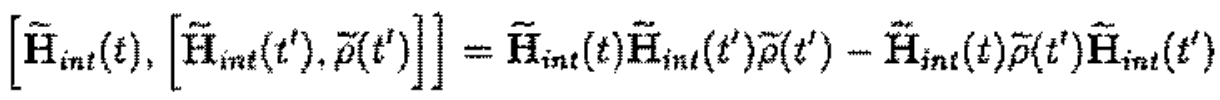

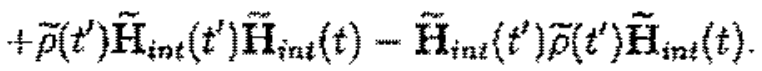

$\mathrm{Como}_{3}$ da equaçäo 1.0

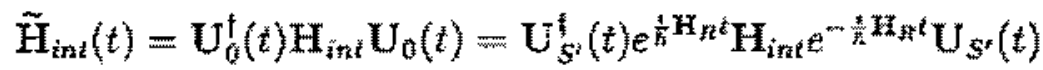


onde $\mathrm{U}_{S^{\prime}}(t)$ é o propagador relativo a hamiltoniana dependente do tempo $\mathrm{H}_{S^{\prime}}$, então

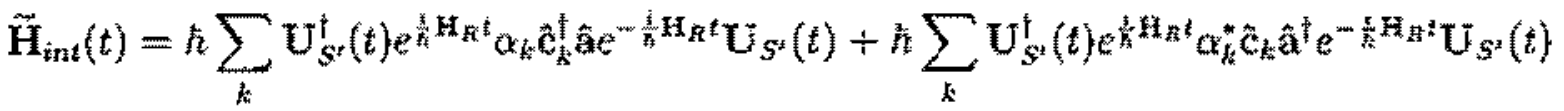

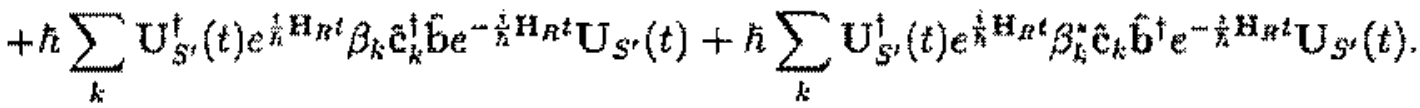

Com o objetivo de simplificar a notaçăo, definiremos

$$
\begin{aligned}
& \Gamma_{1}^{\dagger}(t) \equiv \hbar \sum_{k} e^{t \omega_{k} t} \alpha \alpha_{k} c_{k}^{\dagger}, \quad \Gamma_{2}(t) \equiv \hbar \sum_{k} e^{-i \omega_{k} t} \beta_{k}^{*} \hat{c}_{k},
\end{aligned}
$$

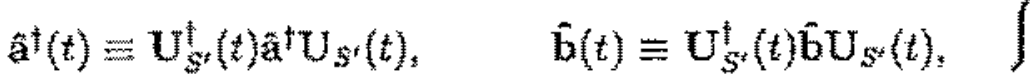

que podemos substituir em $\mathrm{H}_{\text {int }}$ obtendo:

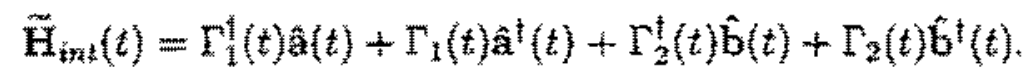

Substituindo no primeiro termo do duplo conutador da equação 4.2 , teremos:

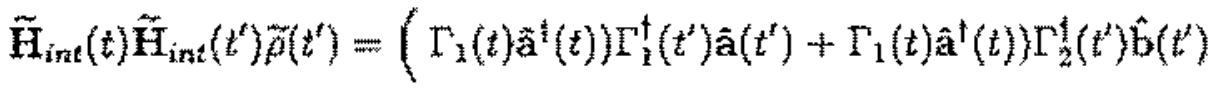

$$
\begin{aligned}
& +\Gamma_{2}(t) \hat{\mathrm{b}}^{\dagger}(t) \Gamma_{2}^{\dagger}\left(t^{\prime}\right) \hat{\mathrm{b}}\left(t^{\prime}\right)+\Gamma_{2}(t) \hat{\mathrm{b}}^{\dagger}(t) \Gamma_{1}^{\dagger}\left(t^{\prime}\right) \hat{\mathrm{a}}\left(t^{\prime}\right) \\
& +\Gamma_{1}^{\dagger}(t) \hat{a}(t) \Gamma_{1}\left(t^{\prime}\right) \hat{a}^{\dagger}\left(t^{\prime}\right)+\Gamma_{1}^{\dagger}(t) \hat{a}(t) \Gamma_{2}\left(t^{\prime}\right) \tilde{b}^{\dagger}\left(t^{\prime}\right) \\
& \left.+\Gamma_{2}^{\dagger}(t) \hat{\mathrm{b}}(t) \Gamma_{2}\left(t^{\prime}\right) \hat{\mathrm{b}}^{\dagger}\left(t^{\prime}\right)+\Gamma_{2}^{\dagger}(t) \hat{\mathrm{b}}(t) \Gamma_{1}\left(t^{\prime}\right) \hat{\mathrm{a}}^{\dagger}\left(t^{\prime}\right)\right) \not{\rho}\left(t^{\prime}\right) \\
& +\left(\Gamma_{1}^{\prime}(t) \hat{a}(t) \Gamma_{1}^{\dagger}\left(t^{\prime}\right) \hat{a}\left(t^{\prime}\right)+\Gamma_{1}^{\dagger}(t) \hat{a}(t) \Gamma_{2}^{\dagger}\left(t^{\prime}\right) \hat{b}\left(t^{\prime}\right)\right. \\
& +\Gamma_{2}^{\dagger}(t) \hat{\mathrm{b}}(t) \Gamma_{2}^{\dagger}\left(t^{\prime}\right) \hat{\mathrm{b}}\left(t^{\prime}\right)+\Gamma_{2}^{\dagger}(t) \hat{\mathrm{b}}(t) \Gamma_{1}^{\dagger}\left(t^{\prime}\right) \hat{\mathrm{a}}\left(t^{\prime}\right) \\
& \left.\left.+\Gamma_{1}(t) \hat{a}^{\dagger}(t)\right) \Gamma_{1}\left(t^{t}\right) \hat{a}^{\dagger}\left(t^{t}\right)+\Gamma_{1}(t) \vec{a}^{t}(t)\right) \Gamma_{2}\left(t^{t}\right) \hat{b}^{\dagger}\left(t^{t}\right) \\
& \left.+\Gamma_{2}(t) \hat{b}^{\dagger}(t) \Gamma_{2}\left(t^{t}\right) \hat{b}^{\dagger}\left(t^{t}\right)+\Gamma_{2}(t) \hat{b}^{\dagger}(t) \Gamma_{1}\left(t^{t}\right) \mathrm{a}^{\dagger}\left(t^{z}\right)\right) \tilde{p}\left(t^{t}\right)
\end{aligned}
$$

Para compactar a notaçäo, definiremos $\hat{\mathrm{a}}(t) \equiv \hat{\mathrm{o}}_{1}(t), \overrightarrow{\mathrm{b}}(t) \equiv \hat{\mathrm{o}}_{2}(t)$ e escreveremos:

$$
\begin{aligned}
\tilde{\mathrm{H}}_{\mathrm{int}(}(t) \tilde{\mathrm{H}}_{i n t}\left(t^{\prime}\right) \tilde{\rho}\left(t^{\prime}\right)= & \sum_{i, j=1}^{2}\left(\Gamma_{i}(t) \Gamma_{j}^{\dagger}\left(t^{\prime}\right) \hat{o}_{i}^{\dagger}(t) \hat{o}_{j}\left(t^{\prime}\right)+\Gamma_{i}^{\dagger}(t) \Gamma_{j}\left(t^{\prime}\right) \hat{o}_{i}(t) \hat{o}_{j}^{\dagger}\left(t^{t}\right)\right) \tilde{\rho}\left(t^{t}\right) \\
& +\sum_{i, j=1}^{2}\left(\Gamma_{i}^{\dagger}(t) \Gamma_{j}^{\dagger}\left(t^{\prime}\right) \hat{o}_{i}(t) \hat{o}_{j}\left(t^{\prime}\right)+\Gamma_{i}(t) \Gamma_{j}\left(t^{\prime}\right) \hat{o}_{i}^{\dagger}(t) \hat{o}_{j}^{\dagger}\left(t^{\prime}\right)\right) \tilde{\rho}\left(t^{\prime}\right)
\end{aligned}
$$

Podemos agora, proceder de maneira análoga para os outros termos e reescrevera 
equaçào 4.1, na forma:

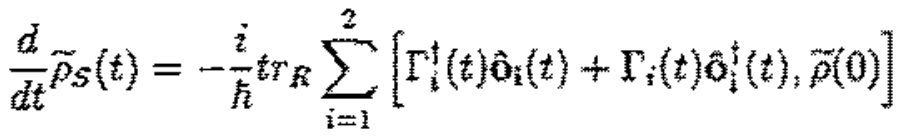

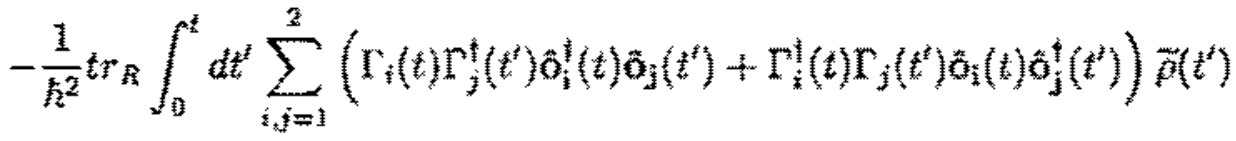

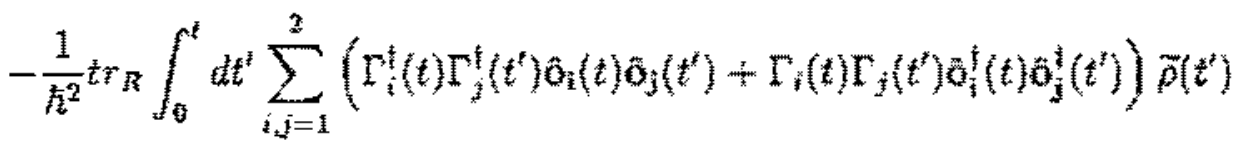

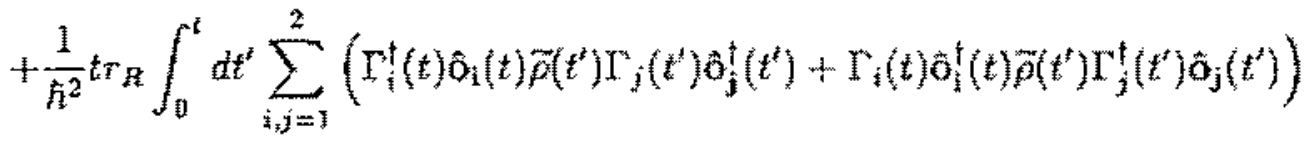

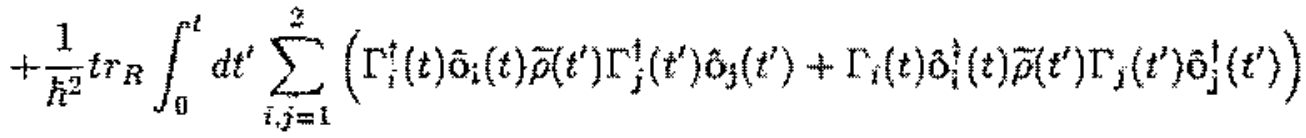

$$
\begin{aligned}
& -\frac{1}{\hbar^{2}} t r_{n} \int_{0}^{t} d t^{\prime} \sum_{i, j=1}^{2} \tilde{\rho}\left(t^{\prime}\right)\left(\Gamma_{i}^{\dagger}\left(t^{\prime}\right) \Gamma_{j}(t) \hat{o}_{i}\left(t^{\prime}\right) \hat{o}_{j}^{\dagger}(t)+\Gamma_{i}\left(t^{\prime}\right) \Gamma_{j}^{\dagger}(t) \hat{o}_{i}^{\ddagger}\left(t^{\prime}\right) \hat{o}_{j}(t)\right) \\
& -\frac{1}{\hbar^{2}} t_{R} \int_{0}^{t} d t^{\prime} \sum_{i, j=1}^{2} \tilde{\rho}\left(t^{\prime}\right)\left(\Gamma_{i}^{\dagger}\left(t^{\prime}\right) \Gamma_{j}^{\dagger}(t) \hat{o}_{i}\left(t^{\prime}\right) \hat{\sigma}_{\mathrm{j}}(t)+\Gamma_{i}\left(t^{\prime}\right) \Gamma_{j}(t) \hat{\sigma}_{j}^{\dagger}\left(t^{\prime}\right) \hat{o}_{j}^{\dagger}(t)\right) \\
& +\frac{1}{\hbar^{2}} t r_{R} \int_{0}^{t} d t^{t} \sum_{i, j=1}^{2}\left(\Gamma_{i}^{\dagger}\left(t^{\prime}\right) \hat{\sigma}_{i}\left(t^{\prime}\right) \tilde{p}^{\prime}\left(t^{\prime}\right) \Gamma_{j}(t) \hat{o}_{j}^{\dagger}(t)+\Gamma_{i}\left(t^{\prime}\right) \hat{o}_{i}^{t}\left(t^{\prime}\right) p\left(t^{\prime}\right) \Gamma_{j}^{\dagger}(t) \hat{o}_{j}(t)\right)
\end{aligned}
$$

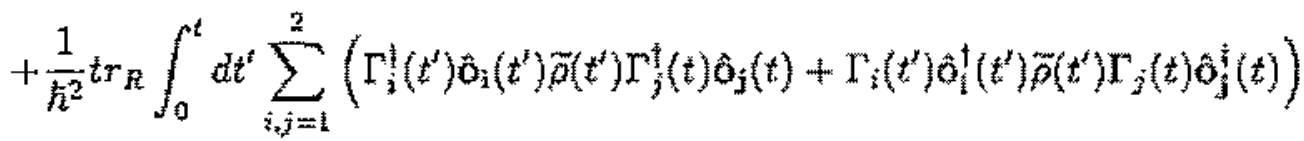

Esta equação ainda exata, não fechada esem soluçẩo. Para torná-la analiticamente tratável faremos as seguintes hipóteses dinâmicas:

1. Hipótese para $\tilde{\rho}^{t}$ ) descorrelacionado e un banho estätico.

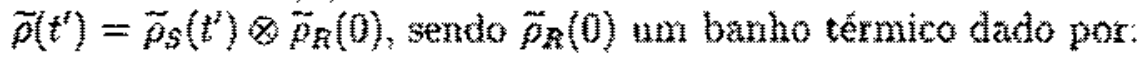

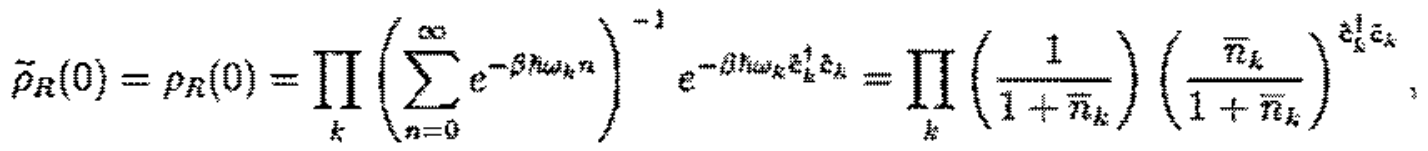

onde $\beta=\left(k_{B} T\right)^{-1}, k_{B}$, a constante de Boltamann, $T$, temperatura, e $\bar{n}_{k} \varepsilon$ 
o número médio de fótons no reservatório do k-ésimo modo, dado pela distribuição de Planck, ou seja,

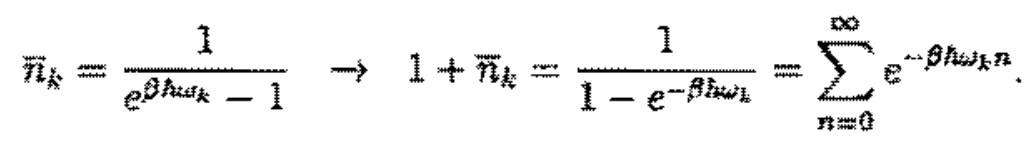

Consequentemente, wsando uma base de estado de Fock, podemos concluir quanto aos tracos sobre as variáveis do reservatório da equação 4.4

$$
\begin{aligned}
& \operatorname{Tr} p_{R}(0) \hat{c}_{k}^{\dagger m} \hat{c}_{k^{t}}^{n}=\left\langle\hat{c}_{k}^{\dagger m} \hat{c}_{k}^{n}\right\rangle=\left\langle\left(\hat{c}_{k}^{\dagger m} \hat{c}_{k}^{m}\right\rangle \delta_{k k^{\prime}} \quad \delta_{m n}\right. \\
& \downarrow \\
& \left\langle\Gamma_{k}(t) \Gamma_{k}\left(t^{f}\right)\right)=\left\langle\Gamma_{k}^{t}\left(t^{2}\right) \Gamma_{k}^{t}(t)\right\rangle=\left\langle\Gamma_{k}(t)\right\rangle=\left|\Gamma_{k}^{t}\left(t^{\prime}\right)\right\rangle=0,
\end{aligned}
$$

Fisicamente esta hipótese assume que o banho näo é afetado pela presença do subsistema $S$, supostamente muito menor que o reservatório, implicando implicitamente que a interaçăo entre ambos é fraca. A própria validade dessa aproximactio pode ser um critario quanto a força do acoplamento entre $S$ e o banho. Essa aproximaçẫo e näo perturvativa no sentido en que todas as

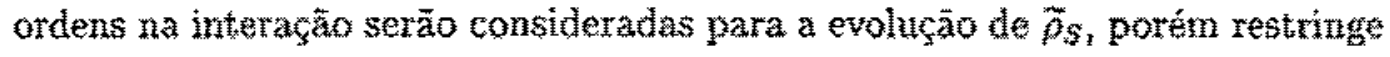
a classe de denaidades reduzidas aquelas que satisfazem ao ansatz proposto como hupótese.

2. Hipótese de ruído branco onde o reservatório é considerado infinito, fazendo com que o intervalo entre dois modos adjacentes atinja o limite contínuo. Assim, podewse definir una densidade de modos, $D(\omega)$, do reservatónio onde $D(w)$ du fomece o rúmero de modos no intervalo comprendido entre $w$ $w+d$ da lazer as seguintes substitutiches:

$$
\begin{aligned}
& \sum_{k} \rightarrow \int_{0}^{\infty} D(\omega) d \omega \\
& \bar{n}_{k} \rightarrow \bar{n}(\omega) \\
& \alpha_{k} \rightarrow \alpha(\omega) \\
& \beta_{k} \rightarrow p(\omega)
\end{aligned}
$$

Na hipotese de rufdo branco, é suposto que $D(\omega)|\alpha(\omega)|^{2}, D(\omega) \alpha(\omega) \beta^{*}(\omega)$, $D(\omega)|\alpha(\omega)|^{2} \bar{n}(\omega), \ldots$ sảo funçóes suficientemente suaves de $\omega$. Com isto, a transformada de Fourier dessas funçôe terá a forma de uma funçăo que decai de maneira täo rápida quanto mais suave forem estas funços, de tal modo que no limite em que as funçōes são complatamente planas, o tempo de decaimento de sua transformada tende a zero. 
Assim podemos modelar o banko na forma:

$$
\begin{aligned}
& D(\omega)|\alpha(\omega)|^{2} \approx \frac{k_{s}}{\pi^{2}}, \quad D(\omega)|\beta(\omega)|^{3} \approx \frac{k_{b}}{\pi},
\end{aligned}
$$

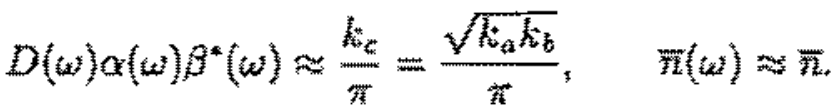

Esta hipotese é uzual e, como veremos, será responsâvel pelo carảter markoviano da equaçäo resultante.

A primeira hipótese, reduz a equaçāo, 4.4 a:

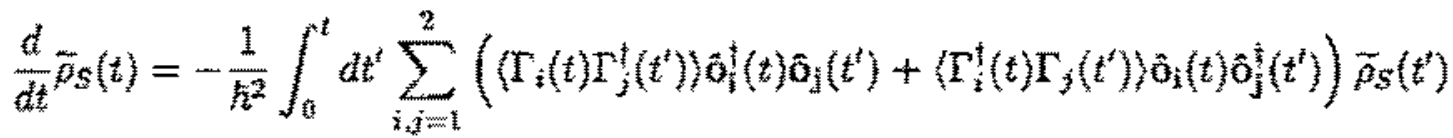

$$
\begin{aligned}
& +\frac{1}{h^{2}} \int_{0}^{t} d t^{\prime} \sum_{i, j=1}^{2}\left(\left\langle\Gamma_{j}\left(t^{\prime}\right) \Gamma_{i}^{\dagger}(t)\right\rangle \hat{\sigma}_{j}(t) \tilde{\rho}_{S}\left(t^{\prime}\right) \hat{\sigma}_{j}^{t}\left(t^{2}\right)+\left\langle\Gamma_{j}^{\dagger}\left(t^{\prime}\right) \Gamma_{i}(t)\right\rangle \hat{o}_{i}^{l}(t) \tilde{\rho}_{S}\left(t^{\prime}\right) \hat{o}_{j}\left(t^{\prime}\right)\right)
\end{aligned}
$$

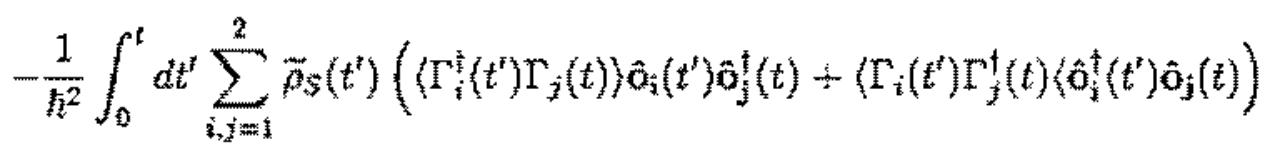

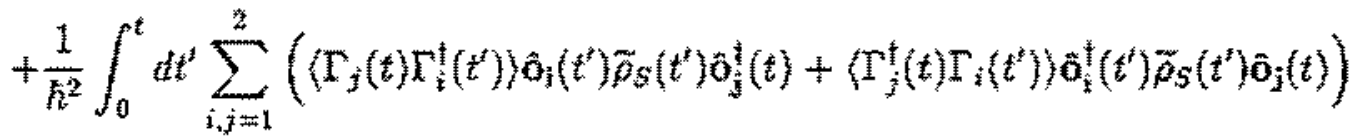

Resolvendo o primeiro termo da equacäo anterior obtemos

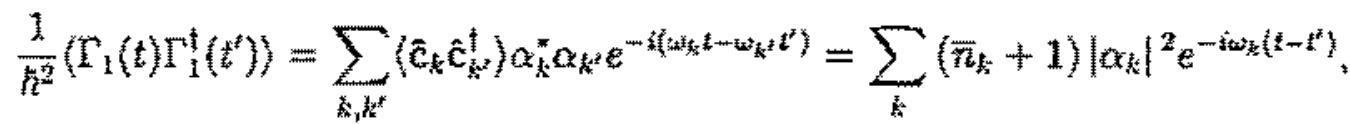

$$
\begin{aligned}
& \frac{1}{\hbar^{2}}\left\langle\Gamma_{\mathbf{i}}(t) \Gamma_{2}^{\dagger}\left(t^{\prime}\right)\right\rangle=\sum_{k, k^{\prime}}\left\langle\hat{\mathbf{c}}_{k} \hat{c}_{k^{\prime}}^{\dagger}\right\rangle \alpha_{k}^{*} \beta_{k^{\prime}} e^{-i\left(\omega_{k_{k}} t-\omega_{k^{\prime}} t^{2}\right)}=\sum_{k}\left(\bar{n}_{k}+1\right) \alpha_{k}^{*} \beta_{k^{\prime}} e^{-i \omega_{k}\left(t-t^{*}\right)}
\end{aligned}
$$

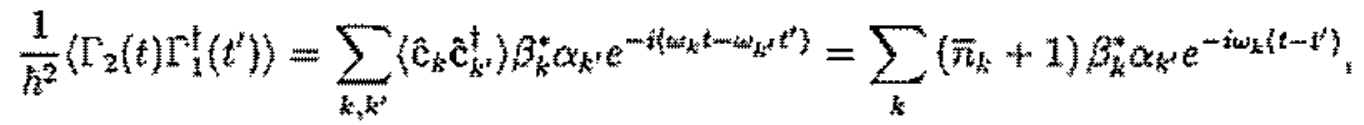

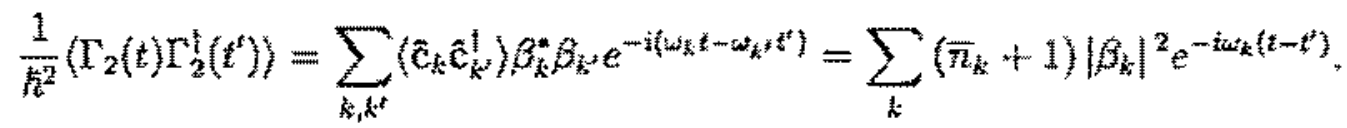

Da segunda hipótese, equaçäo 4.6 , teremos:

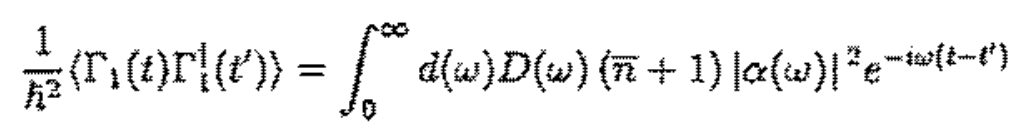




$$
\begin{aligned}
& \downarrow \\
& \frac{1}{h^{2}}\left\langle\Gamma_{1}(t) \Gamma_{1}^{\dagger}\left(t^{\prime}\right)\right\rangle=(\bar{n}+1) \frac{k_{a}}{\pi} \int_{0}^{\infty} d(\omega) e^{-i \omega\left(t-t^{\prime}\right)} \\
& \frac{1}{n^{2}}\left(\Gamma_{1}(t) \Gamma_{2}^{t}\left(t^{t}\right)\right)=\int_{0}^{\infty} d(\omega) D(\omega)(n+1) \alpha^{*} \beta e^{-i \omega\left(t-t^{\prime}\right)} \\
& \downarrow
\end{aligned}
$$

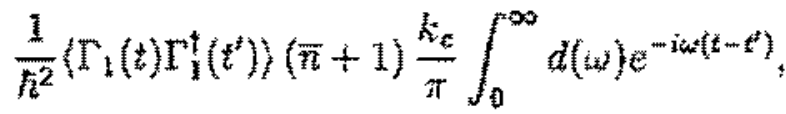

$$
\begin{aligned}
& \frac{1}{h^{2}}\left(\Gamma_{2}(t) \Gamma_{1}^{\dagger}\left(t^{\prime}\right)\right)=\int_{0}^{\infty} d(\omega) D(\omega)(\bar{n}+1) \alpha \beta^{*} e^{-i \omega\left(t-t^{\prime}\right)} \\
& \downarrow \\
& \frac{1}{h^{2}}\left(\Gamma_{2}(t) \Gamma_{1}^{\dagger}\left(t^{\prime}\right)\right)=(\bar{n}+1) \frac{k_{c}}{\pi} \int_{0}^{\infty} d(\omega) e^{-i \omega\left(l-l^{\prime}\right)}
\end{aligned}
$$

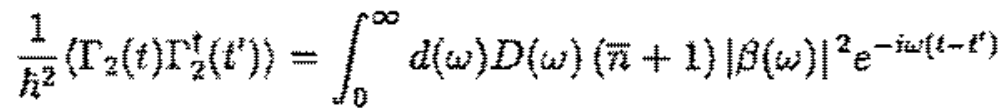

$$
\begin{aligned}
& \downarrow \\
& \frac{1}{\hbar^{2}}\left(\Gamma_{2}(t) \Gamma_{2}^{t}\left(t^{\prime}\right)\right)=(\bar{n}+1) \frac{k_{2}}{\pi} \int_{0}^{\infty} d(\omega) t^{\omega i \omega\left(t-t^{t}\right)}
\end{aligned}
$$

Uma vez que

$$
\left.\int_{0}^{\infty} d t\right) e^{ \pm i \omega t}=\pi \delta(t) \pm i P\left(\frac{1}{i}\right)
$$

onde $P\left(\frac{1}{1}\right)$ denota a parte principal de $\left(\frac{1}{2}\right)$, podemos excrever:

$$
\begin{aligned}
& \left\langle\Gamma_{1}(t) \Gamma_{1}^{t}\left(t^{t}\right)\right\rangle=\left(\Gamma_{1}\left(t^{t}\right) \Gamma_{1}^{\dagger}(t)\right\rangle=h^{2}(\bar{n}+1) k_{d} \delta\left(t-t^{t}\right) \\
& \left(\Gamma_{1}(t) \Gamma_{2}^{t}\left(t^{t}\right)\right\rangle=\left(\Gamma_{1}\left(t^{t}\right) \Gamma_{2}^{\dagger}(t)\right\rangle=h^{2}(\bar{n}+1) k_{c} \delta\left(t-t^{\prime}\right) \\
& \left\langle\Gamma_{2}(t) \Gamma_{1}^{\dagger}\left(t^{\prime}\right)\right\rangle=\left\langle\Gamma_{2}\left(t^{\prime}\right) \Gamma_{1}^{\dagger}(t)\right\rangle=h^{2}(\bar{n}+1) k_{c} \delta\left(t-t^{\prime}\right) \\
& \left\langle\Gamma_{2}(t) \Gamma_{2}^{\dagger}\left(t^{\prime}\right)\right\rangle=\left\langle\Gamma_{2}\left(t^{\prime}\right) \Gamma_{2}^{\dagger}(t)\right\rangle=h^{2}(\bar{n}+1) k_{b} \delta\left(t-t^{\prime}\right) .
\end{aligned}
$$

Estas expressöes foram estritas sem as contribuiços da parte principal $P\left(\frac{1}{i}\right)$, cujo efeito sobre a dinamica a po năo é tratado aqui. Na literatura, ele e geralmente assumido como pequeno.

Resolvendo de manatra anăloga para os outros termos da equação 4.7 e retor nando à notaçăo original, teremos:

$$
\begin{aligned}
& \frac{d}{d t} \tilde{\rho}_{S}(t)=-\tilde{n}\left(k_{a} \tilde{a}(t) \hat{\mathbf{a}}^{\dagger}(t)+k_{b} \hat{\mathrm{b}}(t) \hat{\mathrm{b}}^{\dagger}(t)\right) \tilde{\rho}_{S}(t) \\
& -\bar{\pi} k_{c}\left(\hat{a}(t) \hat{\mathbf{b}}^{\dagger}(t)+\hat{\mathbf{b}}(t) \mathbf{a}^{\dagger}(t)\right) \tilde{\partial}_{s}(t)
\end{aligned}
$$




$$
\begin{aligned}
& -(\bar{n}+1)\left(k_{a} \hat{a}^{t}(t) \mathrm{a}(t) k_{b} \hat{b}^{t}(t) \hat{b}(t)\right) \hat{p}_{S}(t) \\
& -(n+1) k_{z}\left(a^{t}(t) b(t)+b^{t}(t) a(t)\right) \tilde{\rho}_{5}(t) \\
& +\bar{n}\left(k_{a} \hat{a}^{t}(t) p_{s}(t) \hat{a}(t)+k_{b} b^{t}(t) \overline{p s}(t) \hat{b}(t)\right)
\end{aligned}
$$

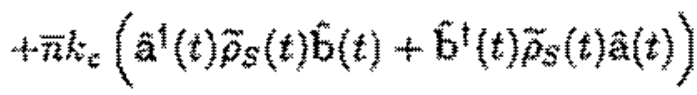

$$
\begin{aligned}
& +(\bar{n}+1)\left(k_{\mathrm{a}} \hat{\mathrm{a}}(t) \hat{\rho}_{S}(t) \hat{\mathrm{a}}^{\dagger}(t)+k_{b} \hat{\mathrm{b}}(t) \hat{\rho}_{S}(t) \hat{\mathrm{b}}^{\dagger}(t)\right) \\
& \div(\bar{n}+1) k_{e}\left(\hat{a}(t) \tilde{p}_{s}(t) \hat{b}^{\dagger}(t)+\vec{b}(t) \tilde{p}_{s}(t) \hat{a}^{\dagger}(t)\right) \\
& -(n) \tilde{\rho}_{S}(t)\left(k_{\mathrm{a}} \hat{\mathrm{a}}(t) \hat{\mathrm{a}}^{t}(t)+k_{b} \overrightarrow{\mathrm{b}}(t) \mathrm{b}^{\dagger}(t)\right) \\
& -(\bar{n}) \tilde{\rho}_{s}(t) k_{c}\left(\hat{a}(t) \hat{b}^{\dagger}(t)+\hat{b}(t) a^{\dagger}(t)\right) \\
& -(\tilde{n}+1) \tilde{\rho}_{S}(t)\left(k_{a} \hat{\mathrm{a}}^{t}(t) \hat{\mathrm{a}}(t)+k_{s} \hat{\mathrm{b}}^{\dagger}(t) \hat{\mathrm{b}}(t)\right) \\
& -(\bar{n}+1) \tilde{\rho}_{S}(t) k_{c}\left(\vec{a}^{\dagger}(t) \hat{\mathbf{b}}(t)+\hat{\mathbf{b}}^{\dagger}(t) \hat{\mathbf{a}}(t)\right) \\
& +\bar{n}\left(k_{a} \tilde{a}^{\dagger}(t) \tilde{\rho}_{S}(t) \hat{\mathbf{a}}(t)+k_{b} \overrightarrow{\mathrm{b}}^{\dagger}(t) \tilde{\rho}_{S}(t) \hat{\mathbf{b}}(t)\right) \\
& +\bar{n} k_{c}\left(\hat{a}^{\dagger}(t) \tilde{\rho}_{S}(t) \hat{\mathrm{b}}(t)+\hat{b}^{\dagger}(t) \tilde{\rho}_{S}(t) \tilde{a}(t)\right) \\
& +(\bar{n}+1)\left(k_{a} a(t) \tilde{p}_{s}(t) \hat{a}^{\dagger}(t)+k_{b} \vec{b}(t) \tilde{p}_{s}(t) \hat{b} t(t)\right) \\
& +(\bar{n}+1) k_{c}\left(\hat{\mathrm{a}}(t) \tilde{p}_{s}(t) \hat{\mathrm{b}}^{\dagger}(t)+\tilde{\mathrm{b}}(t) \bar{\rho}_{S}(t) \hat{\mathrm{a}}^{\dagger}(t)\right)
\end{aligned}
$$

Por outro lado:

1. Explicitando a representaçầo de interaçäo para $\widetilde{\rho}(t)$,

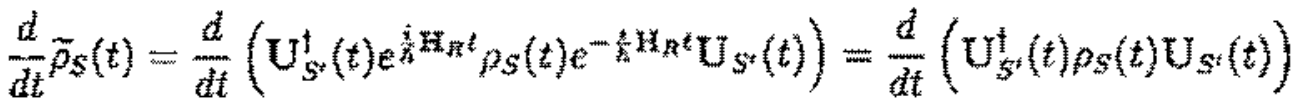

$$
\begin{aligned}
& =\frac{i}{\hbar}\left[\mathbf{H}_{s^{n}}, p_{s}(t)\right]+\mathbf{U}_{s^{\prime}}^{t}(t)\left(\frac{d}{d t} p s(t)\right) \mathbf{U}_{s^{\prime}}(t)
\end{aligned}
$$

chegaremos a

$$
\mathrm{U}_{s^{\prime}(t)}\left(\frac{d}{d t} \tilde{p}_{s}(t)\right) \mathrm{U}_{s^{\prime}}^{t}(t)=\frac{\dot{i}}{h}\left[\mathrm{H}_{S^{\prime}}, p_{s}(t)\right]+\frac{d}{d t} p_{s}(t) .
$$


2. Se tomarmos o primeiro termo da equaşäo 4.9 como exemplo e substituimos a relaçăo 4.3 ,

$$
\begin{aligned}
& \hat{\mathrm{a}}(\hat{t}) \hat{a}^{\dagger}(\underline{t}) p_{S}(t)=
\end{aligned}
$$

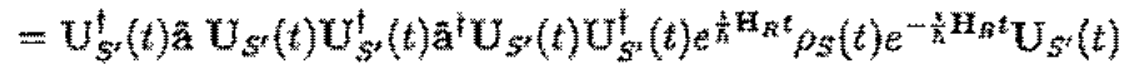

$$
\begin{aligned}
& =U_{S^{\prime}}^{\dagger}(t) a^{\dagger} p_{s}(t) U_{S^{\prime}}(t)
\end{aligned}
$$

conchiremos que

$$
\mathrm{U}_{S^{\prime}}(t) \hat{a}(t) \hat{a}^{\dagger}(t) \rho_{S}(t) \mathbf{U}_{S^{\prime}}^{\dagger}(t)=\operatorname{ă}^{\dagger} \rho_{S}(t)
$$

Procedendo de maneira análoga para os outros termos da equação 4.9 , concluiremos a idéia inicial desta seção e podemos escrever a equaçăo mestra markoviana para o sistema aberto, $S$,

$$
\begin{aligned}
& \frac{d}{d t} \rho_{S}(t)=-\frac{i}{h}\left[\mathbf{H}_{S^{x}}, p_{S}(t)\right] \\
& +K_{\mathrm{a}}(\bar{n}+1)\left(2 \hat{\mathbf{a}} \rho_{S}(t) \overrightarrow{\mathbf{a}}^{\dagger}-\hat{\mathbf{a}}^{\dagger} \hat{\mathrm{a}} \rho_{S}(t)-\rho_{S}(t) \hat{\mathbf{a}}^{\dagger} \hat{\mathbf{a}}\right) \\
& +k_{b}(\dddot{n}+1)\left(2 \hat{\mathbf{b}} \rho_{S}(t) \hat{\mathbf{b}}-\hat{\mathbf{b}}^{\dagger} \hat{\mathbf{b}}_{\rho_{S}}(t)-\rho_{s}(t) \hat{\mathbf{b}}^{\dagger} \hat{\mathbf{b}}\right) \\
& +k_{c}(\bar{n}+1)\left(2 \hat{a} \rho_{S}(t) \hat{\mathrm{b}}^{\dagger}-\mathbf{a}+\hat{b} \rho_{S}(t)-\rho_{S}(\hat{t}) \mathbf{a}^{\dagger} \hat{\mathrm{b}}\right) \\
& +k_{c}(\bar{n}+1)\left(2 \hat{\mathbf{b}} \rho_{S}(t) \hat{\mathbf{a}}^{\dagger}-\hat{\mathbf{b}}^{\dagger} \hat{\mathbf{a}} \rho_{S}(t)-\rho_{s}(t) \hat{\mathbf{b}}^{\dagger} \hat{\mathbf{a}}\right) \\
& +k_{s i} \bar{n}\left(2 \hat{a}^{\dagger} p_{s}(t) \hat{a}-\hat{a} \hat{a}^{\dagger} p_{s}(t)-p_{s}(t) \hat{a} \hat{a}^{\dagger}\right) \\
& +k_{b} \tilde{r}\left(\hat{b}^{\dagger} \rho_{s}(t) \hat{\mathbf{b}}-\hat{\mathbf{b}} \hat{\mathrm{b}}^{\dagger} \rho_{s}(t)-\rho_{s}(t) \hat{\mathbf{b}} \hat{\mathrm{b}}^{\dagger}\right) \\
& +h_{k} n\left(2 a^{\dagger} a_{S}(t) b-a b^{\dagger} p_{S}(t)-\rho_{s}(t) \hat{a} b^{t}\right) \\
& +k_{c} \bar{n}\left(2 \hat{b}^{\dagger} \rho_{S}(t) \hat{a}-\hat{\mathbf{b}} \hat{a}^{t} \rho_{S}(t)-\rho_{s}(t) \hat{\mathbf{b}} \hat{a}^{\dagger}\right) .
\end{aligned}
$$


O resultado para um banho com tempereturaeratura nula, $\bar{n} \rightarrow 0$, será:

$$
\begin{aligned}
& \frac{d}{d t} \rho_{S}(t)=-\frac{i}{\hbar}\left[\mathbf{H}_{S^{\prime}}, \rho_{S}(t)\right] \\
& +k_{a}\left(2 \hat{a} \rho_{S}(t) \hat{\mathbf{a}}^{\hat{i}}-\hat{a}^{\dagger} \hat{a} \rho_{S}(t)-\rho_{S}(t) \hat{a}^{\dagger} \hat{a}\right) \\
& +k_{z}\left(2 \hat{\mathbf{b}} \rho_{S}(t) \hat{\mathbf{b}}^{\dagger}-\hat{\mathbf{b}}^{\dagger} \hat{\mathbf{b}} \rho_{S}(t)-p_{S}(t) \hat{\mathbf{b}}^{\dagger} \hat{\mathbf{b}}\right) \\
& +k_{c}\left(2 \hat{a} \rho_{S}(t) \hat{b}^{\dagger}-\hat{\mathbf{a}} \hat{\mathbf{b}} \rho_{S}(t)-\rho_{S}(\hat{t}) \hat{a}^{\dagger} \hat{\mathbf{b}}\right)
\end{aligned}
$$

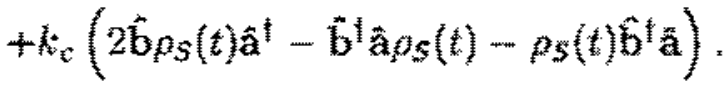

Da definição do superoperádor Liouviliano, 1.16 , e da equaçäo 1.17 obtemos o liouvilliano para um sistema de dois osciladores acoplados entre si, forçados externamente e em contato com um reservatório:

$$
\begin{aligned}
& \mathcal{L}=-\frac{i}{\hbar}\left[\mathbf{H}_{S^{\prime}}, \cdot\right] \\
& +k_{a}\left(2 \hat{\mathbf{a}} \cdot \hat{\mathbf{a}}^{\dagger}-\hat{\mathbf{a}}^{\dagger} \hat{\mathbf{a}} \cdot-\cdot \hat{\mathbf{a}}^{\dagger} \hat{\mathbf{a}}\right)+k_{\mathrm{b}}\left(2 \hat{\mathbf{b}} \cdot \hat{\mathbf{b}}^{\dagger}-\hat{\mathbf{b}} \hat{\mathrm{b}} \cdot \hat{\mathbf{b}}-\hat{\mathbf{b}}^{\dagger} \hat{\mathbf{b}}\right) \\
& +h_{c}\left(2 \overrightarrow{\mathbf{a}} \cdot \hat{\mathbf{b}}^{\dagger}-\hat{\mathbf{a}}^{\dagger} \hat{\mathbf{b}} \cdot-\hat{\mathbf{a}}^{\dagger} \hat{\mathbf{b}}\right)+k_{c}\left(2 \hat{\mathbf{b}} \cdot-\hat{\mathbf{b}}^{\dagger} \hat{\mathbf{a}} \cdot-\cdot \hat{\mathbf{b}}^{\dagger} \hat{\mathbf{a}}\right) .
\end{aligned}
$$

oul

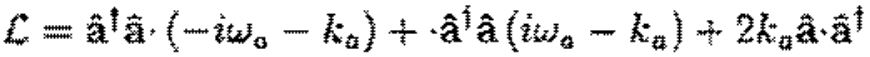

$$
\begin{aligned}
& +\hat{b} \hat{b} \cdot\left(-\hat{z} \omega_{b}-k_{b}\right)+\hat{b}+\hat{b}\left(w_{b}-k_{b}\right)+2 k_{b} \hat{b} \cdot \hat{b}^{t} \\
& +\hat{a}^{\dagger} \hat{\mathbf{b}} \cdot\left(-\hat{i} g-k_{c}\right)+\hat{a}^{t} \hat{\mathbf{b}}\left(\hat{b} g-k_{c}\right)+2 k_{c} \mathrm{a}_{\mathrm{a}} \mathbf{b} \\
& +\hat{a} \hat{b}^{\dagger} \cdot\left(-i g-k_{c}\right)+\hat{a}^{\hat{b}}\left(i g-k_{c}\right)+2 k_{c} \hat{b} \cdot \hat{a}^{t} \\
& -i\left[f_{\mathrm{a}} \hat{\mathrm{a}}^{t}+f_{\mathrm{a}}^{*} \mathrm{a}_{i} \cdot\right]-i\left[f_{b} \hat{\mathrm{b}}^{\dagger}+f_{b}^{*} \hat{\mathrm{b}}, \cdot\right]
\end{aligned}
$$

onde $k_{i}{ }^{2}=k_{b} k_{a}$.

A presença das fontes e do acoplamento entre os osciladores, que está contida dentro do hamiltoniano, $\mathbf{H}_{S^{\prime}}$, näo altera a estrutura da equação 4.11 pois, ao definirmos a relação 4.3 e fazermos a substituiçāo 4.10 , näo precisamos saber quem é $\mathbf{H}_{0}$ dado que a dependência tenporal de â( $(t)$ mantém plausivel as aproximaçós feitas pois ela $a$ lenta comparada com as escalas de tempo do reservatótio. Com isto, podemos escrever o superoperador Liouvilliano com ou sem fonte $f$, com ou sem acoplamento g, se cancelarmos na equação 4.13 os termos que năo nos interessam, sem comprometer a deduçăo da equaçă̄o Mestra. Assim podemas escreverer as hamiltonianas, 
$\mathbf{H}_{a}+\mathbf{H}_{b}+\mathbf{H}_{9}$, dos dois osciladores acoplados, em modos normais, por exemplo, e obter a equaçäo Mestra

$$
\begin{aligned}
& C=a_{1}^{+} \hat{a}_{1} \cdot\left(-i \omega_{1}-k_{1}\right)+\cdot a_{1}^{\dagger} \hat{a}_{1}\left(i \omega_{1}-k_{1}\right)+2 k_{1} \hat{a}_{1} \cdot a_{1}^{\dagger} \\
& +\hat{a}_{2} \hat{a}_{2 "}\left(-i w_{2}-k_{2}\right)+\cdot \hat{a}_{2}^{\dagger} \hat{a}_{2}\left(i w_{2}-k_{2}\right)+2 k_{2} \hat{a}_{2} \cdot \hat{a}_{2}^{\dagger} \\
& +\hat{a_{1}} \hat{a}_{2} \cdot\left(-k_{3}\right)+\cdot \hat{a}_{1}^{\dagger} \hat{a}_{2}\left(-k_{3}\right)+2 k_{3} \hat{a}_{1} \cdot \hat{a}_{2}^{\dagger} \\
& +\hat{a}_{1} a_{2} \cdot\left(-k_{3}\right)+\hat{a}_{1} \hat{a}_{2}^{\dagger}\left(-k_{3}\right)+2 k_{3} \hat{a}_{2} \cdot \hat{a}_{1}^{\dagger}
\end{aligned}
$$

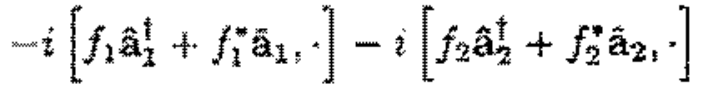

on

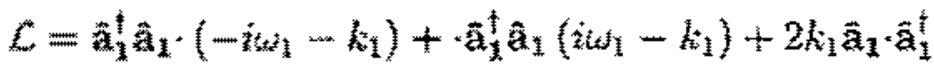

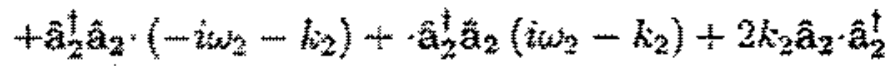

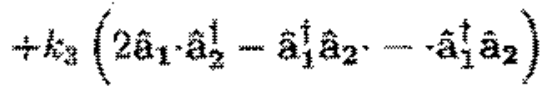

$$
\begin{aligned}
& +k_{3}\left(2 a_{2} \cdot a_{1}^{t}-\hat{a}_{1} a_{2}^{t}-\hat{a}_{1} \hat{a}_{2}\right) \\
& -i\left[f_{1} \hat{a}_{1}^{\dagger}+f_{1}^{*} \hat{a}_{1 *} \cdot\right]-i\left[f_{2} \hat{a}_{2}+f_{2}^{*} \hat{a}_{2}, \cdot\right]
\end{aligned}
$$

onde $h_{3}{ }^{2}=k_{2} h_{1}$.

Se os osciladores näo forem forçados, podemos simplesmente eliminar a fonte da equaçăo anterior e escrever:

$$
\begin{aligned}
& \mathcal{L}_{0}=\mathrm{a}_{1}^{\dagger} \hat{\mathbf{a}}_{1} \cdot\left(-i \omega_{1}-k_{1}\right)+\cdot \hat{\mathrm{a}}_{1}^{\dagger} \hat{\mathrm{a}}_{1}\left(i \omega_{1}-k_{1}\right)+2 k_{1} \hat{\mathrm{a}}_{1} \cdot \hat{\mathrm{a}}_{\mathrm{I}}^{\overline{1}} \\
& +a_{2}^{t} \vec{a}_{2} \cdot\left(-i \omega_{2}-k_{2}\right)+\hat{a}_{2}^{t} \hat{a}_{2}\left(i \omega_{2}-k_{2}\right)+2 k_{2} \hat{a}_{2} \cdot \hat{a_{2}} \\
& +k_{3}\left(2 \hat{a}_{1} \cdot \hat{a}_{2}^{\dagger}-\hat{a}_{1}^{\dagger} \tilde{a}_{2} \cdot-\cdot \hat{a}_{1}^{\dagger} \tilde{a}_{2}\right) \\
& +k_{3}\left(2 \hat{a}_{2} \cdot \hat{a}_{1}^{\dagger}-\mathbf{a}_{1} \hat{a}_{2}^{\dagger} \cdot-\hat{a}_{1} \hat{a}_{2}^{\dagger}\right)
\end{aligned}
$$

onde $k_{9}^{2}=k_{2} k_{1}$

Note que se fizermos, na equaçăo 4.13 , uma troca de índices e eliminarmos $g$ obteremos a equaçäo 4.14. Por outro lado, se escrevermos a equaçāo 4.13 em modos normais, obteremos estruturalmente a mesma equação anterior com diferença na redefiniçato dos $k s$, ou seja, neste caso:

$$
\begin{aligned}
& k_{1}=k_{a} h_{1}^{2}+k_{b} h_{2}^{2}+2 k_{c} h_{1} h_{2} \\
& k_{2}=k_{a} h_{2}^{2}+k_{b} h_{2}-2 k_{c} h_{1} h_{2} \\
& k_{3}=h_{1} h_{2}\left(k_{b}-k_{a}\right)+k_{c}\left(h_{1}^{2}-h_{2}^{2}\right)
\end{aligned}
$$


onde

$$
\begin{aligned}
& h_{1}=\sqrt{\frac{\Omega_{1}-\omega_{2}}{R}}, \quad \quad h_{2}=\sqrt{\frac{\Omega_{1}-\omega_{0}}{R}}, \\
& R=\sqrt{\left(w_{\mathrm{a}}-w_{b}\right)^{2}+4 g}, \quad \Omega_{z}=\frac{\omega_{a}+\omega_{k} \pm R}{2}, \\
& \overrightarrow{\mathrm{a}}=h_{1} \hat{\mathrm{a}}_{1}-h_{2} \hat{\mathrm{A}}_{2,}, \quad \hat{\mathrm{b}}=h_{1} \hat{\mathrm{a}}_{2}+h_{2} \hat{\mathrm{a}}_{1} \text {, } \\
& g \leq \sqrt{\psi_{a} \omega_{b}}
\end{aligned}
$$

Note que existe um limite superior físico para a intensidade da constante de acoplamento entre os osciladores. No limite para $g=\sqrt{w_{a^{2} w_{b}}}$ teremos $\Omega_{3}=0$ e para valores maiores uma instabilidade será gerada, pois um dos modos normais apresentarä freqüência negativa.

Podemos observar ainda que a existencia de $k_{c}$ (ou $k_{3}$ ) vem do fato dos osciladores estarem imersos no mesno banho, enquanto que $k_{3}^{\text {(mn) }}$ existe independente deste fato. Quando os osciladores näo estâo no mesmo banho, a hamiltoniana de interaçäo $\mathrm{H}_{\text {in }}$, se altera, alterando a estrutura da equação 4.11. Observando o desenvolvimento da equaçăo Mestra, verificamos que sendo os banhos diferentes cada oscilador terá sua hamiltoniana de interação fazendo com que os termos cruzados que geran $k_{t}$ (ou $k_{3}$ ), equaçäo 4.4 , deixem de existir, ou seja:

$$
\left(\Gamma_{h_{i}}(t) \Gamma_{h_{j}}^{\dagger}(t)\right)=0 \text { para } h_{i} \neq h_{i} \Longrightarrow \nexists k_{i}\left(\text { ou } k_{3}\right)
$$

\subsection{Operador densidade para dois osciladores}

Deduzida a equação Mestra, podemos calcular a evoluçäo ternporal de um estado inicial coerente fatoriado $\rho_{s}(0)=\left|\alpha_{1}\right|\left(\alpha_{1}|\otimes| \alpha_{2} \mid\left(\alpha_{2} \mid\right.\right.$ onde $\left.\mid \alpha_{1}\right),\left|\alpha_{2}\right\rangle$ sato estados dos osciladores representados pela hamiltoniana $H_{1}=h \Omega_{2}\left(\hat{a}_{1}^{\dagger} z_{2}+\frac{1}{2}\right)$. $\mathbf{H}_{2}=\hbar \Omega_{2}\left(\hat{a}_{2}^{l} \hat{\mathbf{a}}_{2}+\frac{1}{2}\right)$ respectivamente. Para obtermos a evoluçäo temporal, dada pela equaçăo 1.17, utilizaremos o ordenamento do liouvilliano sem fontes dado no apêndice $B .2$ e escreveremos ${ }^{1}$ :

$$
\begin{aligned}
& \underbrace{p(t) s} \equiv p_{0}(t) \\
& \text { (sem fantosit) }
\end{aligned}
$$

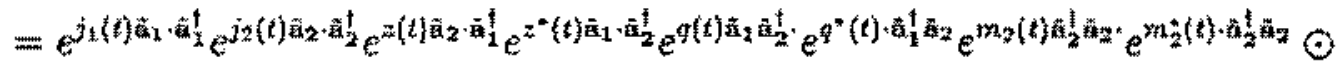

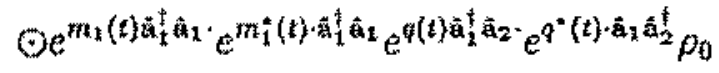

Para calcularmos os coeficientes $\left(q(t), m_{1}(t), m_{2}(t)\right.$ e $j_{1}\langle t), j_{2}(t), z(t)$, equaçōes B.2 e A.20 respectivamente) deste desacoplamento, utilizamos o sistema descrito pela

\footnotetext{
Toravante omitiremos o subscrito $S$.
} 
equação 4.15. Podemos entretanto utilizar o mesmo procedimento para calcular os coeficientes do sistema descrito pela equação $4.13 \mathrm{sem}$ fontes obtendo resultado semelhante ao encontrado. Assin, podemos generalizar os coeficientes escrevendo:

$$
\begin{aligned}
& q(t)=2 D\left(1-e^{r t}\right)\left(\Delta_{+} e^{r t}-\Delta_{-}\right)^{-1} \text { para } r \neq 0 \\
& e^{m_{2}(t)}=\frac{e^{-R t}}{2 r} e^{-\frac{r t}{2}}\left(\Delta_{+} e^{r t}-\Delta_{-}\right) \\
& e^{m m_{3} t h t}=e^{-2 R t} e^{-m_{t}(t)}
\end{aligned}
$$

onde

$$
\begin{aligned}
& r=\sqrt{c^{2}+4 D^{2}}, \quad c=k_{2}-k_{1}+i\left(\Omega_{2}-\Omega_{1}\right), \\
& R=\frac{k_{2}+k_{1}}{2}+\frac{i\left(\Omega_{2}+\Omega_{1}\right)}{2}, \quad \Delta_{(+)}=c\left(\begin{array}{c}
+ \\
-
\end{array}\right) r
\end{aligned}
$$$$
D=k_{3} \text { para o sistema descrito pala equação } 4.15
$$$$
D=k_{t}+i g \text { para o sistema descrito pala equação } 4.13 \text { sem fontes }
$$

$D=k_{i}$ paxa o sistema descrito pala equaçäo 4.13 sem fontes e desacoplado.

A atuação dos superoperadores, dados na equação 4.16 , sobre um estado coerente fol desenvolvida no apéndice A.3, e o resultado é dado pela equaçăo A.21

$$
\left.p(t)=\mid \alpha_{1 i}\right)\left(\alpha_{1 t}|\otimes| \alpha_{2 i}\right)\left(\alpha_{2 t} \mid\right.
$$

onde

$$
\left.\begin{array}{l}
\alpha_{t z} \equiv \alpha_{1} e^{m_{1}(t)}+\alpha_{2} q(t) e^{m_{2}(t)} \\
\alpha_{2 t} \equiv \alpha_{1} q(t) e^{m n_{2}(t)}+\alpha_{2}\left[e^{m_{2}(t)}+q(t)^{2} e^{m_{1}(t)}\right]
\end{array}\right\}
$$

De maneira análoga podemos escrever para o sistema descrito pela equação 4.13

$$
\rho(t)=\left|\alpha_{A t}\right\rangle\left\langle\alpha_{A t}|\otimes| \alpha_{B t}\right\rangle\left\langle\alpha_{B t}\right|
$$

onde

$$
\left.\begin{array}{l}
\alpha_{A t} \equiv \alpha_{A} e^{m_{1}(t)}+\alpha_{B} q(t) e^{m_{1}(t)} \\
\alpha_{B t} \equiv \alpha_{A} q(t) e^{m_{1}(t)}+\alpha_{B}\left[e^{m_{Z}(t)}+q(t)^{2} e^{m_{1}(t)}\right]
\end{array}\right\}
$$


e os coeficientes săo dados pelas condiçōes da equaçäo 4.17.

Podemos mostrar a simetria entre $\alpha_{1 t}$ e $\alpha_{2 t}$ partindo de um mesmo estado inicial $\alpha_{1}=\alpha_{2}=\alpha$

$$
\begin{aligned}
& \alpha_{1 t}=\alpha e^{m t_{1}(t)}(1+q(t)), \\
& \alpha_{2 t}=\alpha\left[e^{m_{2}(t)}+q(t) e^{m_{1}(t)}(1+q(t))\right] .
\end{aligned}
$$

fazendo as constantes de dissipação e as frequências iguais e utilizando a equação B.4, obtendo

$$
\alpha_{1 t}=\alpha_{2 t}=\alpha e^{-(i \omega+2 k t)}
$$

Alén de notarmos a simetria, como era esperado, vemos que os osciladores dissiparn energia com constantes de dissipação dobrada devido ao acoplamento entre eles via banho.

Una ou tra maneira de observarmos a simetria entre $\alpha_{1 t} \in \alpha_{2 t}$ é ordenarmos a equação 4.16 de forma a trocar "1" con "2". A evoluçāo temporal que encontraremos tem estrutura inversa à exposta na equaçăo 1,19 , com coeficientes diferentes.

A equaçäo 4.14 representa o mesmo sistema que acabamos de descrever, acrescida de fonte. Podemos calcular o estado coerente estacionário do sistema descrito pela equação 4.14 on do sistema descrito pela equaçăo 4.13 , sem conbecer sua evolução temporal, fazendo:

$$
\frac{d}{d t} \rho(t)=0 \quad \rightarrow \quad C \rho(0)=0
$$

e encontrar um produto de estados coerentes dado por:

$$
\rho_{\text {estat. }}=\left|\mathcal{A}_{A}\right\rangle\left\langle\mathcal{A}_{A}|\otimes| \mathcal{A}_{B}\right\rangle\left\langle\mathcal{A}_{2}\right|
$$

onde:

$1^{\circ}$ Se o sistema for descrito pela equação 4.14

$$
\mathcal{A}_{2}=\frac{i f_{2} k_{3}-i f_{1}\left(i_{2} w_{2}+k_{z}\right)}{-\omega_{1} \omega_{2}+i\left(\omega_{1} k_{2}+\omega_{2} k_{1}\right)}
$$

$2^{\circ}$ Se o sistema for descrito pela equaçăo 4.13

$$
A_{A}=\frac{i f_{B}\left(i g+k_{C}\right)-i f_{A}\left(i \omega_{B}+k_{B}\right)}{\left(g^{3}-\omega_{A} \omega_{B}\right)+i\left(\omega_{A} k_{B}+\omega_{B} k_{A}\right)}
$$

A condição necessária para zeray o denominador da equação 4.23 ou 4.24 é dada pela parte real do denominador igual a zero. Isto só ocorre quando tivermos uma partícula livre, ou seja, quando $\omega_{A}$ ou $\omega_{y}$ for igual a gero, conseqüentemente dada a condiçäo de energia positiva $0 \leq g^{2} \leq \omega_{A} \omega_{B} \rightarrow g=0$, ou ainda uma "partícula livre nos modos normais", pois se $g^{2}=\omega_{A} \omega_{b} \rightarrow \omega_{2}=0$. Para obtermos a 
evolução temporal de um dos sistemas com fonte, podemos utilizar o ordenamento dos superoperadores sem fontes, $\hat{C}_{0}$, acrescentando os superoperadores da fonte. Ao fazermos o desacoplamento, notamos que a presença dos superoperadores com fontes năo geram os superoperadores de $L_{0}$. Consequientemente, os coeficientes encontrados no desacoplamento de $\mathcal{L}_{0}$ permaneceräo os mesmos. Assim, podemos escrever o ordenamento partindo da evoluçẫo temporal $\rho_{0}(t)$ obtida na equaçăo 4.16 sem fontes ${ }^{2}$ e obter:

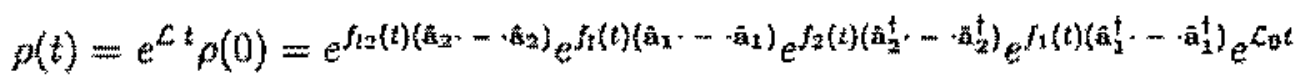

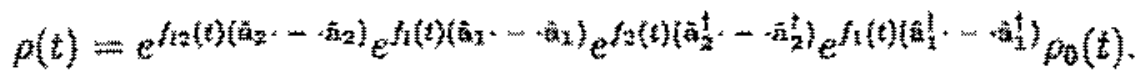

Utilizando a tetchica exponencial 3.2 .2 , detalhada no apêndice $B$, encontraremos um sistema de equaçòes cuja solução nos dará os coeficientes

$$
\begin{aligned}
& f_{1}(t)=-f_{1}^{*}(t) \quad f_{2}(t)=-f_{l 2}^{*}(t) \\
& \left(\begin{array}{l}
f_{1}(t) \\
f_{2}(t)
\end{array}\right)=\mathcal{A}_{2}\left\{1+\frac{e^{-\left(R-\frac{r}{2}\right) t}}{r}\left[e^{-r t}\left(R-\frac{T}{2}\right)-\left(R+\frac{r}{2}\right)\right]\right\} \\
& +\frac{i e^{-\left(n-\frac{n}{2}\right) t}}{r} f_{k}\left(e^{-s i}-1\right)
\end{aligned}
$$

onde $R, r$ e $\mathcal{A}_{1}$ sằo dados pelas equaçöes 4.17 e 4.23 respectivamente.

Com isto, podemos escrever a evolução temporal para um estado inicial produto de dois estados coerentes e descorrelacionados para o sistema descrito pela equação 4.14

$$
\rho(t)=\left|\alpha_{1} f\right|\left|\alpha_{1 f}\right| \otimes\left|\alpha_{2 f}\right|\left\langle\alpha_{2 f t}\right|
$$

onde

$$
\alpha_{\left(\frac{1}{2}\right) f t}=\alpha_{\left(\begin{array}{l}
1 \\
2
\end{array}\right) t}+f_{\frac{1}{2}}(t)
$$

e $\alpha_{\left(\begin{array}{l}1 \\ 2\end{array}\right)}, f_{\frac{1}{2}}(t)$ sẫo dados pelas equaçôes $4.19,4.26$ respectivamente.

A equaçâo Mestra leva para um estado que é assintoticamente independente do tempo, sem importar de qual estado se parta. Portanto, calcular a soluçăo geral como funçäo do tempo, $\rho(t)$ e tomar o limite assintótico é o mesmo que encontrar o estado estacionário calculado em 4.22 , ou seja,

$$
\lim _{t \rightarrow \infty} p(t)=\rho_{\text {estacs. }}
$$

\footnotetext{
${ }^{2}$ desenvolvimento vemelhante foi feito na seçäo 3.2 .2 .1
} 
De maneira análoga podenos calcular a evolução temporal para o sistema descrito pela equação 4.13 e escrever:

$$
p(t)=\left|\alpha_{A f t}\right\rangle\left\langle\alpha_{A f t}|\otimes| \alpha_{B f t}\right\rangle\left\langle\alpha_{B f t}\right|
$$

onde

$$
\begin{aligned}
& \alpha_{\left(\begin{array}{c}
A \\
a
\end{array}\right) f t}=\alpha_{(a) t}^{A}+f_{B}^{A}(t), \\
& \left(\begin{array}{l}
f_{A}(t) \\
f_{B}(t)
\end{array}\right)=A_{A}\left\{1+\frac{e^{-\left(R-\frac{r}{2}\right) t}}{r}\left[e^{m r t}\left(R-\frac{r}{2}\right)-\left(R+\frac{r}{2}\right)\right]\right\} \\
& +\frac{3 e^{-(R-r) t}}{r} f_{t}\left(e^{-r t}-1\right)
\end{aligned}
$$

e os coeficientes $R_{2} r$ e $A_{A}$ säo dados pelas equaçóes 4.17 e 4.24 respectivamente.

\subsection{Limite Assintótico - Aplicaçōes}

Encontrados os estados estacionários, equaçóes 4.23 e 4.24 , podemos calcular a excitação média, $\varepsilon$, para cada um dos osciladores e analizar as trocas de excitaçôes medidas pelos acoplamentos dos osciladores entre si e via banho. Considerando o sistema descrito pela equaçäo 4.14 obtemos

$$
\varepsilon_{2}=\operatorname{Tr}\left\{\left(\begin{array}{l}
\hat{a}_{1}^{\dagger} \hat{a}_{1} \\
\hat{a}_{2}^{\dagger} \hat{a}_{2}
\end{array}\right)\left(\begin{array}{l}
\left|\mathcal{A}_{1}\right\rangle\left\langle\mathcal{A}_{1}\right| \\
\left|\mathcal{A}_{2}\right\rangle\left\langle\mathcal{A}_{2}\right|
\end{array}\right)\right\}
$$

ou

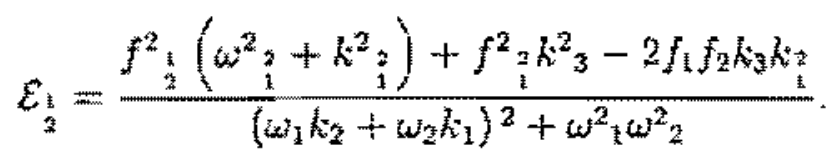

Analogamente para o sistenta descrito pela equação 4.13

$$
\mathcal{E}_{B}=\frac{f_{B}^{2}\left(\psi_{A}^{2}{ }_{A}+k_{A}^{2}\right)+f_{A}^{2} g^{2}+f_{A B}^{2} k_{C}^{2}-2 f_{A} f_{B}\left(k_{C} k_{A}+g w_{B}\right)}{\left(\omega_{A} k_{B}+\omega_{B} k_{A}-2 g k_{C}\right)^{2}+\left(g^{2}-k_{C}^{2}+k_{A} h_{B}-\omega_{A} \omega_{B}\right)^{2}}
$$

Calculando através da equação 4.29 , a excitação média para os osciladores acoplados via banho com a mesma constante de acoplamento, ou seja, $g=0$ $k_{1}=k_{2}=k_{3}=k$ iremos obter:

$$
\begin{aligned}
& \varepsilon_{3}=\frac{f^{2}{ }_{1} \omega^{2}{ }_{2}+k^{2}\left(f_{1}-f_{2}\right)^{2}}{k^{2}\left(\omega_{1}+\omega_{2}\right)^{2}+\omega^{2}{ }_{1} \omega^{2}{ }_{2}} \\
& \varepsilon_{2}=\frac{f^{2}{ }_{2} \omega^{2}{ }_{1}+k^{2}\left(f_{1}-f_{2}\right)^{2}}{k^{2}\left(\omega_{1}+\omega_{2}\right)^{2}+\omega^{2}{ }_{1} \omega^{2}{ }_{2}}
\end{aligned}
$$

Essas expressōes nos permite concluir que: 
- Se o bombeamento dos osciladores foren iguais, $f_{A}=f_{B}=f$, entäo terá maior excitação quem tiver menor frequêtncia.

- A diferença de excitaçäo entre os dois osciladores cresce com o quadrado da amplitude da fonte $f$.

- Quando as fontes forem diferentes e obedecerem a desigualdade

$$
\frac{f_{\left(w_{\text {mener }}\right)}}{w_{(\text {menor })}} \geq \frac{f_{\left(w_{\text {maior }}\right)}}{w_{\text {(maior })}}
$$

que ể una espécie de "fluxo de excitações", notamos que se o "fluxo de excitaçô" for o mesmo para os dois sistemas então o sistema de menor frequêtucia terá maior excitaçăo

As figuras 4.1 a,2 nos mostran que quem tem maior excitaçấ que tem menor frequêtncia, no caso oscilador 1 . Note nestas figuras que o que está prevalecendo $\mathrm{t}$ a desigualdade $f_{1} \omega_{2} \geq f_{2} \omega_{1}$ e não o fato do oscilador de nator excitaçāo estar sendo mais ou menos bombeado.

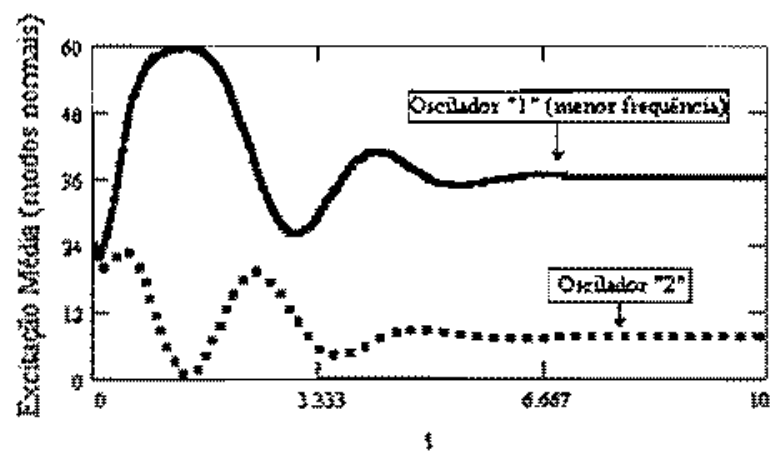

Figura 4.1: Comparando a excitaçâa média do oscilador 1 com 0 oscilador 2 onde $\omega_{1}=2$, $\mathrm{u}_{2}=3, f_{1}=15_{,} f_{2}=10, \mathrm{k}_{1}=\mathrm{h}_{2}=0.9$ 


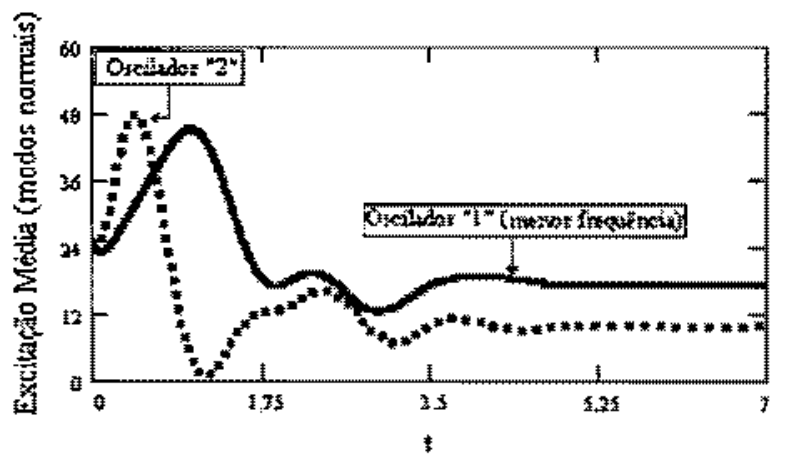

Figura 4.2: Comparando a excitaçu media do oseliador 1 com o oscilador 2 ande wi $=2$, $\omega_{2}=d_{3} f_{1}=10, f_{2}=15_{3} k_{1}=k_{2}=0.9$

Com o objetivo de tentar entender a diferença essencial entre acoplamentos "via g "e "via banho, $\mathrm{kc}_{\mathrm{f}}$ " comparamos a excitaçüo métia, $\varepsilon$, do oscilador "A" dissipando num banho $\varepsilon_{0}$

$$
\varepsilon_{A 0}=\frac{f_{A}^{2}\left(\omega_{B}^{2}+h_{B}^{2}\right)}{\left(\omega_{A} k_{B}+\omega_{B} h_{A}\right)^{2}+\left(k_{A} k_{B}-\omega_{A}\left(\omega_{B}\right)^{2}\right.}
$$

ou

$$
\varepsilon_{A 0}=\frac{f_{A}^{2}}{\omega_{A}^{2}+b_{A}^{2}}
$$

com:

- A excitaçäo média dele acoplado a "B" que díssipa em outro banho, $\mathcal{E}_{g}$. Devido ao contato com este segundo oscilador, o oscilador $A$ sofre dinâmicamente uma alteração na sua excitaçāo média. En particular, no regine estacionário, sua excitação média pode ser maior igual ou menor que o oscilador "A" desaco" plado do item anterior. Esta taxa é controlada essencialmente pela fonte do oscilador $B$.

Para escrever a excitaçäo média do oscilador $A$, usamos a equaçăo 4.30 e consideramos tic $=0$ obtendo:

$$
\varepsilon_{A g}=\frac{f_{A}^{2}\left(w_{B}^{2}+k_{B}^{2}\right)+f B g\left(f_{B} g-2 f_{A} w_{B}\right)}{\left(w_{A} k_{B}+w_{B} k_{A}\right)^{2}+\left(g^{2}+k_{A} k_{B}-w_{A} k_{B}\right)^{2}}
$$

on

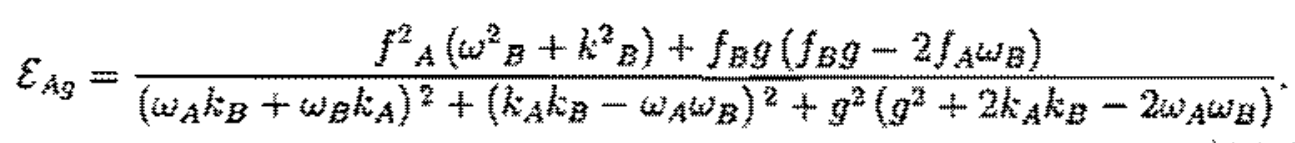

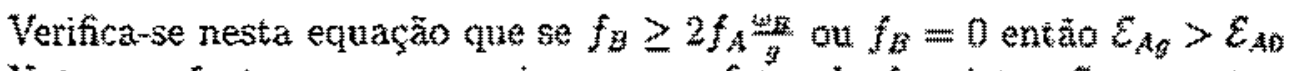
Note que $f_{B}$ tem que sar maior que um fator de $f_{A}$ isto nấo garante 0 fato de que: se $f_{B}>f_{A}$ então $\varepsilon_{A B}>\varepsilon_{A Q}$. Un outro fato curioso de se notar é que se o oscilador "B" não for bombeato, mesmo assim $\varepsilon_{A g}$ seri maior que $\varepsilon_{A a}$. 
- A excitação média dele acoplado a " $B$ " através da dissipação por um mesmo banho, $\varepsilon_{k}$. Neste caso, o acoplamento dos osciladores " $A$ e $B$ " será via banho. Para escrever a excitação média do oscilador A, usamos a equaçäo 4.30 e consideramos $g=0$ obtendo:

$$
\varepsilon_{A k}=\frac{f_{A}^{2}\left(\omega^{2} B+k_{B}^{2}\right)+f_{B} k_{C}\left(f_{B} k_{C}-2 f_{A} k_{B}\right)}{\left(\omega_{A} k_{B}+\omega_{B} k_{A}\right)^{2}+\left(k_{A} k_{B}-\omega_{A} \omega_{B}\right)^{2}+k_{C}^{2} C\left(k_{C}^{2}-2 k_{A} k_{B}+2 \omega_{A} \omega_{B}\right)^{2}} .
$$

A restriçăo imposta na deduçäo da equação Mestra, $k_{A} k_{B}<\omega_{A} \omega_{b}$ faz com que o denominador desta equaçāo seja maior que o da equaçāo 4.33 do primeiro item diffultando garantir as condiçöes de maior excitaçäo neếdia para $\mathcal{E}_{A k}$. $A$ garantia que podemos ter é a de que se $0 \leq f_{B} \leq 2 f_{A} \frac{k_{B}}{k_{E}}$ entäo $\varepsilon_{A k}<\varepsilon_{A 0}$.

Na figura 4.3 observamos que: o oscilador "A" tem maior excitaç⿰彳亍o quando acoplado "via g" e a amplitude de oscilaçāo da fonte " $\mathrm{B}$ " tor igutal a zero; existe uma faixa de amplitude de oscilação da fonte " $B$ " onde o oscilador "A"tem maior excitação se for acoplado "via $\mathrm{k}$; ; acoplamento "via $\mathrm{g}$ " $\mathrm{e}$ "via $\mathrm{k}$ "näo corresponde a soma deles individualmente.

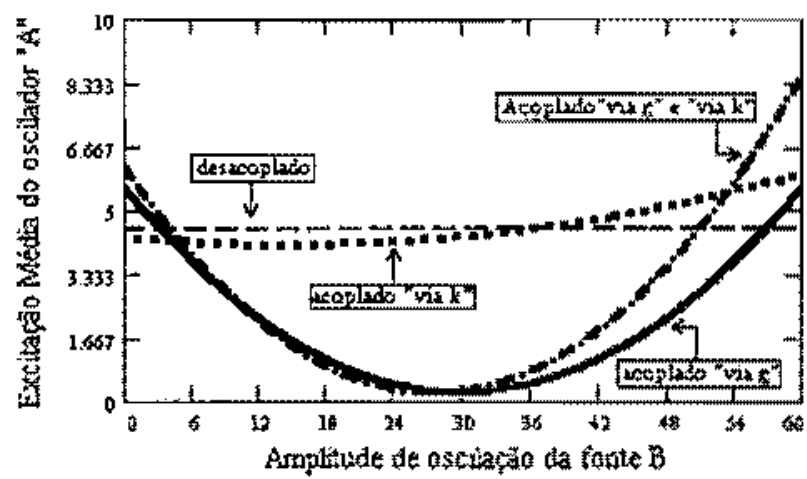

Figura 4.3: Comparando o oscilador " $\mathrm{A}$ " desacoplado, com ele acoplado de vários modos com ele desacoplado $\omega_{A}=7, \omega_{B}=4, f_{A}=15, k_{A}=k_{B}=0.9, g=2$

Para sabermos as condiços que leva a um dos osciladores "A ou $\mathrm{B}^{\text {" ter maior }}$ excitaçăo média consideramos um caso particular onde os dois osciladores estão acoplados e sendo bombeados, mas apenas o oscilador "A" dissipa num banho. Neste caso, para calcularmos a excitaçẫo média dada pela equaçăo 4,30, consideraremos $k_{B}=h_{C}=0$ e iremos obter:

$$
\begin{gathered}
\varepsilon_{A}=\frac{f_{A}^{2} b^{2} j^{2}+f^{2} B g^{2}-2 f_{A} f_{B} g \omega_{B}}{\left(\omega_{B} k_{A}\right)^{2}+\left(g^{2}-\omega_{A} \omega_{B}\right)^{2}} . \\
\mathcal{E}_{B}=\frac{f_{B}^{3}\left(\omega_{A}^{2}+k_{A}^{2}\right)+f_{A}^{2} g^{2}-2 f_{A} f_{B} g \omega_{A}}{\left(\omega_{B} k_{A}\right)^{2}+\left(g^{2}-\omega_{A} \omega_{B}\right)^{2}} .
\end{gathered}
$$


Veja que neste caso, a excitação média do oscilador "A"sempre diminuirá devido ao fato dele estar imerso num banho, enquanto que o oscilador " $B$ " poderá perder ou ganhar excitação média devido ao fato do oscilador "A" estar dissipando. Particularmente se as freqüências e os bombeamentos forem os mesmos, $\omega_{A}=\omega_{B}=\omega \mathrm{e}$ $f_{A}=f_{B}=f$ respectivamente a excitaçāo média do oscilador "B" será maior que a do oscilador "A" como era de se esperar. Por outro lado, se o oscilador "B" não for bombeado, $f_{B}=0$, e se $\omega_{(\text {menor })} \leq g \leq \omega_{\text {(maior) }}$, então terá maior energia quem tiver menor freqüência. A figura 4.4 mostra esta relaçāo e mostra também que existe uma faixa de amplitude de oscilaçāo da fonte " $\mathrm{B}$ " que faz o oscilador " $\mathrm{B}$ " ter menor excitação média.

Na figura 4.4 observamos que: o oscilador " $\mathrm{B}$ " poderá ter excitação média maior ou menor que a do oscilador "A" quando a amplitude da fonte "B" for zero, dependendo do valor de $g$; a freqüência do oscilador "A" determina se a excitação do oscilador " B" irá manter-se maior que a do oscilador " A" para qualquer freqüência.

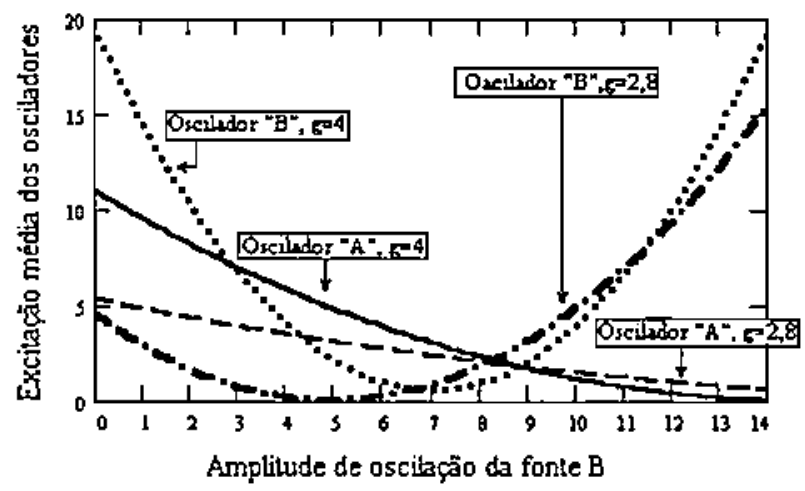

Figura 4.4: Comparando o oscilador "A" acoplado à um oscilador "B" via $g . \omega_{A}=11$, $\omega_{B}=3, f_{A}=20, k_{A}=2, k_{B}=0$

Tivemos a curiosidade de saber qual a probabilidade $P$ de num certo instante t, o estado estacionário ser atingido, ou seja,

$$
p=\operatorname{tr} \rho(t) \rho_{\infty}=e^{-\left|\alpha_{1 f t}-\mathcal{A}_{2}\right|^{2}}
$$

Dada a quantidade de parâmetros envolvidos tornando a expressão pouco elucidativa, optamos por elucidar graficamente. Observado na figura 4.5 podemos a princípio definir duas escalas de tempo; uma ligada a condição inicial e outra ligada a dinâmica propriamente dita. $O$ gráfico nos mostra também que o estado inicial $\alpha$ interfere na dinâmica do sistema. Constatamos em vários gráficos que fizemos que o bombeamento das fontes $f$ mantém por mais tempo a condição inicial enquanto que a dissipação $k$ leva rápidamente ao estado estacionário. 


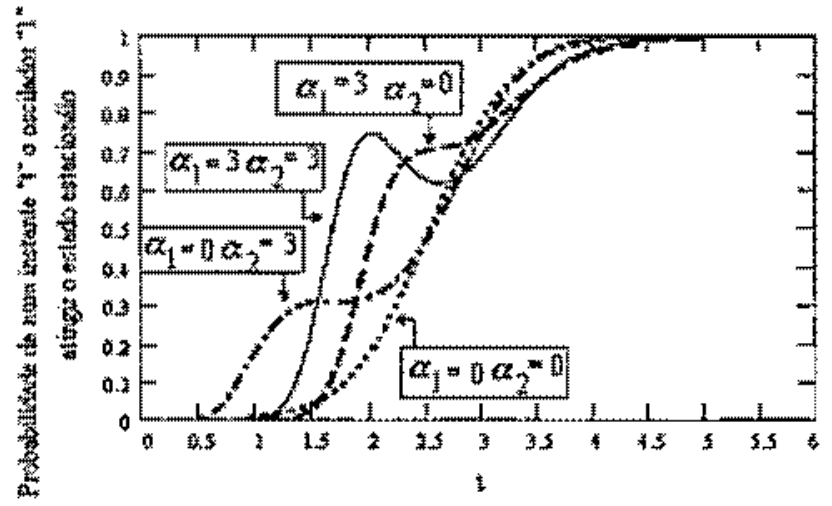

Figura 4.5: Comparando a excitaçäo media doo oscilador 1 com osciador 2 onde $w_{1}=2$, $\omega_{2}=4, f_{1}=10, f_{2}=15, h_{1}=h_{2}=0.9$

\subsection{Decoerência e Dissipação de uma Superpo- sição Coerente}

O fenomeno que iremos analisar é a dinàmica do entrelaçamento. Em todos os casos estudados com a condiçäo inicial de dois estados coerentes fatorizados, este fenomeno näo ocorreu. Partimos entäo de um estado que é constituido por uma superposiçăo coerente de estados coerentes en um dos osciladores e o outro num estado coerente fundamental. Vamos fazer uma aplicaçăo para estudar quantitativamente a relaçăo entre as escalas de tempo características da decoerencia e dissipaçāo ${ }^{3}$.

Seja

$$
\rho(0)=N^{2}(\alpha)(|\alpha\rangle+|-\alpha\rangle)(\langle\alpha|+\langle-\alpha|) \otimes \mid 0\rangle\langle 0|
$$

$\operatorname{com}$

$$
N(\alpha)=\left(2+2 e^{-2|\alpha|^{2}}\right)^{-\frac{1}{2}}
$$

A tuoluçăo temporal sem fontes dada pela equação 4.16 nos fornece

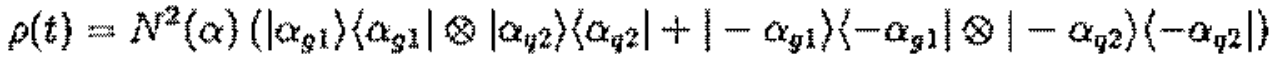

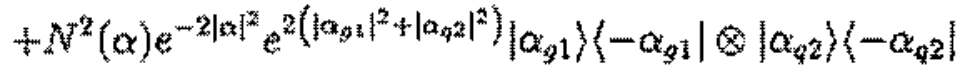

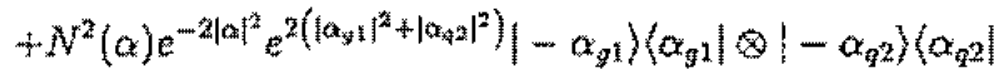

onde

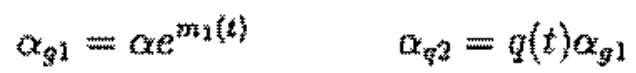

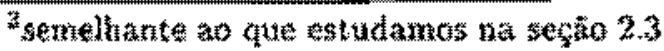


e os coeficientes $m_{1}(t), q(t)$ são dados pela equação 4.17 .

Para obtermos a evolução temporal para cada um dos osciladores faremos:

$$
\begin{aligned}
& \rho_{1}(t)=\operatorname{tr}_{2} \rho(t) \\
& \rho_{1}(t)=N^{2}(\alpha)\left(\left|\alpha_{g 1}\right\rangle\left\langle\alpha_{g !}|+|-\alpha_{g 1}\right\rangle\left\langle-\alpha_{g 1}\right|\right) \\
& \left.+N^{2}(\alpha) e^{-2|\alpha|^{2}} e^{2\left|\alpha_{g 1}\right|^{2}}\left(\mid \alpha_{g 1}\right)\left(-\alpha_{g 1}|+|-\alpha_{g 1}\right\rangle\left\langle\alpha_{g 1}\right|\right\}, \\
& p_{2}(t)=t_{1} \rho(t) \\
& \left.p_{2}(t)=N^{2}(\alpha)\left(\mid \alpha_{q_{2}}\right)\left\langle\alpha_{q^{2}}|+|-\alpha_{q}\right\rangle\left\langle-\alpha_{q_{2}}\right|\right) \\
& +N^{2}(\alpha) e^{-2|\alpha|^{2}} e^{2\left|\alpha_{q 2}\right|^{2}}\left(\mid \alpha_{q 2}\right)\left(-\alpha_{q 2}|+|-\alpha_{q 2}\right)\left(\alpha_{q 2} \mid\right)
\end{aligned}
$$

Como era de se esperar, ambos os osciladores dissipam para o reservatório e atingem seus estados fundamentais a tempos longos. Nesse processo, o oscilador 1 sofre a caracteristica decoerência de sua superposição inicial.

Note entretanto que há "transferéncia de coerência" ocasionada pelo acoplamento. $O$ oscilador 2, apesar de inicialmente sem superposiçoes, é levado a apresentar coerência entre estados distintos $\left|\alpha_{f 2}\right\rangle$ e $\left.\mid-\alpha_{12}\right\}$ e, a semelhança do oscilador 1 , tambén apresentará processo de decoerência. Una yez que os coeficientes, $m_{1}(t)$ e o(t), säo generalizados para vảrios sistemas de dois osciladores, entäo a evoluçăo temporal também pode representar a evolução de qualquer un dos sistentas apreseritados.

Podemos agora estudar a dissipação desses subsistemas, através do cálculo da energia.

$$
\begin{aligned}
& \mathrm{E}(t)=\operatorname{tr} \mathrm{H}_{\{2} \rho(t) \\
& \mathrm{E}(t)=2 \hbar^{2} \omega_{1} \omega_{2} N^{2}(\alpha)\left|\alpha_{g 1}\right|^{2}\left|\alpha_{q 2}\right|^{2}\left(1-e^{-2|\alpha|^{2}}\right) \\
& \mathrm{E}_{1}(t)=\operatorname{tr} \mathrm{H}_{1} \rho_{1}(t) \\
& \mathrm{E}_{1}(t)=2 \hbar \omega_{1} N^{2}(\alpha)\left|\alpha_{g 1}\right|^{2}\left(1-e^{-2|\alpha|^{2}}\right) \\
& \mathrm{E}_{2}(t)=t r \mathrm{H}_{2} \rho_{2}(t) \\
& \mathrm{E}_{2}(t)=2 \hbar \omega_{2} N^{2}(\alpha)\left|\alpha_{q 2}\right|^{2}\left(1-e^{-2|\alpha|^{2}}\right)
\end{aligned}
$$

E interessante observar que a razão entre as energias $E_{2}(t)$ e $E_{1}(t)$ em qualquer instante, resulta no módulo ao quadrado do coeficiente $|q(t)|^{2}$ que é diretamente proporcional à constante de "comunicação" entre os osciladores, acoplamento $g$ ou dissipação $k_{3}$ on $k_{c}$, dependendo do sistema utilizado.

Estudando a decoerência destes subsistemas através do defeito de idempotencia obtemos

$$
\delta(t)=1-\operatorname{tr} \rho^{2}(t)
$$




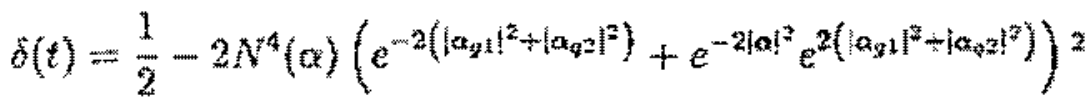

$$
\begin{aligned}
& \delta_{1}(t)=1-t+p^{2}{ }_{1}(t) \\
& \hat{\phi}_{1}(t)=\frac{1}{2}-2 N^{4}(\alpha)\left(e^{-2\left|\alpha_{g 1}\right|^{2}}+e^{-2|\alpha|^{2}} e^{2\left|\alpha_{g 1}\right|^{2}}\right)^{2} \\
& \delta_{2}(t)=1-\operatorname{tr} \rho_{2}^{2}(t) \\
& \delta_{2}(t)=\frac{1}{2}-2 N^{4}(\alpha)\left(e^{-2\left|\alpha_{\alpha}\right|^{2}}+e^{-2|\alpha|^{2}} e^{2 \mid \alpha_{q} !^{2}}\right)^{2} .
\end{aligned}
$$

Vamos definir os tempos caracteristicos de decoerência e dissipaçäo fazundo uma expansão em série de Taylor tomando os termos até primeira orkem para o oscilador cujo estado intcial é dado por una superposição de estados (no nosso caso to oscilador 1). Assim escreveremos para a energia e o defato de idempotencia, respectivamente:

$$
\begin{aligned}
& \mathrm{E}_{1}^{(n)}(t)=\left(\mathrm{E}_{1}(t)\right)_{t=0}+t\left(\frac{d}{d t} \mathrm{E}_{1}(t)\right)_{t=0} \\
& \mathrm{E}_{1}^{(t)}(t)=\mathrm{E}_{1}^{(0)}-t 4 k_{1}|\alpha|^{2} t_{\omega_{1}} N^{2}(\alpha)\left(1-e^{-2|\alpha|^{2}}\right) \\
& \delta_{1}^{(t)}(t)=\left(\delta_{1}(t)\right)_{t=0}+t\left(\frac{d}{d t} \bar{d}_{1}(t)\right)_{t=0} \\
& \delta_{1}^{(1)}(t)=t 8 k_{1}|\alpha|^{2} N^{2}(\alpha)\left(\lambda-e^{-2|\alpha|^{2}}\right)
\end{aligned}
$$

Definindo uma escala de tempo de dissipação

$$
-\frac{E_{1}^{(0)}-E_{1}^{(1)}(t)}{E_{\text {pto }}^{(0)}}=\frac{t}{\tau_{\text {dis: }}}
$$

onde

$$
T_{k=2}=\frac{4 k|\alpha|^{2} N^{2}(\alpha)\left(1-e^{-2|\alpha|^{3}}\right)}{|\alpha|^{2}+\frac{1}{2}}
$$

Analogamenta definimos

$$
\delta_{1}^{(1)}(t)=\frac{t}{\tau_{d e c}}
$$

$\operatorname{com}$

$$
{ }^{2}{ }_{\text {dec }}^{-1}=8 k_{1}|\alpha|^{2} N^{2}(\alpha)\left(1-e^{-2|\alpha|^{2}}\right)
$$

Uma vez garantido que o oscilador cujo estado inicial é dado por uma superposiçào de estados esta imerso em um banho, $k_{1} \neq 0$, então podemos fazer a relaçäo entre estes tempos

$$
\frac{\tau_{\text {diss }}}{\tau_{\text {dec }}}=2|\alpha|^{2}+1
$$


e obter curiosamente o mesmo resultado obtido na seção 2.3 .

Voltamos a modelo de dois osciladores acoplados dissipando en banhos diferentes, fizemos uma expansăo para o defeito de idempotencia do sistema " 1 "em $\frac{k}{k 2}$ e em $\frac{\mathrm{kl}}{\mathrm{k} 2}$ e ericontramos

$$
\delta_{1}^{(1)}(t)=8 t|\alpha|^{2} N^{2}(\alpha)\left(1-e^{-2|\alpha|^{2}}\right)\left(k 1+\frac{g^{2}}{k 2+\frac{\left(\omega_{2}-\omega_{1}\right)^{2}}{k^{2}}}\right)
$$

onde concluimos que quanto maior o acoplamento do sistema "2", $k_{2}$, ou quanto maior a diferença de oscilaçoes dos sistemas $\alpha_{z}-\omega_{1}$, menor a decoerencia. Fu partícular se 0 sistema " 1 "näo estiver acoplato, $h_{1}=0$, então

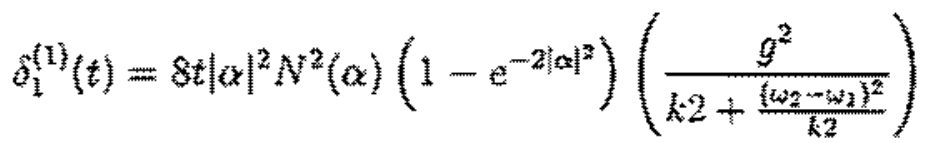

quanto maior o acoplamento menor a decoerência. 


\section{Conclusão}

Este trabalho e dedicado em parte ao desenvolvimento de ferramentas teóricas que permitem tratar decoerência e dissipaçăo de sistemas acoplados. Para tempos curtos comparados com os tempos característicos da evolugäo livre dos subsistemas, mos" tramos que enbora esses dois fenỏmenos tenham ingredientes comuns, são profundamente distintos. Utilizamos essa técnica perturbativa para explicar a "emergência da classicidade" en situaçoes que envolvem superposiçōes de estados quânticos. Introduaimos escalas de tempo características desses fenômenos que podem ser usadas facilmente em outros contextos.

Estudamos também fenômenos essencialmente vinculados a mais do que dois sistemas em interaçảo. Para isto, investimos en construir técnicas para calcular a densidade reduzida em tasos que sua dinâmica é suposta linerar, markoviana e obedeça aos critérios do teorema de Lindblad. Essas tếcnicas envolvem álgebra de Lie para superoperadores e foram usadas por $[14]$, dentre outros.

$O$ que inovamos no capítulo 3 e no apẻndice $B$ foi aplicar as técnicas diretamente para o cálculo do estado e nāo da funçāo de correlação associadas como havia sído feito anteriormente.

Em posse das técnicas apropriadas, calculamos a evolução temporal para vário tipos de sistemas. Calculamos a densidade total do sistema como função do tempo para a condiģǟo inicial de un produto de estados coerentes, fizemos aplicaçoes para observar propriedades do estado assintótico mostrando que existem comportamentos surpreendentes advindos desta dinâmica, como por exemplo o fato de uma fonte externa ser maior para um dos sistemas, nä̃o significar que este tẹá maior energia. Tendo cono motivaçäo principal un trabalho de H. Fröhlich que prevê comportamentos näo usuais e bastante interessantes para sistemas biológicos enwolvendo moléculas com momento dipolar que podem trocar energia através do reservatónio térmico no qual estāo imersas, consideramos duas moleculas representadas por dots osciladores em modos normais dissipando num mesmo reservatório com mesna constante de dissipaçäo e mostramos que mesmo reste caso simples, comportamentos näo usuais ao senso comum também ocorrem, como o fato de ter maior energia quem tiver menor fraquência mesmo que este esteja sendo bombeado com menor intensidade que o outro. Guatdada as devidas proporçöes, este resultado foi encontrado no trabalho de $\mathrm{H}$. Fröhlich o que nos motiva muito a buscar um modelo teórico mais próximo no modelo experimental proposto por ale.

Finalmente verificamos a dinâmica do entrelaçamento que ocorre com os sub sistema quando o stado inicial de un deles ê correlacionado. Definimos, atravts 
de uma expansảo, e calculamos os tempos de dissipaçäo e decoerễncia como feito no capitulo 2 para tempos curtos e surpeendentemente encontramos a mesma razão entre os tempos encontrada no capitulo 2, o que nos leva a crer que para o estado inicial dado, a razão entre os tempos de dissipaçăo e decoerência năo é afetada pela presença do outro oscilador nem do acoplamento entre eles. Fizemos uma expansäo para o defeito de idempotencia do sistema " 1 "em $\frac{8}{k 2} \mathrm{e} e \mathrm{kl} \frac{\mathrm{kl}}{\mathrm{k}}$ para um modelo de dois osciladores acoplados e dissipando em banhos diferentes concluimos que se aumentarmos o acoplamento do outro sistema, ou se aumentarmos a diferença de oscilação dos sistemas $\omega_{2}-\omega_{1}$, poderemos "segurar" a decoerêneia. 


\section{Apêndice A}

\section{Evolução de um estado coerente}

\section{A.1 Estados coerentes}

Um estado coerente é um estado de incerteza mínima. Tais estados são autoestados do operador de destruiçāo â, que nāo é auto-adjunto, tendo autovalores complexos. $O$ estado coerente do oscilador harmônico simples, $|\alpha\rangle$, pode ser expresso em termos do operador deslocamento de Glauber, 3.9, atuando no estado de vácuo $|0\rangle$, ou seja,

$$
\begin{aligned}
|\alpha\rangle & =D(\alpha)|0\rangle=e^{-\frac{2}{2}|\alpha|^{2}} e^{\alpha \overline{\mathbf{u}}_{1}^{\dagger}} e^{-\alpha^{*} \overline{\mathbf{a}}}|0\rangle \\
& =e^{-\frac{1}{2}|\alpha|^{2}} e^{\alpha \overline{\mathbf{a}}^{\dagger}} \sum_{n=0}^{\infty} \frac{\left(-\alpha^{*} \hat{\mathbf{a}}\right)^{n}}{n !}|0\rangle \\
& =e^{-\frac{1}{2}|\alpha|^{2}} e^{\alpha \hat{\mathbf{a}}^{\dagger}}\left(1-\alpha^{*} \hat{\mathbf{a}}+\ldots\right)|0\rangle \\
& \downarrow \\
|\alpha\rangle & =e^{-\frac{1}{2}|\alpha|^{2}} e^{\alpha \overline{\mathbf{a}}^{\dagger}}|0\rangle \longrightarrow\langle\alpha|=e^{-\frac{1}{2}|\alpha|^{2}}\langle 0| e^{\alpha^{*} \hat{\mathbf{a}}} \\
& \downarrow \\
|\alpha\rangle & =e^{-\frac{1}{2}|\alpha|^{2}} \sum_{n=0}^{\infty} \frac{\alpha^{n}}{n !}\left(\hat{\mathbf{a}}^{\dagger}\right)^{n}|0\rangle=e^{-\frac{1}{2}|\alpha|^{2}} \sum_{n=0}^{\infty} \frac{\alpha^{n}}{\sqrt{n !}}|n\rangle
\end{aligned}
$$

ou ainda

$$
\begin{aligned}
|\alpha+\beta\rangle & =e^{-\frac{1}{2}|\alpha+\beta|^{2}} e^{(\alpha+\beta) \overline{\mathbf{a}}^{\dagger}}|0\rangle=e^{-\frac{1}{2}|\alpha+\beta|^{2}} e^{\beta \overline{\mathbf{a}}^{\dagger}} \overbrace{}^{e^{\frac{1}{2}|\alpha|^{2}}|\alpha\rangle} \\
|\alpha+\beta\rangle & =e^{\frac{|\alpha|^{2}}{2}} e^{-\frac{1}{2}|\alpha+\beta|^{2}} e^{\beta \overline{\mathbf{a}}^{\dagger}}|\alpha\rangle \\
& \downarrow \\
e^{\beta \overline{\mathbf{a}}^{\dagger}}|\alpha\rangle & =e^{-\frac{|\alpha|^{2}}{2}} e^{\frac{1}{2}|\alpha+\beta|^{2}}|\alpha+\beta\rangle \\
& \downarrow \\
\langle\alpha| e^{\beta \overline{\mathbf{a}}} & =e^{-\frac{|\alpha|^{2}}{2}} e^{\frac{2}{2}|\alpha+\beta|^{2}}\langle\alpha+\beta|,
\end{aligned}
$$


mostrando que os estados coerentes nāo são auto estado de ${ }^{\dagger}$.

Para mostrarmos que $|\alpha\rangle$ é auto estado de â, faremos o seguinte cálculo:

$$
\begin{aligned}
\hat{\mathbf{a}} D(\alpha) & =\hat{\mathbf{a}}\left(e^{\alpha \mathbf{a}^{\dagger}} e^{-\frac{1}{2}|\alpha|^{2}-\alpha^{*} \overline{\mathbf{a}}}\right) \equiv e^{\alpha \hat{\mathbf{a}}^{\dagger}} f(\hat{\mathbf{a}}) \\
& =\hat{\mathbf{a}} \sum_{n=0}^{\infty} \frac{\alpha^{n}}{n !}\left(\hat{\mathbf{a}}^{\dagger}\right)^{n} f(\hat{\mathbf{a}})=\sum_{\mathbf{n}=\mathbf{b}}^{\infty} \frac{\alpha^{n}}{n !} \hat{\mathbf{a}}\left(\hat{\mathbf{a}}^{\dagger}\right)^{n} f(\hat{\mathbf{a}})
\end{aligned}
$$

como

$$
\begin{aligned}
& {\left[\hat{a}_{3}\left(\hat{a}^{t}\right)^{n}\right]=\left[\hat{a}_{i} \hat{a}^{n}\right]\left(\hat{a}^{\dagger}\right)^{n-1}+\hat{a}^{\dagger}\left[\hat{a}_{,}\left(\hat{a}^{t}\right)^{n-1}\right]} \\
& =\left[\hat{a}, \hat{a}^{y}\right]\left(\hat{a}^{1}\right)^{n-1}+\hat{a}^{t}\left[\hat{a}, \hat{a}^{\dagger}\right]\left(\hat{a}^{\dagger}\right)^{n-2}+\left(\hat{a}^{\dagger}\right)^{2}\left[\hat{a}_{i}\left(\hat{a}^{\dagger}\right)^{n-2}\right]=\ldots \\
& \ldots=n\left(\hat{a}^{i}\right)^{n-1}=\hat{a}\left(\hat{a}^{t}\right)^{n}-\left(\hat{a}^{n}\right)^{n} \hat{a}
\end{aligned}
$$

entāo

$$
\begin{aligned}
& \hat{a} D(\alpha)=\sum_{n=0}^{\infty} \frac{\alpha^{n}}{n !}\left(\hat{a}^{i}\right)^{n} a f(\hat{a})+\alpha \sum_{n=1=0}^{\infty} \frac{\alpha^{n-1}}{(n-1) !}\left(\hat{a}^{n}\right)^{n-1} f(\hat{a}) \\
& =D(\alpha) \hat{a}+\alpha D(\alpha)=D(\alpha)(\hat{a}+\alpha) \\
& \Downarrow \\
& D^{\dagger}(\alpha) \hat{a} D(\alpha)=\hat{\mathbf{a}}+\alpha \\
& \Downarrow \\
& D^{\dagger}(\alpha) \hat{\mathbf{a}}|\alpha\rangle=D^{\dagger}(\alpha) \hat{\mathbf{a}} D(\alpha)|0\rangle=(\hat{\mathbf{a}}+\alpha)|0\rangle=\alpha|0\rangle \\
& \text { 釉 } \\
& D(\alpha) D^{\dagger}(\alpha) \hat{a}|\alpha\rangle=D(\alpha) \alpha|\hat{0}\rangle \\
& \downarrow \\
& \hat{a}|\alpha\rangle=\alpha|\alpha\rangle=\langle\alpha| \hat{a}^{\dagger}=\langle\alpha| \alpha^{*}
\end{aligned}
$$

De maneira similar, demonstra-se que

$$
D^{\dagger}(\alpha) \hat{\mathbf{a}}^{\dagger} D(\alpha)=\hat{\mathbf{a}}^{\dagger}+\alpha^{*} .
$$

Para verificarmos a ortogonalidade dos estados coerentes faremos o produto escalar de dois estados coerentes $\{\alpha\rangle$ e $|\beta|$ expandindo numa base de numero, i é:

$$
\begin{aligned}
& \langle\beta \mid \alpha\rangle=e^{-\frac{1}{2}\left(|\alpha|^{2}+|\beta|^{2}\right)} \sum_{n, m}\left\langle m\left|\left(\frac{\beta^{m}}{\sqrt{m !}}\right)^{*} \frac{\alpha^{n}}{\sqrt{n !}}\right| n\right\rangle=e^{-\frac{1}{2}\left(|\alpha|^{2}+|\beta|^{2}\right)} \sum_{n, m} \frac{\alpha^{n} \beta^{* n n}}{\sqrt{n ! m !}} \delta_{n m n} \\
& \langle\beta \mid \alpha\rangle=e^{-\frac{1}{2}\left(|\alpha|^{2}+|\beta|^{2}\right)} \sum_{n} \frac{\alpha \beta^{* n}}{n !}=e^{-\frac{1}{2}\left(|\alpha|^{2}-2 \alpha \beta^{*}+|\beta|^{2}\right)} \neq \delta_{\alpha \beta}
\end{aligned}
$$

mostrando assin que a base dos estados coerentes nạo é ortonormal. 


\section{A.2 Superoperadores agindo em um estado coe- rente}

Do produto bilinear, 3.4, podemos agrupar a atuação das exponenciais dos superoperadores dados pela equaçäo 4.16 , de três maneiras:

- $1^{2}$ Maneira - Superoperadores "tipo 1-2" com ponto à direita ou à esquerda. A exponencial desses superoperadores atuando num estado coerente desloca o estado coerente. Detalharemos a seguir os cálculos para os quatro superoperadores que aparecem na equação em questão, da segunte forma:

1. Superoperator $\hat{a}_{1}^{\dagger} \hat{a}_{2}$.

$$
\left.e^{\left.q(t) \hat{A}_{1} \hat{A}_{2} \cdot \mid \alpha_{1}\right) \otimes\left|\alpha_{z}\right|}=\sum_{k} \frac{\left(q(t) a_{1}^{t} \hat{a}_{2}\right)^{k}}{k !} \mid \alpha_{1}\right) \otimes\left|\alpha_{2}\right|
$$

onde exprestamos a exponencial na forma de soma.

Uma vez que $\left[\hat{a}_{1}, \vec{a}_{2}^{*}\right]=0$, equação 3.4 , podemos separar $\hat{a}_{1}^{1}$ de $\hat{a}_{2}$ e escrever

$$
e^{q(t) \hat{\mathbf{a}}_{1}^{\dagger} \tilde{\mathbf{n}}_{2}}\left|\alpha_{1}\right\rangle \otimes\left|\alpha_{2}\right\rangle=\sum_{k} \frac{\left(q(t) \hat{\mathbf{a}}_{1}^{t}\right)^{k}}{k !}\left(\hat{a}_{2} \cdot\right)^{k}\left|\alpha_{1}\right\rangle \otimes\left|\alpha_{2}\right\rangle
$$

Como o estado coerente $\left|\alpha_{2}\right\rangle$ é auto estado do operador $\hat{\mathbf{a}}_{2}$, entầo

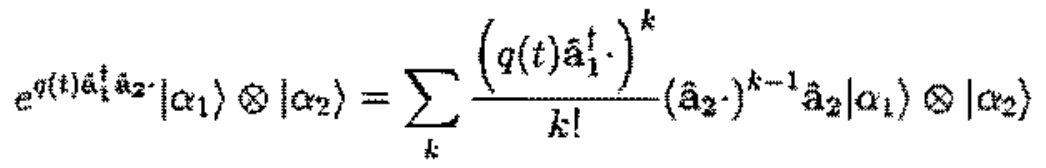

$$
\begin{aligned}
& \left.=\sum_{k} \frac{\left(q(t) \hat{a}_{1}^{\dagger}\right)^{k}}{k !} \alpha_{z}\left(\hat{a}_{2} \cdot\right)^{k-a_{2}} \mid \alpha_{1}\right) \otimes\left|\alpha_{2}\right\rangle \\
& \left.\left.=\sum_{k} \frac{\left(q(t) \hat{a}_{1}^{\dagger} \cdot\right)^{k}}{h !} \alpha_{2}^{k} \mid \alpha_{1}\right) \otimes \mid \alpha_{2}\right)
\end{aligned}
$$

retornando à forma exponencial

$$
e^{q(t) \hat{\hat{a}_{1}} \hat{\mathrm{a}}_{2} \cdot}\left|\alpha_{1}\right\rangle \otimes\left|\alpha_{2}\right\rangle=e^{g(t) \alpha_{2} \hat{\mathbf{a}}_{1}^{\prime}} \cdot\left|\alpha_{1}\right\rangle \otimes\left|\alpha_{2}\right\rangle
$$

Por outro lado, da equaçäo A.3 podemos concluir que:

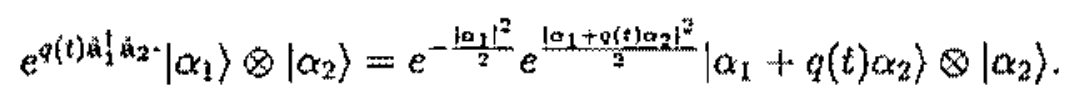


2. Superoperador $\hat{\mathbf{a}}_{2} \hat{\mathrm{a}}_{2}^{\mathrm{t}}$.

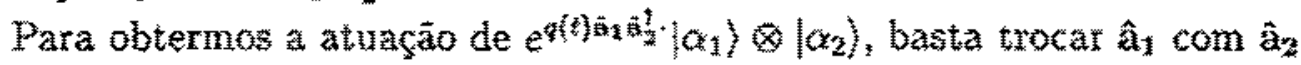
e $\alpha_{1} \operatorname{com} \alpha_{2}$ do item anterior, i $e$;

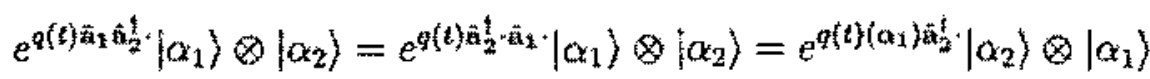

$$
\begin{aligned}
& \downarrow
\end{aligned}
$$

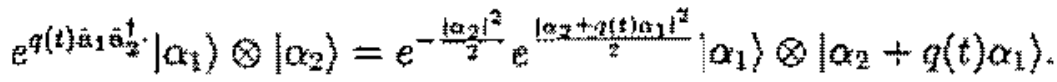

3. Superoperador $\cdot \mathbf{a}_{1} \mathrm{a}_{2}^{\dagger}$

$$
\begin{aligned}
& e^{q^{*}(t) \cdot \vec{a}_{z} \vec{a}_{2}^{t}}\left\langle\alpha_{1}\right| \otimes\left\langle\alpha_{2}\right|=\sum_{k} \frac{\left(q^{*}(\hat{t}) \cdot \hat{a}_{1} \hat{a}_{2}^{t}\right)^{k}}{k !}\left\langle\alpha_{1}\right| \otimes\left\langle\alpha_{2}\right| \\
& =\sum_{k}\left(\alpha_{1}\right) \otimes\left(\alpha_{2} \mid\left(\cdot \hat{a}_{2}\right)^{k} \frac{\left(q^{*}(t) \cdot \hat{a}_{1}\right)^{k}}{k !}\right. \\
& =\sum_{k}\left(\alpha_{1} \mid \otimes\left(\alpha_{2} \mid \hat{a}_{2}\left(\hat{a}_{2}^{t}\right)^{k-1} \frac{\left(q^{*}(t) \cdot \hat{a}_{2}\right)^{k}}{k !}\right.\right. \\
& =\sum_{k}\left\langle\alpha_{1}\right| \otimes\left\langle\alpha_{2}\right| \hat{\mathbf{a}}_{2}^{\dagger}\left(\cdot \hat{\mathbf{a}}_{2}^{\dagger}\right)^{k-2} \alpha_{2} \cdot \frac{\left(\hat{q}^{*}(\hat{t}) \cdot \hat{\mathbf{a}}_{1}\right)^{k}}{k !} \\
& =\left\langle\alpha_{1}\right| \otimes\left\langle\alpha_{2}\right| \sum_{k} \alpha_{2}{ }^{* k} \frac{\left(q^{*}(t) \cdot \hat{a}_{1}\right)^{k}}{k !}
\end{aligned}
$$

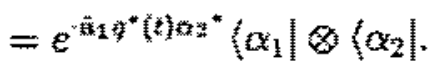

Da equaçào A.4 concluimos

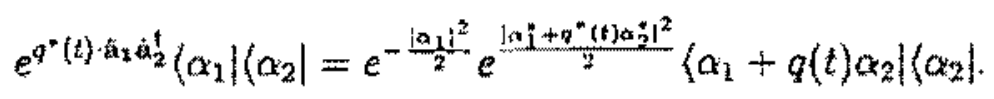

\section{Superoperador $\cdot \hat{a}_{2}$}

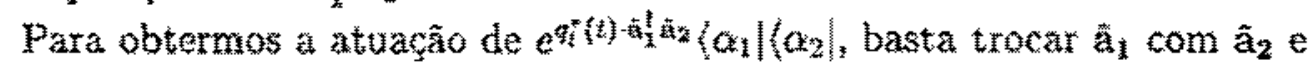
$\alpha_{1} \operatorname{com} \alpha_{2}$ do item anterior, i e:

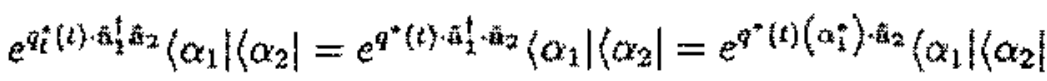

$$
\begin{aligned}
& \downarrow
\end{aligned}
$$

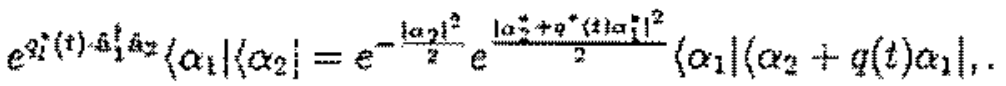

- $2^{\alpha}$ Maneira-Superoperadores "tipo 11"ou "tipo 22" com ponto à direita ou à esquerda. Para mostra que a exponencial desses superoperadores atuando num estado coerente, "gira" o estado e cria uma fase faremos os cálculos para os quatros superoperadores que aparecem na equaçäo 4.16 . 
1. Superoperadores $\hat{a}_{1}^{\dagger} \hat{\mathbf{a}}_{1}$. e $\hat{a}_{2}^{\dagger} \hat{a}_{2}$.

$$
\begin{aligned}
& e^{m_{1}(l) \hat{H}_{1} \tilde{n}_{\hat{k}_{2}}}\left|\alpha_{1}\right\rangle=\sum_{n} e^{m_{1}(t) \eta}|n\rangle\left\langle n|| \alpha_{1}\right\rangle \\
& =\sum_{n, n^{\prime}} e^{m_{1}(t) n}|n\rangle\left\langle n \mid n^{n}\right\rangle e^{-\left.\frac{1}{2} \alpha_{1}\right|^{2}} \frac{\alpha_{1}^{n^{\prime}}}{\sqrt{n^{\prime} !}} \\
& \left.=e^{-\frac{3}{2}\left|\alpha_{2}\right|^{2}} \sum_{n} e^{n n_{1}(t) n} \frac{\alpha_{1}^{n}}{\sqrt{n !}} \mid n\right)=e^{-\frac{1}{2}\left|\alpha_{1}\right|^{*}} \sum_{n} \frac{\left(\alpha_{1} e^{m_{1}(t)}\right)^{n}}{\sqrt{n !}}|n\rangle
\end{aligned}
$$

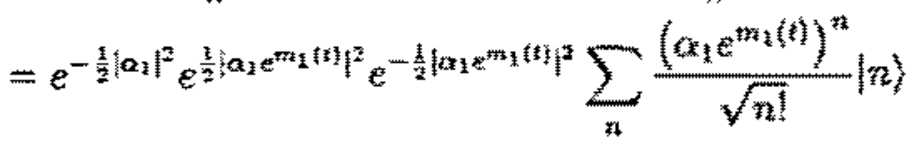

como

$$
\left.\left|\alpha_{1} e^{m_{1}(t)}\right\rangle=e^{-\frac{1}{2}\left|\alpha_{1} e^{m_{1}(t)}\right|^{2}} \sum_{n} \frac{\left(\alpha_{1} e^{m_{1}(t)}\right)^{n}}{\sqrt{n !}} \mid n\right)
$$

então podemos concluir

$$
e^{m_{x}(t) \mathrm{a}_{1}^{\dagger} \bar{a}_{1}} \cdot\left|\alpha_{1}\right\rangle=e^{-\frac{1}{2}\left|\alpha_{2}\right|^{2}} e^{\frac{1}{2}\left[\left.\epsilon^{m_{1}(t)} \alpha_{1}\right|^{2}\right.}\left|\alpha_{2} e^{m_{Y}(t)}\right\rangle
$$

Analogamente

$$
e^{r m_{2}(t) \hat{a}_{2}^{\dagger} \hat{A}_{2}} \cdot\left|\alpha_{2}\right\rangle=e^{-\frac{1}{2}\left|\alpha_{2}\right|^{2}} e^{\frac{1}{2}\left|e^{m m_{2}(t)} \alpha_{2}\right|^{2}}\left|\alpha_{2} e^{m m_{2}(t)}\right\rangle
$$

2. Superoperadores $\cdot \hat{\mathbf{a}}_{1}^{\dagger} \hat{\mathbf{a}}_{1} \mathrm{e} \cdot \hat{\mathbf{a}}_{2}^{\dagger} \hat{\mathbf{a}}_{2}$

$$
\begin{aligned}
& e^{m_{1}^{*}(t) \cdot \vec{a}_{1}^{t} \hat{a}_{1}}\left\langle\alpha_{1}\right|= \\
& =\sum_{n} e^{m_{i}(t) \pi^{*}}|n\rangle\left\langle n||\left|\alpha_{1}\right|\right.
\end{aligned}
$$

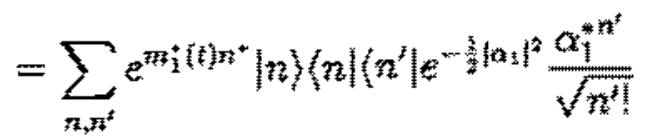

$$
\begin{aligned}
& =e^{-\frac{1}{2}\left|\alpha_{1}\right|^{2}} \sum_{n} e^{m i(t) n} \frac{\alpha_{1}^{* n}}{\sqrt{n !}} h_{i n} \mid \\
& =e^{-\frac{3}{2}\left|\alpha_{1}\right|^{2}} \sum_{n} \frac{\left(\alpha_{1}^{*} e^{m_{i}^{*}(t)}\right)^{n}}{\sqrt{n !}}\langle n|
\end{aligned}
$$

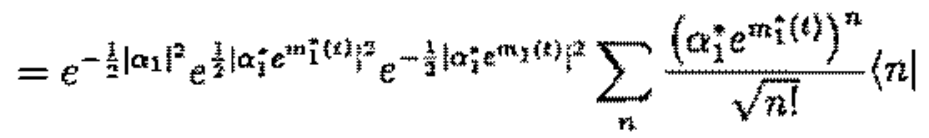

como

$$
\left\langle\alpha_{1} e^{m_{1}(t)}\right|=e^{-\frac{1}{2}\left|\alpha_{1} e^{m_{1}(t)}\right|^{2}} \sum_{n} \frac{\left(\alpha_{1}^{*} e^{m *(t)}\right)^{n}}{\sqrt{n !}}\langle n|
$$


então podemos concluir

$$
e^{m_{\mathbf{i}}^{*}(t) \cdot \tilde{\mathbf{n}}_{1}^{\dagger} \hat{\mathbf{a}}_{1}}\left\langle\alpha_{1}\right|=e^{-\frac{1}{2}\left|\alpha_{1}\right|^{2}} e^{\frac{1}{2}\left|e^{m_{1}(t)} \alpha_{1}\right|^{2}}\left\langle\alpha_{1} e^{m_{1}(t)}\right|
$$

Analogamente

$$
e^{m_{2}^{*}(t) \cdot \bar{a}_{2}^{\dagger} \bar{a}_{2}}\left\langle\alpha_{2}\right|=e^{-\frac{1}{2}\left|\alpha_{2}\right|^{2}} e^{\frac{1}{2}\left|e^{m_{2}(t)} \alpha_{2}\right|^{2}}\left\langle\alpha_{2} e^{m_{2}(t)}\right|
$$

- $3^{a}$ Maneira - Superoperadores "tipo 11"ou "tipo 22" com ponto no meio.

Para mostra que a exponencial desses superoperadores atuando num estado coerente nào altera o estado, faremos o segunte cálculo:

1. Superoperadores $\hat{a}_{1} \cdot \hat{a}_{1}^{\dagger}, \hat{a}_{2} \cdot \hat{a}_{2}^{\dagger}, \hat{a}_{1} \cdot \hat{a}_{2}^{\dagger}$ e $\hat{a}_{2} \cdot \hat{a}_{1}^{\dagger}$

$$
\begin{aligned}
e^{j_{1}(t) \hat{\mathbf{a}}_{1} \cdot \hat{\mathbf{a}}_{1}^{\dagger}}\left|\alpha_{1}\right\rangle\left\langle\alpha_{1}\right| & =\sum_{k} \frac{j_{1}(t)^{k}}{k !}\left(\hat{\mathbf{a}}_{1}\right)^{k}\left|\alpha_{1}\right\rangle\left\langle\alpha_{1}\right|\left(\hat{\mathbf{a}}_{1}^{\dagger}\right)^{k} \\
& =e^{j_{1}(t)\left|\alpha_{2}\right|^{2}}\left|\alpha_{1}\right\rangle\left\langle\alpha_{1}\right| .
\end{aligned}
$$

Analogamente:

$$
\begin{aligned}
e^{j_{2}(t) \hat{\mathbf{a}}_{2} \cdot \hat{\mathbf{a}}_{2}^{\dagger}}\left|\alpha_{2}\right\rangle\left\langle\alpha_{2}\right| & =\sum_{k} \frac{j_{2}(t)^{k}}{k !}\left(\hat{\mathbf{a}}_{2}\right)^{k}\left|\alpha_{2}\right\rangle\left\langle\alpha_{2}\right|\left(\hat{\mathbf{a}}_{2}^{\dagger}\right)^{k} \\
& =e^{j j_{2}(t)\left|\alpha_{2}\right|^{2}}\left|\alpha_{2}\right\rangle\left\langle\alpha_{2}\right|, \\
e^{z(t) \hat{\mathbf{a}}_{2} \cdot \hat{\mathbf{a}}_{1}^{\dagger}}\left|\alpha_{2}\right\rangle\left\langle\alpha_{1}\right| & =\sum_{k} \frac{z(t)^{k}}{k !}\left(\hat{\mathbf{a}}_{2}\right)^{k}\left|\alpha_{2}\right\rangle\left\langle\alpha_{1}\right|\left(\hat{\mathbf{a}}_{1}^{\dagger}\right)^{k} \\
& =e^{z(t) \alpha_{2} \alpha_{1}^{*}}\left|\alpha_{2}\right\rangle\left\langle\alpha_{1}\right| \\
e^{z^{*}(t) \overline{\mathbf{a}}_{1} \cdot \overline{\mathbf{a}}_{2}^{\dagger}}\left|\alpha_{1}\right\rangle\left\langle\alpha_{2}\right| & =\sum_{k} \frac{\left(z^{*}(t)\right)^{k}}{k !}\left(\hat{\mathbf{a}}_{1}\right)^{k}\left|\alpha_{1}\right\rangle\left\langle\alpha_{2}\right|\left(\hat{\mathbf{a}}_{2}^{\dagger}\right)^{k} \\
& =e^{z^{*}(t) \alpha_{2}^{*} \alpha_{1}}\left|\alpha_{1}\right\rangle\left\langle\alpha_{2}\right| .
\end{aligned}
$$

\section{A.3 Evolução de $\rho_{0}=\left|\alpha_{1}\right\rangle\left\langle\alpha_{1}|\otimes| \alpha_{2}\right\rangle\left\langle\alpha_{2}\right|$}

Com o estado inicial em questão, mostraremos a evolução de $\rho(t)$ da equação 4.16.

1. Os superoperadores das equaçōes A.7 e A.9 atuando em $\left|\alpha_{1}\right\rangle\left\langle\alpha_{1}|\otimes| \alpha_{2}\right\rangle\left\langle\alpha_{2}\right|$, resultam:

$$
\begin{aligned}
& e^{q(t) \hat{\mathbf{a}}_{2} \hat{\mathbf{a}}_{1}^{\dagger}} \cdot e^{q^{*}(t) \cdot \hat{\mathbf{a}}_{2}^{\dagger} \hat{\mathbf{a}}_{2}}\left|\alpha_{1}\right\rangle\left\langle\alpha_{1}|\otimes| \alpha_{2}\right\rangle\left\langle\alpha_{2}\right| \\
& \quad=e^{-\left|\alpha_{1}\right|^{2}} e^{\left|\alpha_{1}+q(t) \alpha_{2}\right|^{2}}\left|\alpha_{1}+q(t) \alpha_{2}\right\rangle\left\langle\alpha_{1}+q(t) \alpha_{2}|\otimes| \alpha_{2}\right\rangle\left\langle\alpha_{2}\right|
\end{aligned}
$$


com isto

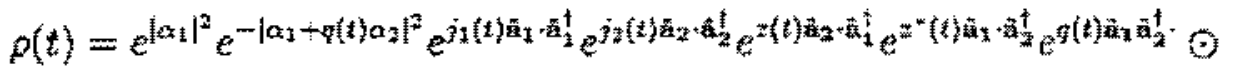

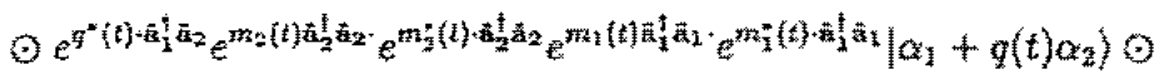

$$
\begin{aligned}
& \text { of }\left|\alpha_{1}+g(t) \alpha_{2}\right| \otimes\left|\alpha_{2}\right|\left\langle\alpha_{2}\right|
\end{aligned}
$$

2. Os superoperadores das equacoes A.11, A.12, A.13 t A.12 atuando em $\left.\left.\mid \alpha_{1}+\eta(t) \alpha_{2}\right) \alpha_{1}+q(t) \alpha_{2}|\otimes| \alpha_{2}\right)\left|\alpha_{2}\right|$. resultam:

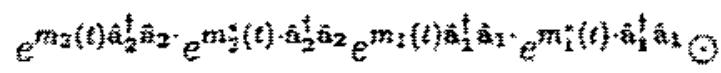

$$
\begin{aligned}
& \left.\odot \mid \alpha_{1}+g(t) \alpha_{2}\right)\left\langle\alpha_{1}+q(t) \alpha_{2}|\otimes| \alpha_{2}\right)\left|\alpha_{2}\right|=
\end{aligned}
$$

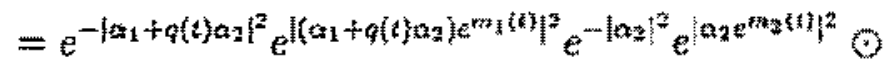

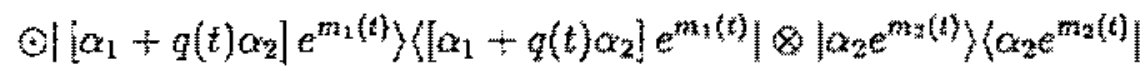

com isto

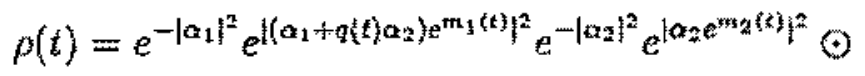

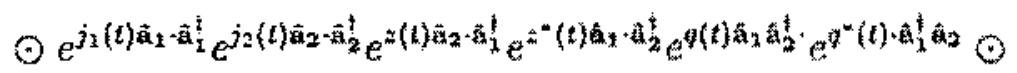

$$
\begin{aligned}
& \odot\left|\left[\alpha_{1}+q(t) \alpha_{2}\right] e^{m_{1}(t)}\right\rangle\left\langle\left[\alpha_{1}+q(t) \alpha_{2}\right] e^{m_{1}(t)}|\otimes| \alpha_{2} e^{m t_{2}(t)}\right\rangle\left\langle\alpha_{2} e^{m_{2}(t)}\right|
\end{aligned}
$$

3. Os superoperadores das equaçöes A.8 e A.10 atuando em $\left|\left[\alpha_{1}+q(t) \alpha_{2}\right\} e^{m_{1}(t)}\right\rangle\left\langle\left[\alpha_{1}+q(t) \alpha_{2}\right\} e^{m_{1}(t)}|\otimes| \alpha_{2} e^{m_{2}(t)}\right\rangle\left\langle\alpha_{2} e^{m_{2}(t)}\right|$, resultam

$$
\begin{aligned}
& \left.e^{q(t) \hat{\alpha}_{2} \hat{a}_{-}^{t}} e^{q^{*}(t) \cdot \hat{\alpha}_{1}^{t} \hat{a}_{2}} \mid\left(\alpha_{1}+q(t) \alpha_{2}\right) e^{m_{1}\{t)}\right)\left\langle\left(\alpha_{1}+q(t) \alpha_{2}\right) e^{m_{1}(t)}\right| \odot
\end{aligned}
$$

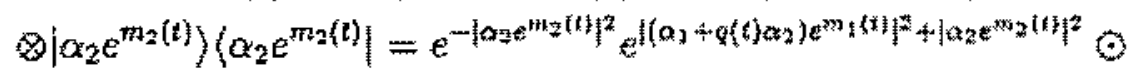

$$
\begin{aligned}
& \text { 이 }\left[\alpha_{1}+q(t) \alpha_{2}\right] e^{m_{1}(i)} /\left[\alpha_{1}+q(t) \alpha_{2}\right] e^{m_{2}(t)} \mid \otimes \\
& \left.\checkmark \mid \alpha_{2} e^{m z_{2}(t)}+q(t)\left[\alpha_{1}+q(t) \alpha_{2}\right] e^{m_{1}(t)}\right)\left(\alpha_{2} e^{m m_{2}(t)}+q(t)\left[\alpha_{1}+q(t) \alpha_{2}\right] e^{m_{1}(t)}\right.
\end{aligned}
$$

Definindo

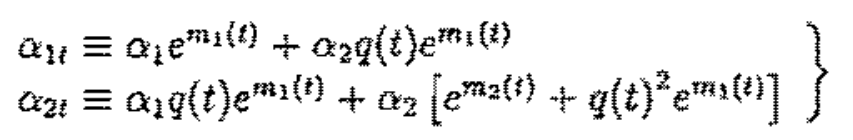

teremos

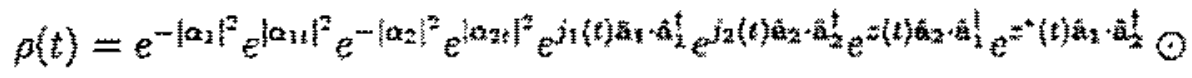

$$
\begin{aligned}
& \left.0 \mid \alpha_{1 t}\right)\left(\alpha_{1 t}|\otimes| \alpha_{2 t}\right)\left(\alpha_{2 t} \mid\right.
\end{aligned}
$$

4. Os superoperadores das equações das equaçöes A.17 e A.18 atuando em $\left|\alpha_{1 t}\right\rangle\left\langle\alpha_{1 t}|\otimes| \alpha_{z t}\right\rangle\left\langle\alpha_{2 l}\right|$, resultarn:

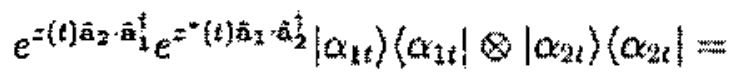

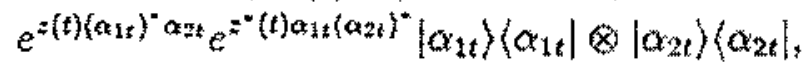


tom isto

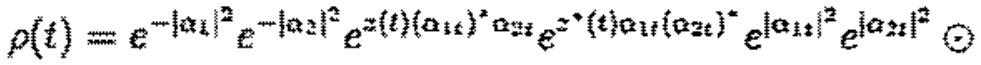

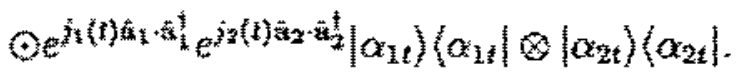

5. Os superoperadores das equaçōes A.15 e A.16 atuando en $\left|\alpha_{1 t}\right\rangle\left\langle\alpha_{1 t}|\otimes| \alpha_{2 t}\right\rangle\left\langle\alpha_{2 t}\right|$, resultam:

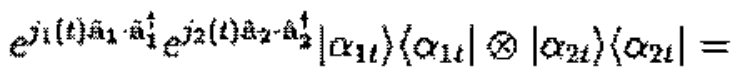

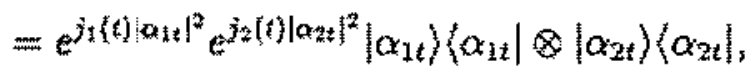

com isto

$$
\begin{aligned}
& p(t)=e^{-\left|\alpha_{1}\right|^{z}} e^{-\left|\alpha_{2}\right|^{2}} e^{z(t)\left(\alpha_{11}\right)^{*} \alpha_{2 t}} e^{z^{*}(t) \alpha_{11}\left(\alpha_{2}\right)^{*}} \odot \\
& \odot e^{\left(j_{1}(t)+1\right)\left|\alpha_{2 i}\right|^{2}} e^{\left(j_{2}(t)+1\right)\left|\alpha_{2 t}\right|^{2}}\left|\alpha_{1 t}\right\rangle\left\langle\alpha_{12}|\otimes| \alpha_{2 t}\right\rangle\left\langle\alpha_{2 t}\right| .
\end{aligned}
$$

Una vez que $\operatorname{Tr} p(t)=1$, entäo podemos determinar os coeficientes $j_{2}(t), j_{1}(t)$, $z(t)$

$$
\left.\begin{array}{l}
j_{2}(t)=\left(1+|q(t)|^{2}\right)\left(\left|e^{m_{2}(t)}\right|^{-2}\right)-1 \\
j_{1}(t)=\left|e^{-m_{1}(t)}+q(t)^{2} e^{-m_{7}(t)}\right|^{2}+\left(|q(t)|^{2}\right)\left(\left|e^{m_{2}(t)}\right|^{-2}\right)-1 \\
z(t)=-q(t) e^{-\left(m_{i}(t)+m_{2}(t)\right)}-q^{*}(t)\left(1+|q(t)|^{2}\right)\left|e^{m_{2}(t)}\right|^{-2}
\end{array}\right\}
$$

Assim, podenos finalmente obter a evoluçà̃o temporal do estado coerente objeto desta seçăo, escrever:

$$
\rho(t)=\left|\alpha_{1 t}\right| \alpha_{1 t}|\otimes| \alpha_{2 t}|| \alpha_{2 t} \mid
$$




\section{Apêndice B}

\section{Aplicação da técnica 3.2.2 para dois osciladores}

\section{B.1 Relação de comutação}

Dadas as relaçoes de comutaçäo entre os superoperadores bosônicos,

$$
\begin{aligned}
& {\left[\left(\hat{a}_{1} \cdot\right),\left(\hat{a}_{1}^{\dagger} \cdot\right)\right]=(1)=1} \\
& {\left[\left(\cdot \hat{a}_{1}\right),\left(\cdot \hat{a}_{1}^{\dagger}\right)\right]=-(\cdot 1)=-1} \\
& {\left[\left(\cdot \hat{a}_{1}\right),\left(\hat{a}_{1}^{\dagger}\right)\right]=\left[\left(\hat{a}_{1} \cdot\right),\left(\cdot \hat{a}_{1}^{\dagger}\right)\right]=0}
\end{aligned}
$$

construiremos, a seguir, uma tabela de conutaça entre os superoperadores,

$$
\begin{aligned}
& a_{1}^{\dagger} \hat{a}_{1} \cdot \equiv \tilde{M}_{1}, \quad \cdot \hat{a}_{1}^{\dagger} \hat{a}_{1} \equiv \hat{\mathbb{P}}_{1}, \quad \hat{\mathbf{a}}_{1} \cdot \hat{\mathbf{a}}_{1}^{\dagger} \equiv \hat{\mathbb{J}}_{1}, \quad \hat{\mathbf{a}}_{1} \hat{a}_{2}^{\dagger} \equiv \hat{\mathbb{N}}, \quad \hat{\mathbf{a}}_{1}^{\dagger} \hat{\mathbf{a}}_{2} \cdot \equiv \hat{\mathbf{Q}}, \quad \hat{\mathbf{a}}_{2} \cdot \hat{\mathbf{a}}_{1}^{\dagger} \equiv \hat{\mathbb{Z}}, \\
& \hat{\mathrm{a}}_{2}^{\dagger} \hat{\mathrm{a}}_{2} \cdot \equiv \hat{\mathbb{M}}_{2}, \quad \cdot \hat{\mathrm{a}}_{2}^{\dagger} \hat{\mathrm{a}}_{2} \equiv \hat{\mathbb{P}}_{2}, \quad \hat{\mathrm{a}}_{2} \cdot \hat{\mathrm{a}}_{2}^{\dagger} \equiv \hat{\mathbb{J}}_{2}, \quad \cdot \hat{\mathrm{a}}_{1}^{\dagger} \hat{\mathrm{a}}_{2} \equiv \tilde{\mathbb{N}}_{l}, \quad \cdot \hat{\mathbf{a}}_{1} \hat{\mathrm{a}}_{2}^{\dagger} \equiv \hat{\mathbb{Q}}_{1}, \quad \hat{\mathrm{a}}_{1} \cdot \hat{\mathrm{a}}_{2}^{\dagger} \equiv \hat{\mathbb{Z}}_{l},
\end{aligned}
$$

de interesse ao nosso trabalho.

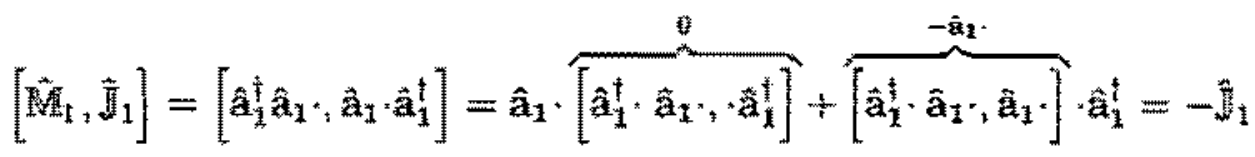

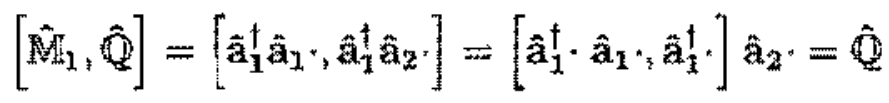

$$
\begin{aligned}
& {\left[\hat{M}_{1}, \hat{N}\right]=\left[\hat{a}_{1}^{\dagger} \hat{a}_{1}, \hat{a}_{1} \hat{a}_{2}^{t}\right]=\left[\hat{a}_{1}^{\dagger}, \hat{a}_{1}, \hat{a}_{1} \cdot\right] \hat{a}_{2}^{\dagger}=-\hat{\mathbb{N}}}
\end{aligned}
$$




$$
\begin{aligned}
& {\left[\tilde{M}_{1}, \hat{z}_{1}\right]=\left[\hat{a}_{1}^{t} \hat{a}_{1}, \hat{a}_{1}, \hat{a}_{2}^{\dagger}\right]=\left[\hat{a}_{1}^{\dagger}, \hat{a}_{1}, \hat{a}_{1^{j}}\right] \cdot \hat{a}_{2}^{\dagger}=-\hat{Z}_{t}}
\end{aligned}
$$

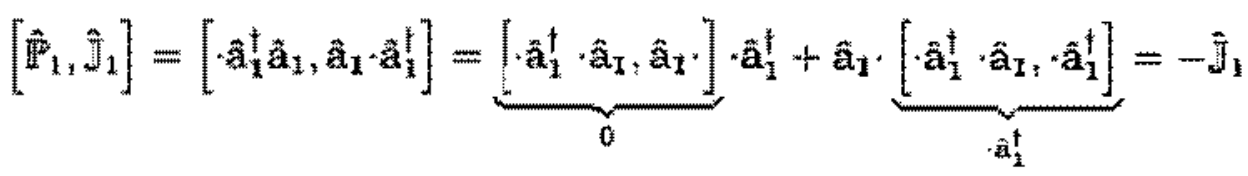

$$
\begin{aligned}
& {\left[\hat{P}_{1}, \hat{Q}_{t}\right]=\left[\cdot \hat{a}_{1}^{\dagger} \hat{a}_{1}, \cdot \hat{a}_{1} \hat{a}_{2}^{t}\right]=\left[\cdot \tilde{a}_{1}^{\dagger} \cdot \hat{a}_{1}, \cdot \hat{a}_{i}\right] \cdot \hat{a}_{z}^{\dagger}=\hat{Q}_{i}}
\end{aligned}
$$

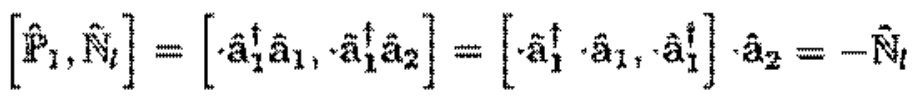

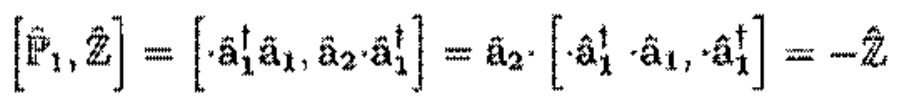

$$
\begin{aligned}
& {\left[\hat{\mathbb{J}}_{1}, \hat{\mathbb{Q}}\right]=\left[\hat{\mathbf{a}}_{1} \cdot \hat{\mathbf{a}}_{1}^{\dagger}, \hat{\mathbf{a}}_{1}^{\dagger} \hat{\mathbf{a}}_{2} \cdot\right]=\left[\hat{\mathbf{a}}_{1} \cdot \cdot \hat{\mathbf{a}}_{1}^{\dagger}, \hat{\mathbf{a}}_{1}^{\dagger}\right] \hat{\mathbf{a}}_{2} \cdot=\ddot{\mathbb{Z}}} \\
& {\left[\hat{J}_{1}, \hat{Q}_{1}\right]=\left[\hat{a}_{2} \cdot \hat{\mathbf{a}}_{1}^{\dagger}, \cdot \hat{\mathbf{a}}_{2} \hat{a}_{2}^{\dagger}\right]=\left[\hat{\mathbf{a}}_{1} \cdot \hat{\mathbf{a}}_{1}^{\dagger}, \cdot \hat{\mathbf{a}}_{1}\right] \cdot \hat{\mathbf{a}}_{2}^{\dagger}=\hat{\mathbb{Z}}_{l}}
\end{aligned}
$$

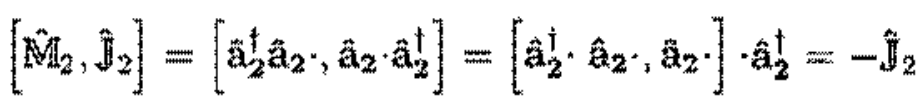

$$
\begin{aligned}
& {\left[\hat{M}_{2}, \mathbb{Q}\right]=\left[\hat{a}_{2}^{\dagger} \hat{a}_{2}, \hat{\mathbf{a}}_{1}^{\dagger} \hat{a}_{2} \cdot\right]=\hat{a}_{1}^{\dagger} \cdot\left[\hat{a}_{2}^{j} \cdot \hat{a}_{2} \cdot, \hat{a}_{2} \cdot\right]=-\hat{\mathbb{Q}}} \\
& {\left[\hat{M}_{L_{2}}, \hat{N}\right]=\left[\hat{a}_{2}^{\dagger} \hat{a}_{2^{*}}, \hat{a}_{1} \hat{a}_{2}^{\dagger}\right]=\hat{a}_{1} \cdot\left[\hat{a}_{2}^{t} \cdot \hat{a}_{2^{*}}, \hat{a}_{2}^{\dagger}\right]=\hat{R}}
\end{aligned}
$$

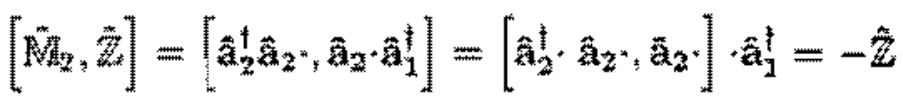

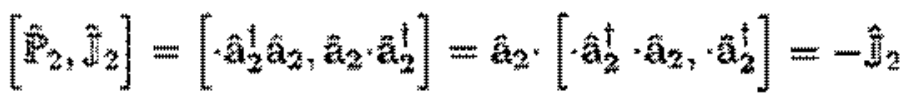




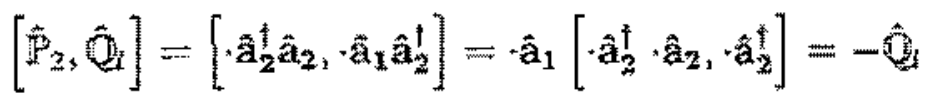

$$
\begin{aligned}
& {\left[\hat{\mathbb{P}}_{2}, \hat{\mathbb{N}}_{1}\right]=\left[-\hat{a}_{2}^{\dagger} \hat{a}_{2}, \hat{a}_{1}^{\dagger} \hat{a}_{2}\right]=\hat{a}_{1}^{\dagger}\left[-\hat{a}_{2}^{\dagger} \cdot \hat{a}_{2}, \hat{a}_{2}\right]=\hat{N}_{1}}
\end{aligned}
$$

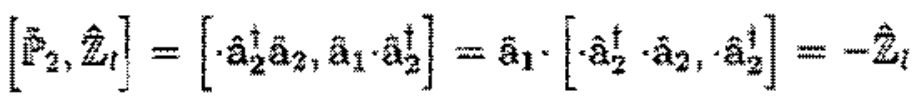

$$
\begin{aligned}
& {\left[\hat{\mathbb{R}}_{2}, \hat{\mathbb{N}}\right]=\left[\hat{\mathrm{a}}_{2} \cdot \hat{\mathbf{a}}_{2}^{\dagger}, \hat{\mathrm{a}}_{x} \mathrm{a}_{2}^{\dagger}\right]=\hat{\mathbf{a}}_{1} \cdot\left[\hat{\mathbf{a}}_{2} \cdot \hat{\mathbf{a}}_{2}^{\dagger}, \hat{\mathbf{a}}_{2}^{\dagger}\right]=\hat{\mathbb{Z}}_{i}}
\end{aligned}
$$

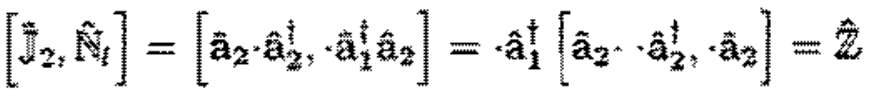

$$
\begin{aligned}
& {[\tilde{N}, \hat{\mathbb{Z}}]=\left[\hat{a}_{1} \hat{a}_{2}^{\dagger}, \hat{a}_{2} \cdot \hat{a}_{1}^{\dagger}\right]=\hat{a}_{2} \cdot\left[\hat{a}_{1} \cdot \hat{a}_{2}^{\dagger} \cdot, \cdot \hat{a}_{1}^{\dagger}\right]+\left[\hat{a}_{1} \cdot \hat{a}_{2}^{\tilde{i}}, \hat{a}_{2} \cdot\right] \cdot \hat{\mathbf{a}}_{1}^{\dagger}=-\hat{J}_{1}}
\end{aligned}
$$

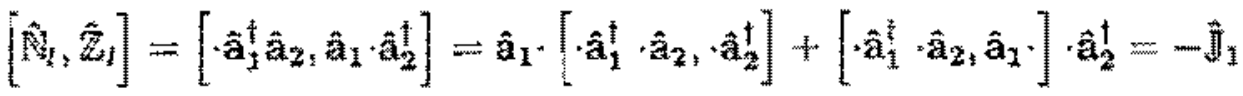

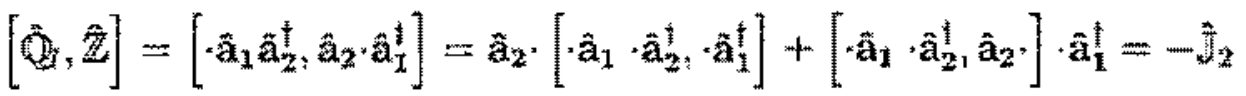

$$
\begin{aligned}
& {\left[\hat{\mathbb{Q}}_{1}, \hat{\mathbb{Z}}_{l}\right]=\left[\hat{\mathrm{a}}_{1}^{\dagger} \hat{\mathrm{a}}_{2} \cdot, \hat{\mathrm{a}}_{1} \cdot \hat{\mathrm{a}}_{2}^{\dagger}\right]=\hat{\mathrm{a}}_{1} \cdot\left[\hat{\mathrm{a}}_{1}^{\dagger} \cdot \hat{\mathrm{a}}_{2} \cdot, \hat{\mathrm{a}}_{2}^{\dagger}\right]+\left[\hat{\mathrm{a}}_{1}^{\dagger} \cdot \hat{\mathrm{a}}_{2} \cdot, \hat{\mathrm{a}}_{1}\right] \cdot \hat{\mathrm{a}}_{2}^{\dagger}=-\hat{\mathfrak{J}}_{2}} \\
& {\left[\hat{\mathbb{N}}_{1}, \hat{Q}\right]=\left[\hat{a}_{1} \hat{a}_{2}^{\dagger}, \hat{a}_{1}^{\dagger} \hat{a}_{2} \cdot\right]=\left[\hat{a}_{1} \cdot \hat{a}_{2}^{\dagger}, \hat{a}_{1}^{\dagger} \cdot\right] \hat{a}_{2}+\hat{a}_{1}^{\dagger} \cdot\left[\hat{a}_{1} \cdot \hat{a}_{2}^{\dagger}, \hat{a}_{2}\right]=\hat{M}_{2}-\hat{M}_{1}}
\end{aligned}
$$

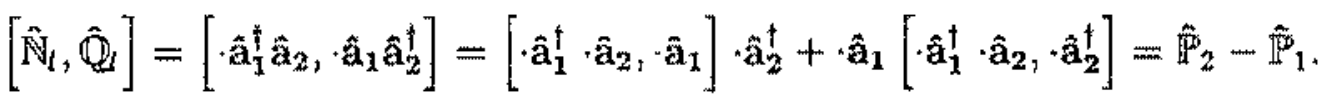

79 
Sendo as demais relaçoes de comutacão utilizadas no nosso trabalho iguais a zero, podemos resumir todas as relaçoes de comutaçâto calculadas na tabela que segue:

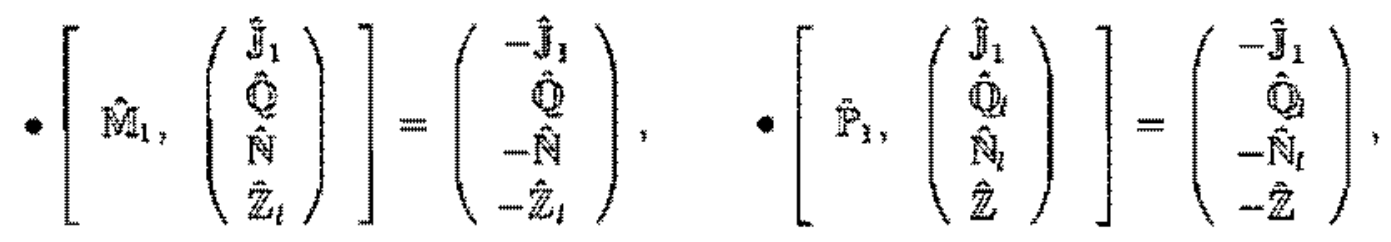

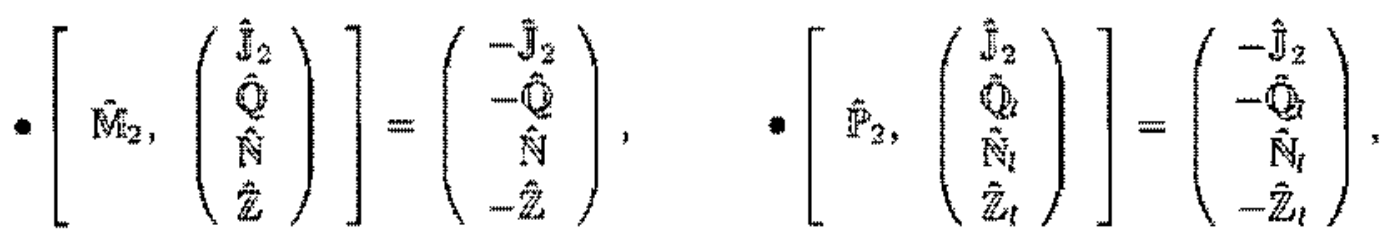

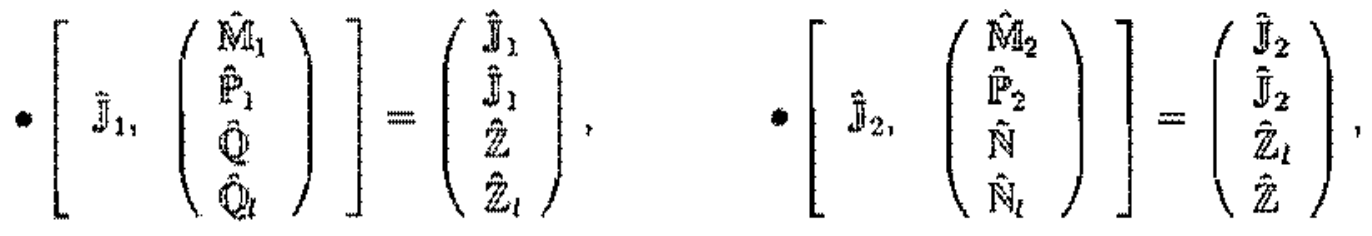

$$
\begin{aligned}
& \cdot\left[\hat{\mathbb{Z}}_{1}\left(\begin{array}{l}
\hat{\mathbb{P}}_{1} \\
\hat{\mathbb{M}}_{2} \\
\hat{\mathbb{N}} \\
\hat{\mathbb{Q}}_{1}
\end{array}\right)\right]=\left(\begin{array}{l}
\hat{\mathbb{Z}} \\
\hat{\mathbb{Z}} \\
\hat{\mathbb{H}}_{1} \\
\hat{\mathbb{Z}}_{2}
\end{array}\right), \quad \cdot\left[\hat{\mathbb{Z}}_{l},\left(\begin{array}{c}
\tilde{\mathbb{M}}_{2} \\
\hat{\mathbb{P}}_{2} \\
\hat{\mathbb{N}}_{l} \\
\hat{\mathbb{Q}}
\end{array}\right)\right]=\left(\begin{array}{l}
\hat{\mathbb{Z}}_{l} \\
\hat{\mathbb{Z}}_{l} \\
\hat{\mathbb{J}}_{1} \\
\hat{\mathbb{Z}}_{2}
\end{array}\right),
\end{aligned}
$$

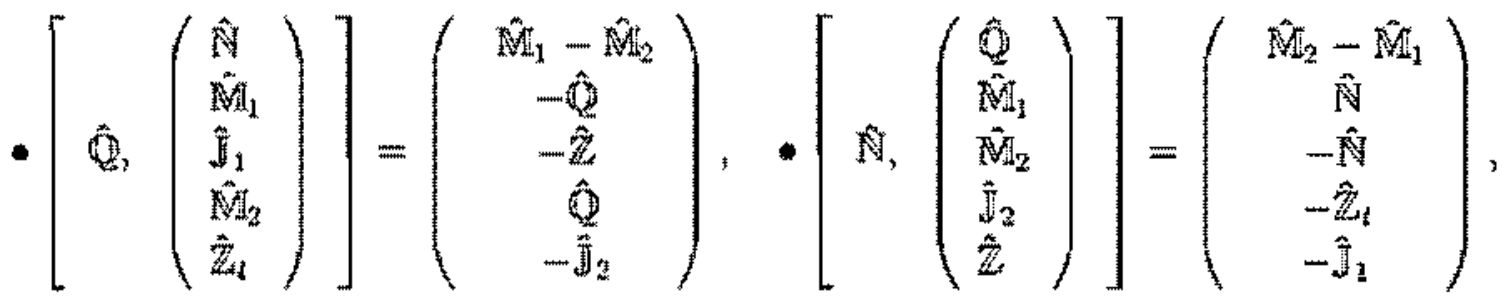

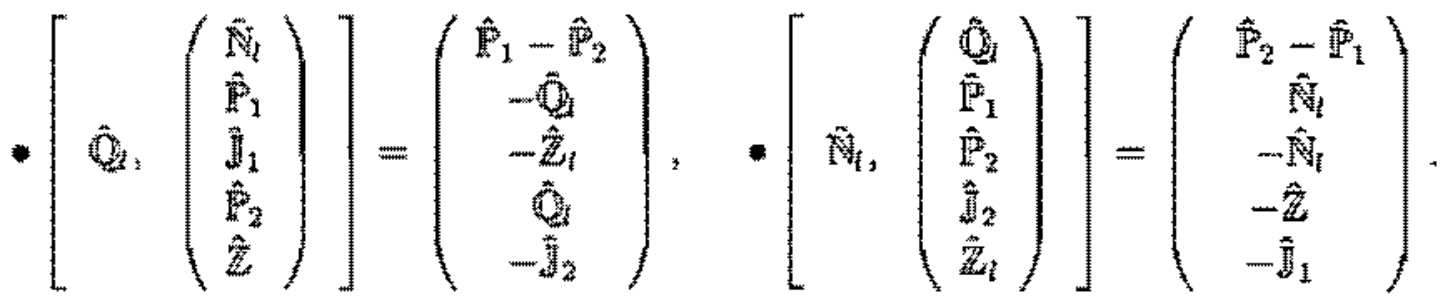




\section{B.2 Desacoplamento de dois osciladores}

O liuvilliano encontrado na deduçẵo da equaçäo Mestra 4.15, pode ser ordenado da seguinte maneira:

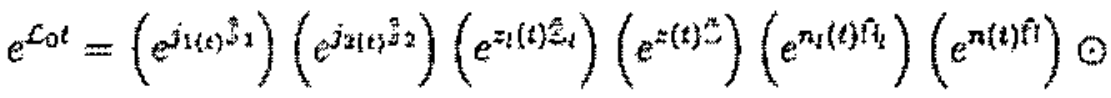

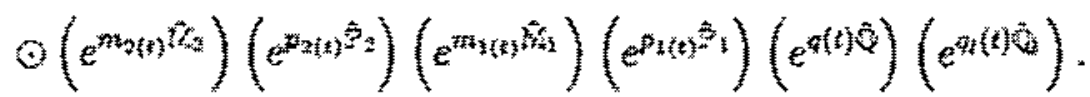

Para calcularmos os coeficientes do ordenamento dado, utilizaremos a técnica de derivaçăo de parâmetros apresentada no capitulo 3 .

O objetivo desta técnica é obter através de derivaçăo, a messma exponencial em ambos os lados da igualdade e manter os superoperadores que não estiverem na cabeça das exponenciais, por conta da derivada, sempre a esquerda das exponenciais, para que possam ficar "livres", serem comparados e seus coeficientes determinados. Para atingir este objetivo de uma maneira prática definiremos:

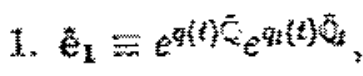

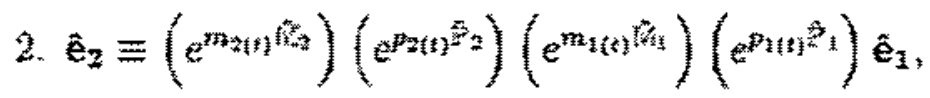
3. $\hat{\mathrm{e}}_{\mathrm{s}} \equiv e^{\mathrm{n}(\mathrm{t}) \mathrm{n}} \hat{\mathrm{e}}_{2,}$
4. $\hat{\mathrm{e}}_{4} \equiv e^{\mathrm{n}_{2}(t) \hat{H}_{4}} \hat{\mathrm{e}}_{3}$
5. $\hat{\mathrm{e}}_{\mathfrak{5}} \equiv e^{z(t) \hat{\hat{E}}} \hat{\mathrm{e}}_{4}$

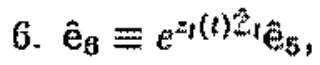

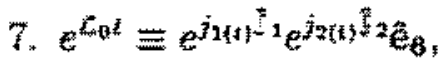

en seguida faremos a derivada no tempo de cada item definido, por exemplo $\dot{\hat{\mathrm{e}}}_{1}$, escrevendo o resultado como um produto de um superoperador vezes o proprio item definido, $\hat{e}_{1}$. Procuraremos explicar detalhadamente ate a derivada da segunda definiçăo e depois utilizaremos o mesmo raciocinio para as derivadas das definiçōes seguintes até que consigamos escrever $\mathcal{L}_{0} e^{\mathcal{L}_{0} t}=\left(\sum\right.$ de superoperadores $) e^{\mathcal{L}_{0} t}$ e determinarmos os valores dos coenicientes.

1. A derivada no tempo para $\hat{e}_{1}$ é dada por.

$$
\left(\frac{d}{d t} \hat{e}_{1}\right)=\left[\left(\frac{d}{d t} q(t)\right) \hat{Q}+\left(\frac{d}{d t} q_{t}(t)\right) \hat{Q}_{t}\right] \hat{\mathrm{e}}_{1} \text {. }
$$

atingindo o objetivo de maneira direta, pois de acordo com as equaçôs B.I, a relaçāo de comutação entre estes superoperadores é zero.

2. A derivada no tempo para $\hat{e}_{2}$ é dada por:

$$
\begin{aligned}
& \left(\frac{d}{d t} \hat{\mathbf{e}}_{2}\right)=\left[\left(\frac{d}{d t} m_{1}(t)\right) \hat{\mathbf{M}}_{1}+\left(\frac{d}{d t} m_{2}(t)\right) \hat{M}_{2}+\left(\frac{d}{d t} p_{1}(t)\right) \hat{\mathbb{P}}_{1}+\left(\frac{d}{d t} p_{2}(t)\right) \hat{\mathbb{E}}_{2}\right] \hat{\mathbf{e}}_{2}
\end{aligned}
$$

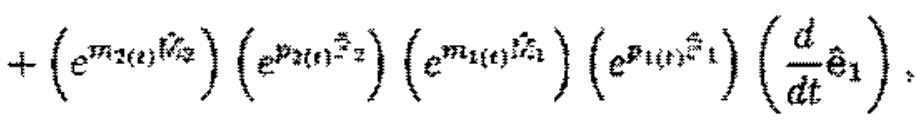


observamos que o primeiro tarmo atinge o objetivo proposto, enquanto que no segundo termo podemos substiturir $\hat{\mathrm{e}}_{1}$, calculado no item 1 .

$$
\begin{aligned}
& \left(\frac{d}{d t} \hat{\mathbf{e}}_{2}\right)=\left[\left(\frac{d}{d t} m_{1}(t)\right) \hat{\mathbf{M}}_{1}+\left(\frac{d}{d t} m_{2}(t)\right) \hat{\mathbf{M}}_{2}+\left(\frac{d}{d t} p_{1}(t)\right) \hat{\mathbb{P}}_{1}+\left(\frac{d}{d t} p_{2}(t)\right) \hat{R}_{2}\right] \hat{\mathbf{e}}_{2}
\end{aligned}
$$

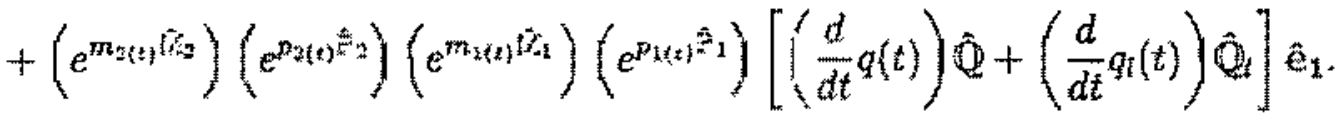

Para podermos escrever o segundo termo en funça de $\hat{e}_{2}$, temos que trans portar as exponenciais para a direita dos superoperadores $\hat{Q}$ e $\hat{Q}$. $\hat{O}$ artificio usado é mostrado a seguir.

$$
\begin{aligned}
& \left(\frac{d}{d t} \hat{e}_{2}\right)=\left[\left(\frac{d}{d t} m_{1}(t)\right) \vec{M}_{1}+\left(\frac{d}{d t} m_{2}(t)\right) \hat{M}_{2}+\left(\frac{d}{d t} p_{1}(t)\right) \hat{P}_{1}+\left(\frac{d}{d t} p_{2}(t)\right) \hat{P}_{2}\right] \hat{e}_{2}
\end{aligned}
$$

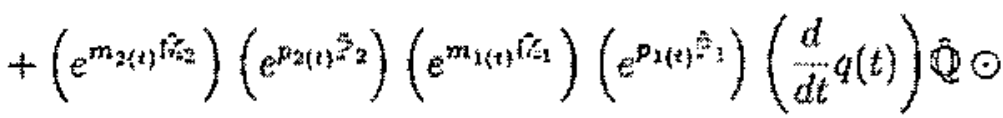

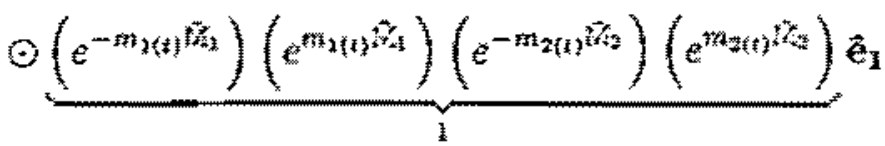

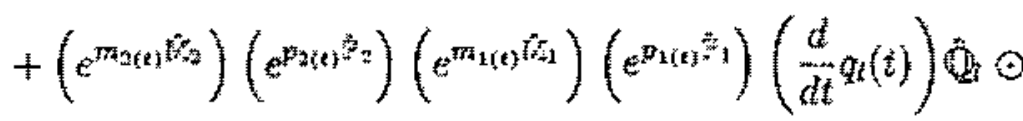

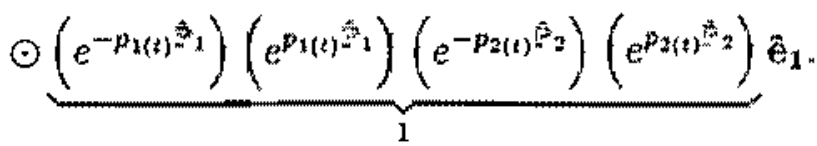

As relaçoses de comutação en B.1, nos indica que os superoperadores P. $_{1}$, $\hat{M_{1}}, \hat{A l}_{2}$ comutan entre sí com os superoperadores $\hat{Q}$ a $\hat{Q}$ respectivamente, entato:

$$
\begin{aligned}
& \left(\frac{d}{d t} \hat{\mathrm{e}}_{2}\right)=\left[\left(\frac{d}{d t} m_{1}(t)\right) \hat{M}_{1}+\left(\frac{d}{d t} m_{2}(t)\right) \hat{M}_{2}+\left(\frac{d}{d t} m_{1}(t)\right) \hat{p}_{t}+\left(\frac{d}{d t} p_{2}(t)\right) \hat{P}_{2}\right] \hat{\epsilon}_{2}
\end{aligned}
$$

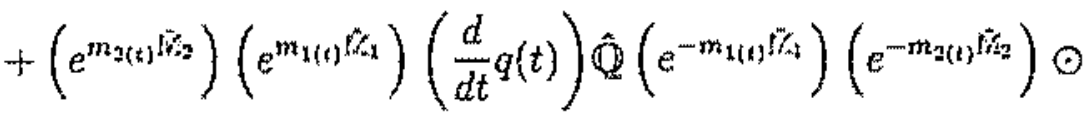

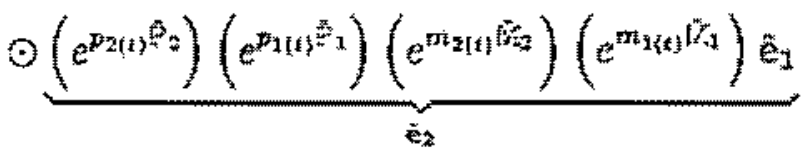

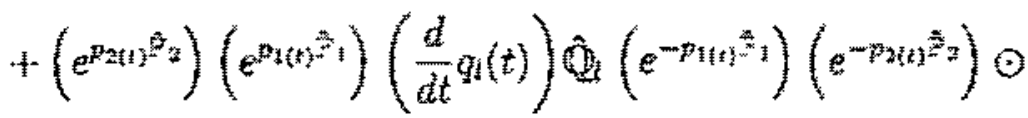

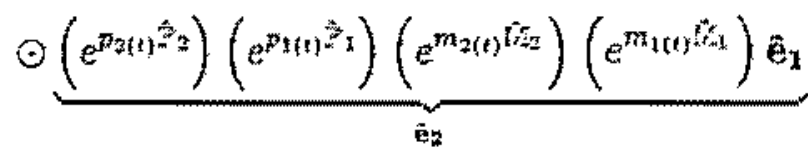

82 


$$
\begin{aligned}
& \left(\frac{d}{d t} \hat{\mathrm{e}}_{2}\right)=\left[\left(\frac{d}{d t} m_{1}(t)\right) \hat{\mathbf{M}}_{1}+\left(\frac{d}{d t} m_{2}(t)\right) \hat{\mathbf{M}}_{2}+\left(\frac{d}{d t} p_{1}(t)\right) \hat{M}_{1}+\left(\frac{d}{d t} p_{2}(t)\right) \hat{\mathbf{P}}_{2}\right] \hat{\mathrm{e}}_{2}
\end{aligned}
$$

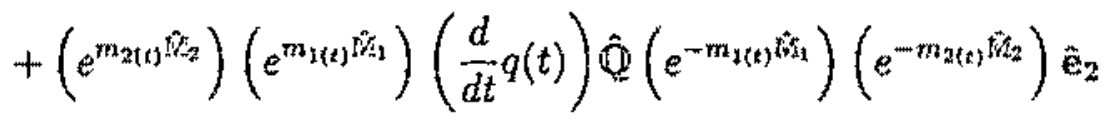

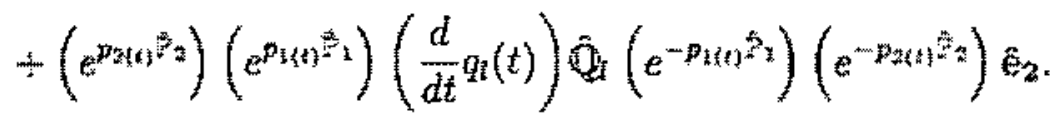

Para que os superoperadores, 0 e 0 , fquem a esquerda das exponenciais, utllizaremos a transformaçäo de semelhança 3.5 o as relaçoes de comutaçăo dadas am B.1,como segue

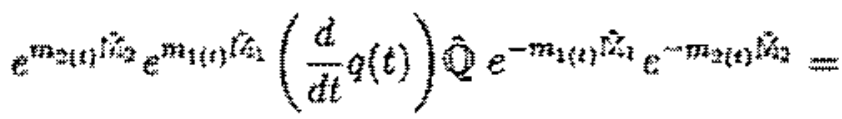

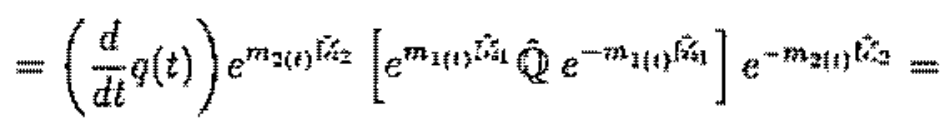

$$
\begin{aligned}
& =\left(\frac{d}{d t} q(t)\right) e^{m_{2(1)} t^{*}}[\underbrace{\left(1+\frac{m_{1}(t)}{1 !}+\frac{m_{1}(t)^{2}}{2 !}+\ldots\right)}_{=\epsilon^{m_{2}(t)}}] e^{-m_{2(t)} m_{2}} \\
& =\left(\frac{d}{d t} q(t)\right) e^{m(t)}\left[e^{m_{2 i t} \hat{m}_{q}} \hat{Q} e^{-m_{3(t)} \hat{m}_{z}}\right] \\
& =\left(\frac{d}{d t} p(t)\right) e^{m_{3}(n)}\left[\left(1-\frac{m_{2}(b)}{1 !}+\frac{m_{2}(t)^{2}}{2 !}-\ldots\right) \hat{Q}\right] \\
& =\left(\frac{d}{d t} q(t)\right) e^{m_{1}(t)-m_{3}(t)} \hat{Q}
\end{aligned}
$$

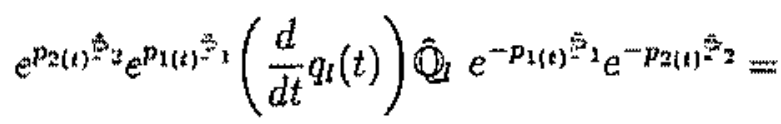

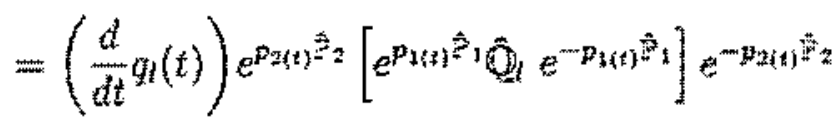

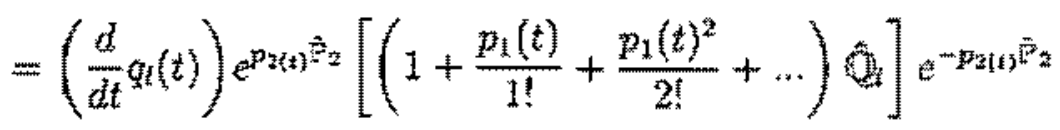

83 


$$
\begin{aligned}
& =\left(\frac{d}{d t} a t(t)\right) e^{p_{1}(t)}\left[e^{p_{2(t)} z_{2}} \hat{Q}_{t} e^{-p_{2}(t)^{p_{2}}}\right] \\
& =\left(\frac{d}{d t} q_{t}(t)\right) e^{p_{1}(n)}\left[\left(1-\frac{p_{3}(t)}{1 !}+\frac{p_{2}(t)^{2}}{2 !}-\ldots\right) \omega_{4}\right] \\
& =\left(\frac{d}{d t} d t(t)\right) e^{p_{1}(t)-p a(t)}(t)
\end{aligned}
$$

e finalmente atinjir nosto objetivo escrevendo:

$$
\begin{aligned}
& \left(\frac{d}{d t} \tilde{e}_{2}\right)=\left[\left(\frac{d}{d t} m_{1}(\hat{t})\right) \hat{M}_{1}+\left(\frac{d}{d t} m_{2}(t)\right) \hat{M}_{2}+\left(\frac{d}{d t} m_{1}(t)\right) \hat{\mathbb{P}}_{1}+\left(\frac{d}{d z} p_{2}(t)\right) \hat{\mathbb{P}}_{2}\right] \hat{e}_{2} \\
& +\left[\left(\frac{d}{d t} q(t)\right) e^{\left(m_{1}(t)-m_{2}(t)\right)} \mathrm{Q}+\left(\frac{d}{d t} q(t)\right) e^{\left(p_{1}(t)-m p_{2}(t)\right)} \mathrm{Q}_{4}\right] \hat{\mathbf{e}}_{2}
\end{aligned}
$$

Para as clerivadas das definiçöes restantes, utilizaremos o mesmo roteiro que foi detalhado para $e_{2}$.

3. A derivada no tempo da terceira definição $\left.\hat{e}_{3} \equiv e^{n ! t}\right)^{h} \hat{e}_{2}$ é dada por:

$$
\begin{aligned}
& \left(\frac{d}{d t} \hat{\mathrm{e}}_{s}\right)=\left(\frac{d}{d t} n(t)\right) \hat{N}_{\hat{\mathrm{e}}} \hat{\mathrm{e}}_{3}+\left(e^{n(t) \hat{n}}\right)\left(\frac{d}{d t} \hat{\mathrm{e}}_{2}\right) \\
& =\left(e^{\mathrm{n}(t) \hat{n}}\right)\left[\left(\frac{d}{d t} m_{1}(t)\right) \hat{M}_{1}+\left(\frac{d}{d t} m m_{2}(t)\right) \hat{M}_{2}+\left(\frac{d}{d t} q(t)\right) e^{\left(m_{1}(t)-m_{2}(t)\right.}(\hat{Q}] \hat{e}_{2}\right. \\
& +\left(e^{n(t)}\right)\left[\left(\frac{d}{d t} p_{2}(t)\right) \hat{\mathbb{E}}_{2}+\left(\frac{d}{d t} p_{1}(t)\right) \hat{p}_{1}+\left(\frac{d}{d t} q_{t}(t)\right) e^{\left(p_{1}(t)-p_{2}(t)\right)} \hat{\mathrm{D}}\right] \tilde{\mathrm{e}}_{2} \\
& +\left(\frac{d}{d t} n(t)\right) \hat{N} \vec{e}_{3}
\end{aligned}
$$

Utilizando os artificios feitos para o segundo caso e as relaçoes de comutaçầ dadas em B.1, teremos:

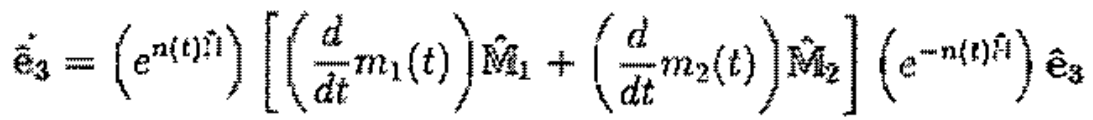

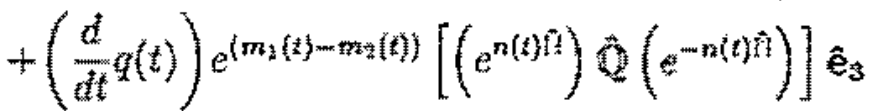

$$
\begin{aligned}
& +\left[\left(\frac{d}{d t} p_{2}(t)\right) \hat{P}_{2}+\left(\frac{d}{d t} p_{1}(t)\right) p_{1}+\left(\frac{d}{d t} q_{1}(t)\right) e^{\left(p_{1}(t) \sim p_{2}(t)\right)} Q_{1}\right] \text { tas }
\end{aligned}
$$




$$
+\left(\frac{d}{d t} n(t)\right) \hat{\mathbb{N}} \hat{e}_{3}
$$

Da transformação de semelhança 3.3 podemos transportar os superoperadores $\widehat{M}_{1}, \hat{M}_{2}, 0$ para e esquerda das exponenciais, como nostramos a seguir,

$$
\begin{aligned}
& \left(e^{n(t) n}\right)\left(\frac{d}{d t} m_{1}(t)\right) \hat{M}_{1}\left(e^{-n(t) n}\right)=\left(\frac{d}{d t} m_{1}(t)\right) \hat{M}_{1}+\left(\frac{d}{d t} m_{1}(t)\right) n(t) \hat{\mathbb{N}} \\
& \left(e^{n(t) n}\right)\left(\frac{d}{d t} m_{2}(t)\right) \hat{M}_{2}\left(e^{-n(t) \hat{H}}\right)=\left(\frac{d}{d t} m_{2}(t)\right) \hat{M}_{2}-\left(\frac{d}{d t} m_{2}(t)\right) n(t) \hat{N} \\
& \left(e^{n(t) n}\right)\left(\frac{d}{d t} q(t)\right) e^{\left(p_{1}(t)-p_{2}(t)\right)} \hat{Q}\left(e^{-n(t) n}\right)= \\
& =\left(\frac{d}{d t} q(t)\right) e^{\left(p_{1}(t)-p_{2}(t)\right)}\left(\hat{Q}+n(t) \hat{M}_{2}-n(t) \hat{M}_{1}-n(t)^{2} \hat{\mathbb{N}}\right)
\end{aligned}
$$

e atijir nosso objetivo escrevendo:

$$
\begin{aligned}
& \left(\frac{d}{d t} \hat{\mathbf{e}}_{3}\right)=\tilde{\mathbb{M}}_{1}\left[\left(\frac{d}{d t} m_{1}(t)\right)-n(t)\left(\frac{d}{d t} q(t)\right) e^{\left(m_{1}(t)-m_{1}(t)\right\}}\right] \hat{\mathbf{e}}_{3} \\
& +\hat{\mathrm{M}}_{2}\left[\left(\frac{d}{d t} m_{2}(t)\right)+n(t)\left(\frac{d}{d t} q(t)\right) e^{\left(m_{1}(t)-m_{2}(t)\right)}\right] \hat{\mathrm{e}}_{3} \\
& +Q\left[\left(\frac{d}{d t} q(t)\right) e^{\left(m_{2}(t)-m_{2}(t)\right)}\right] \hat{\mathrm{e}}_{3} \\
& +\hat{\mathbb{N}}\left[\left(\frac{d}{d t} n(t)\right)-n(t)^{2}\left(\frac{d}{d t} q(t)\right) e^{\left(m_{1}(t)-m_{2}(t)\right)}\right] \tilde{\mathrm{e}}_{\mathfrak{3}} \\
& +N\left[\left(\frac{d}{d t} m_{1}(t)\right) n(t)-\left(\frac{d}{d t} m_{2}(t)\right) n(t)\right] \hat{e}_{3} \\
& +\left[\left(\frac{d}{d t} p_{2}(t)\right) \hat{\mathbb{P}}_{2}+\left(\frac{d}{d t} p_{1}(t)\right) \hat{\mathbb{P}}_{1}+\left(\frac{d}{d t} p(t)\right) e^{\left(p_{1}(t)-p_{2}(t)\right)}\right] \hat{\mathbf{e}}_{3}
\end{aligned}
$$

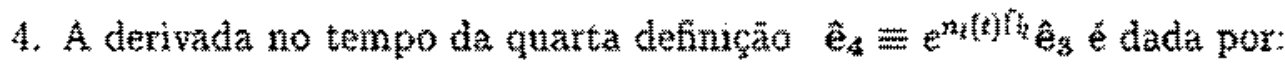

$$
\begin{aligned}
& \left(\frac{d}{d t} \hat{\mathrm{e}}_{4}\right)=\left(e^{r_{t} t m m_{t}}\right)\left(\frac{d}{d t} \hat{\mathrm{e}}_{3}\right)+\left(\frac{d}{d t} n_{t}(t)\right) \hat{N}_{t} \hat{\mathrm{e}}_{4} \\
& =\left(e^{n_{1}(t)}\right) \hat{\mathbb{M}}_{1}\left[\left(\frac{d}{d t} m_{1}(t)\right)-n(t)\left(\frac{d}{d t} t(t)\right) e^{\left(m_{1}(t)-m_{2}(t)\right.}\right] \overrightarrow{\mathrm{e}}_{3}
\end{aligned}
$$




$$
\begin{aligned}
& +\left(e^{n_{1}(t) t_{t}}\right) \tilde{M}_{2}\left[\left(\frac{d}{d t} m_{2}(t)\right)+n(t)\left(\frac{d}{d t} q(t)\right) e^{\left(m_{1}(t)-m_{2}(t)\right]}\right] \tilde{c}_{3} \\
& +\left(e^{n_{i}(t) \eta_{i}}\right) \hat{Q}\left[\left(\frac{d}{d t} q(t)\right) e^{\left(m_{1}(t) \cdots m_{2}(t)\right)}\right] \hat{\omega}_{3}
\end{aligned}
$$

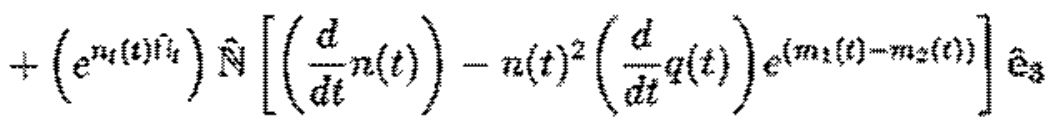

$$
\begin{aligned}
& +\left(e^{n_{1}(t) \bar{m}_{t}}\right) \hat{\mathbb{N}}\left[n(t)\left(\frac{d}{d t} m_{1}(t)\right)-n(t)\left(\frac{d}{d t} m_{2}(t)\right)\right] \mathrm{e}_{3}
\end{aligned}
$$

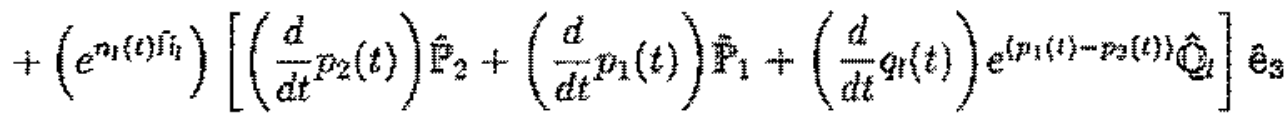

$$
\begin{aligned}
& +\left(\frac{d}{d t} n_{1}(t)\right) \hat{N}_{i} \hat{\mathrm{e}}_{4}
\end{aligned}
$$

Utilizando os artifícios já mostrados e as relaçöez de comutaçäo dadas en B. $I$, teremos:

$$
\begin{aligned}
& \left(\frac{d}{d t} \hat{\mathbf{e}}_{4}\right)=\hat{\mathbb{M}}_{1}\left[\left(\frac{d}{d t} m_{1}(t)\right)-n(t)\left(\frac{d}{d t} q(t)\right) e^{\left(m_{1}(t)-m_{2}(t)\right)}\right] \hat{\mathbf{e}}_{4} \\
& +\hat{\mathrm{M}}_{2}\left[\left(\frac{d}{d t} m_{2}(t)\right)+n(t)\left(\frac{d}{d t} q(t)\right) e^{\left(m_{1}(t)-m_{2}(t)\right)}\right] \hat{\mathbf{e}}_{4} \\
& +0\left[\left(\frac{d}{d t} q(t)\right) e^{\left(m_{1}(t)-m_{3}(t)\right\}}\right] \hat{e}_{A} \\
& +\hat{N}\left[\left(\frac{d}{d t} n(t)\right)-n(t)^{2}\left(\frac{d}{d t} q(t)\right) e^{\left(m_{1}(t)-m_{2}(t)\right)}\right] \hat{a}_{4} \\
& +\mathbb{N}\left[n(t)\left(\frac{d}{d t} m_{1}(t)\right)-n(t)\left(\frac{d}{d t} m_{2}(t)\right)\right] \hat{e}_{4} \\
& +\left(e^{n_{3}(t) \hat{n}_{4}}\right)\left[\left(\frac{d}{d t} p_{2}(t)\right) \hat{\mathbb{P}}_{2}+\left(\frac{d}{d t} p_{1}(t)\right) \hat{\mathbb{P}}_{1}\right]\left(e^{-n_{1}(t) \hat{H}_{4}}\right) e_{4}
\end{aligned}
$$

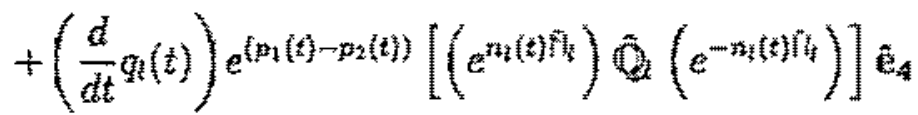

$$
\begin{aligned}
& +\left(\frac{a}{d t} n_{t}(t)\right) \hat{N}_{i} \hat{e}_{4}
\end{aligned}
$$

Da transformação de semelhança 3.5 podemos escrever:

$$
\left(e^{n_{2}(t) \bar{n}_{1}}\right)\left(\frac{d}{d t} p_{2}(t)\right) \hat{\mathbb{P}}_{2}\left(e^{-n_{1}(t) \hat{H}_{3}}\right)=\left(\frac{d}{d t} p_{2}(t)\right) \hat{\mathbb{P}}_{2}-\left(\frac{d}{d t} p_{2}(t)\right) n_{i}(t) \hat{N}_{1}
$$

80 


$$
\begin{aligned}
& \left(e^{n_{l}(t) n_{1}}\right)\left(\frac{d}{d t} p_{1}(t)\right) \hat{\mathbb{P}}_{1}\left(e^{-n t(t) \hat{t}_{1}}\right)=\left(\frac{d}{d t} p_{1}(t)\right) \hat{\mathbb{P}}_{1}+\left(\frac{d}{d t} p_{1}(t)\right) n_{l}(t) \hat{N}_{t} \\
& \left(e^{n_{t}(t) t_{i}}\right)\left(\frac{d}{d t} q_{l}(t)\right) e^{\left(p_{1}(t)-p_{2}(t)\right)} \hat{Q_{2}}\left(e^{-n_{i}(t) H_{4}}\right)= \\
& =\left(\frac{d}{d t} q_{l}(t)\right) e^{\left(p_{t}(t)-p_{2}(t)\right)}\left(\hat{Q}_{i}+n_{t}(t) \hat{P}_{2}-n_{l}(t) \hat{P}_{1}-n_{t}(t)^{2} \hat{\mathbb{N}_{l}}\right)
\end{aligned}
$$

e atijir nosso objetivo escrevendo:

$$
\begin{aligned}
& \left(\frac{d}{d t} \overrightarrow{\mathrm{e}}_{4}\right)=\hat{\mathbb{M}}_{1}\left[\left(\frac{d}{d t} m_{1}(t)\right)-n(t)\left(\frac{d}{d t} q(t)\right) e^{\left[m_{1}(t)-m_{2}(t)\right]}\right] \hat{\mathbf{e}}_{4} \\
& +\hat{B}_{2}\left[\left(\frac{d}{d t} m n_{2}(t)\right)+n(t)\left(\frac{d}{d t} q(t)\right) e^{\left(m,(t)-m_{2}(t)\right)}\right] \hat{\mathrm{e}}_{4} \\
& +\hat{Q}\left[\left(\frac{d}{d t} q(t)\right) e^{\left(m_{1}(t)-m_{n}(t)\right.}\right] \hat{\mathrm{e}}_{\boldsymbol{4}} \\
& +\hat{\mathrm{N}}\left[\left(\frac{d}{d t} n(t)\right)-n(t)^{2}\left(\frac{d}{d t} q(t)\right) e^{\left(m_{1}(t)-m_{2}(t)\right)}\right] \hat{\mathrm{e}}_{4} \\
& +\hat{N}\left[n(t)\left(\frac{d}{d t} m_{1}(t)\right)-n(t)\left(\frac{d}{d t} m_{2}(t)\right)\right] \hat{e}_{4} \\
& +\tilde{\mathbb{P}}_{\mathrm{I}}\left[\left(\frac{d}{d t} p_{1}(t)\right)-n_{l}(t)\left(\frac{d}{d t} q(t)\right) e^{\left(p_{1}(t)-p_{2}(t)\right)}\right] \hat{\mathbf{e}}_{4} \\
& +\hat{p}_{2}\left[\left(\frac{d}{d t} p_{2}(t)\right)+n_{t}(t)\left(\frac{d}{d t} q_{l}(t)\right) e^{\left(p_{1}(t)-p_{2}(t)\right)}\right] e_{e_{t}} \\
& +\hat{Q}_{i}\left[\left(\frac{d}{d t} q(t)\right) e^{\left\{p_{1}(t)-p_{2}(t)\right\}}\right] \hat{\mathrm{e}}_{4} \\
& +N\left[\left(\frac{d}{d t} n_{l}(t)\right)-n_{l}(t)^{2}\left(\frac{d}{d t} q(t)\right) e^{\left(p_{1}(t)-p_{2}(t)\right)}\right] \hat{t}_{4} \\
& +\hat{\mathrm{N}}_{1}\left[n_{1}(t)\left(\frac{d}{d t} p_{1}(t)\right)-n_{t}(t)\left(\frac{d}{d t} p_{2}(t)\right)\right] \hat{\mathrm{e}}_{4}
\end{aligned}
$$

5. A derivada no tempo da quínta definição $\hat{e}_{5} \equiv e^{z(i) \hat{\mathrm{e}}_{4}}$ é dada por:

$$
\begin{aligned}
& \left(\frac{d}{d t} \hat{e}_{3}\right)=\left(e^{z(t)}\right)\left(\frac{d}{d t} \hat{e}_{4}\right)+\left(\frac{d}{d t} z(t)\right) \hat{\mathbb{Z}} \hat{e}_{5} \\
& +\left(e^{z(t) \hat{t})} \hat{\mathbf{M}}_{1}\left[\left(\frac{d}{d t} m_{1}(t)\right)-n(t)\left(\frac{d}{d t} g(t)\right) e^{\left(m_{1}(t)-m_{2}(t)\right)}\right] \vec{e}_{3}\right.
\end{aligned}
$$




$$
\begin{aligned}
& +\left(e^{*(t) \hat{E}}\right) \mathrm{M}_{2}\left[\left(\frac{d}{d t} m_{2}(t)\right)+n(t)\left(\frac{d}{d t} q(t)\right) e^{\left(m_{1}(t)-m_{2}(t)\right.}\right] \hat{\mathrm{e}}_{4} \\
& +\left(e^{z(t)}\right) \hat{Q}\left[\left(\frac{d}{d t} q(t)\right) e^{\left(m_{1}(t)-m_{2}(t)\right)}\right] \hat{e}_{4} \\
& +\left(e^{z(t) \omega}\right) \mathrm{N}\left[\left(\frac{d}{d t} n(t)\right)-n(t)^{2}\left(\frac{d}{d t} q(t)\right) e^{\left(m_{3}(t)-m_{2}(t)\right)}\right] \hat{\mathrm{e}}_{4} \\
& +\left(e^{n t(t)}\right) \hat{N}\left[n(t)\left(\frac{d}{d t} m_{1}(t)\right)-n(t)\left(\frac{d}{d t} m_{2}(t)\right)\right] \hat{e}_{4}
\end{aligned}
$$

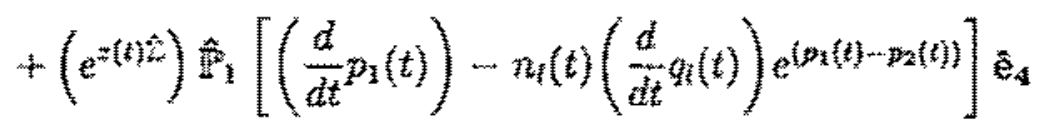

$$
\begin{aligned}
& +\left(e^{*(t)}\right) \hat{P}_{2}\left[\left(\frac{d}{d t} p_{2}(t)\right)+n_{1}(t)\left(\frac{d}{d t} q(t)\right) e^{(p t(t)-t+(t))}\right] \hat{e}_{4} \\
& +\left(e^{z(t)}\right) \mathbb{Q}_{t}\left[\left(\frac{d}{d t} g_{l}(t)\right) e^{\left(p_{1}(n)-p_{2}(t)\right)}\right] \hat{\mathrm{e}}_{4} \\
& +\left(p^{*(t) \hat{z}}\right) \hat{\mathbb{N}}_{l}\left[\left(\frac{d}{d t} n_{l}(t)\right)-n_{l}(t)^{2}\left(\frac{d}{d t} q_{l}(t)\right) e^{\left(p_{1}(t)-p_{2}(t)\right)}\right] \hat{\mathbf{e}}_{4} \\
& +\left(e^{z(t)}\right) N_{i}\left[n_{i}(t)\left(\frac{d}{d t} p_{1}(t)\right)-n_{t}(t)\left(\frac{d}{d t} p_{2}(t)\right)\right] \hat{e}_{4} \\
& +\left(\frac{d}{d t} z(t)\right) \hat{z}
\end{aligned}
$$

Utilizando os artificios ja mostrados e as relaçoes de comutaçă dadas em B.1, taremos:

$$
\begin{aligned}
& \left(\frac{d}{d t} \hat{e}_{s}\right)=\vec{M}_{Y}\left[\left(\frac{d}{d t} m_{1}(t)\right)-n(t)\left(\frac{d}{d t} q(t)\right) e^{\left.t m_{1}(n)-m_{2}(t)\right\rangle}\right] \hat{e}_{5} \\
& \left.+\left[\left(e^{z(t)}\right) \hat{M}_{2}\left(e^{-z(t) \hat{\omega}}\right)\right]\left[\frac{d}{d t} m_{2}(t)\right)+n(t)\left(\frac{d}{d t} q(t)\right) e^{\left(m_{1}(t)-m_{2}(t)\right)}\right] \hat{\mathbf{e}}_{5} \\
& +0\left[\left(\frac{d}{d t} q(t)\right) e^{\left(m_{1}(t)-m_{2}(t)\right)}\right] \hat{\mathrm{e}}_{5}
\end{aligned}
$$

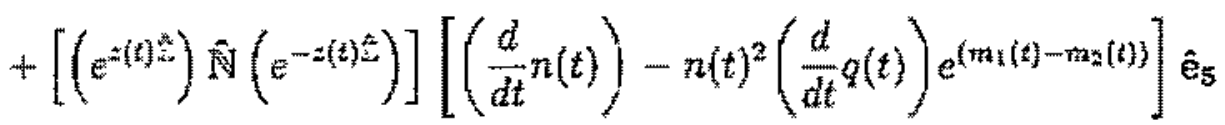

$$
\begin{aligned}
& +\left[\left(e^{2(t)}\right) \mathrm{A}\left(e^{-2(t) \ldots}\right)\right]\left[n(t)\left(\frac{d}{d t} m_{1}(t)\right)-n_{(t)}\left(\frac{d}{d t} m_{2}(t)\right)\right] \hat{e}_{3}
\end{aligned}
$$




$$
\begin{aligned}
& +\left[\left(e^{*(t) \hat{A})} p_{1}\left(e^{-z(t) \tilde{z}}\right)\right]\left[\left(\frac{d}{d t} p_{1}(t)\right)-m_{t}(t)\left(\frac{d}{d t} g_{t}(t)\right) e^{\left(p_{1}(t)-p_{2}(t)\right.}\right] \hat{e}_{5}\right. \\
& +\hat{\mathbb{P}}_{2}\left[\left(\frac{d}{d t} p_{2}(t)\right)+n_{l}(t)\left(\frac{d}{d t} q_{l}(t)\right) e^{\left(p_{1}(t)-p_{2}(t)\right)}\right] \hat{\mathbf{e}}_{5} \\
& +\left[\left(e^{z(t) \hat{2}}\right) \hat{C}_{d}\left(e^{-z(t) \hat{z}}\right)\right]\left[\left(\frac{d}{d t} q_{l}(t)\right) e^{\left(p_{1}(t)-p_{2}(t)\right)}\right] \hat{\mathrm{e}}_{5} \\
& +\tilde{N}_{i}\left[\left(\frac{d}{d t} n_{t}(t)\right)-n_{t}(t)^{2}\left(\frac{d}{d t} q_{t}(t)\right) e^{\left(p_{t}(t)-p_{2}(t)\right]}\right] \hat{\mathrm{e}}_{5} \\
& +\hat{N}_{t}\left[n_{l}(t)\left(\frac{d}{d t} p_{1}(t)\right)-n_{l}(t)\left(\frac{d}{d t} p_{2}(t)\right)\right] \hat{e}_{5} \\
& +\left(\frac{d}{d t} z(t)\right) \hat{\mathbb{Z}} \hat{\mathrm{e}}_{\mathrm{z}}
\end{aligned}
$$

Da transformaçäo de semelhança 3.5 podenos escrever:

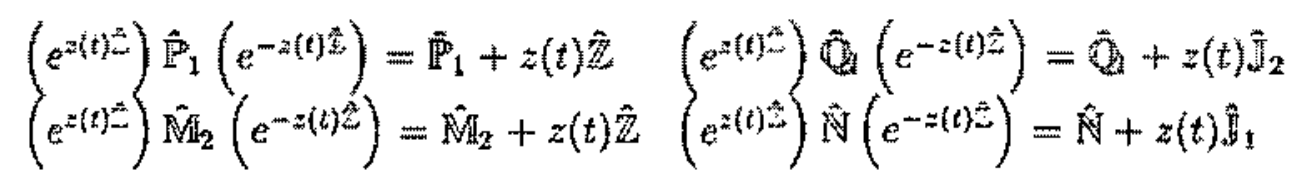

e atijir nosso objetivo escrevendo:

$$
\begin{aligned}
& \left(\frac{d}{d t} \hat{\mathbf{e}}_{5}\right)=\hat{\mathbf{M}}_{1}\left[\left(\frac{d}{d t} m_{1}(t)\right)-n(t)\left(\frac{d}{d t} q(t)\right) e^{\left(m_{2}(t)-m_{2}(t)\right)}\right] \hat{\mathbf{e}}_{5} \\
& +\hat{\mathrm{M}}_{2}\left[\left(\frac{d}{d t} m_{2}(t)\right)+n(t)\left(\frac{d}{d t} q(t)\right) e^{\left(m_{1}(t) \cdots m_{2}(t)\right)}\right] \hat{\mathrm{e}}_{\mathrm{S}} \\
& +\hat{\mathrm{Q}}\left[\left(\frac{d}{d t} q(t)\right) e^{\left(m_{2}(t)-m_{2}(t)\right)}\right] \hat{\mathrm{e}}_{5} \\
& +\hat{\mathrm{N}}\left[\left(\frac{d}{d t} \eta(t)\right)-n(t)^{2}\left(\frac{d}{d t} q(t)\right) e^{\left(m_{1}(t)-m_{2}(t)\right)}\right] \hat{\mathrm{e}}_{5} \\
& +\hat{\mathbb{N}}\left[n(t)\left(\frac{d}{d t} m_{1}(t)\right)-n(t)\left(\frac{d}{d t} m_{2}(t)\right)\right] \hat{\mathrm{e}}_{5} \\
& +\hat{\mathbb{E}}_{1}\left[\left(\frac{d}{d t} p_{1}(t)\right)-n_{I}(t)\left(\frac{d}{d t} q(t)\right) e^{\left(p_{1}(t)-p_{2}(t)\right)}\right] \hat{\mathrm{e}}_{5} \\
& +\hat{\mathbb{P}}_{2}\left[\left(\frac{d}{d t} p_{n}(t)\right)+n_{t}(t)\left(\frac{d}{d t} q(t)\right) e^{\left(p_{1}(t)-p_{2}(t)\right]}\right] \hat{\mathrm{e}}_{5} \\
& +\hat{Q}_{i}\left[\left(\frac{d}{d t} q(t)\right) e^{\left(p_{1}(t)-p_{2}(t)\right.}\right] \hat{\mathrm{e}}_{5}
\end{aligned}
$$




$$
\begin{aligned}
& +N_{i}\left[\left(\frac{d}{d t} n(t)\right)-n_{t}(t)^{2}\left(\frac{d}{d t} n(t)\right) e^{\left(p_{1}(t)-p_{m}(t)\right)} e_{5}\right. \\
& +N_{1}\left[n_{1}(t)\left(\frac{d}{d t} p_{1}(t)\right)-n_{t}(t)\left(\frac{d}{d t} p_{2}(t)\right)\right] \hat{e}_{s}
\end{aligned}
$$

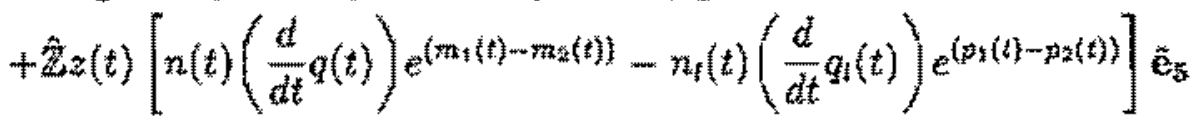

$$
\begin{aligned}
& +2\left[\left(\frac{d}{d t} z(t)\right)+z(t)\left(\left(\frac{d}{d t} p_{1}(t)\right)+\left(\frac{d}{d t} m_{2}(t)\right)\right)\right] \hat{\mathbf{e}}_{5} \\
& +\hat{H}_{1} z(t)\left[\left(\frac{d}{d t} n(t)\right)-n(t)^{2}\left(\frac{d}{d t} g(t)\right) e^{\left(m_{1}(t)-m_{z}(t)\right)}\right] \hat{\mathrm{e}}_{5} \\
& +\hat{H}_{1} z(t) n(t)\left[\left(\frac{d}{d t} m_{1}(t)\right)-\left(\frac{d}{d t} m_{2}(t)\right)\right] \hat{e}_{\mathrm{s}} \\
& +\hat{J}_{2}\left[z(t)\left(\frac{d}{d t} q(t)\right) e^{\left(p_{1}(t) \cdots p_{2}(t)\right)}\right] \hat{e}_{5}
\end{aligned}
$$

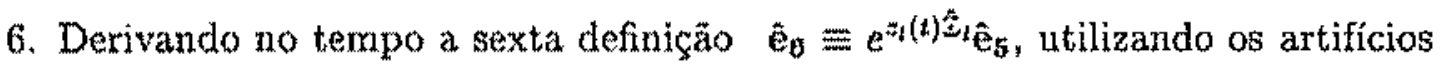
jẩ mostrados e as relaçós de comutaçäo dadas na tabela B.1 obtemos:

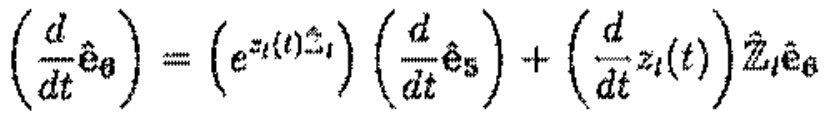

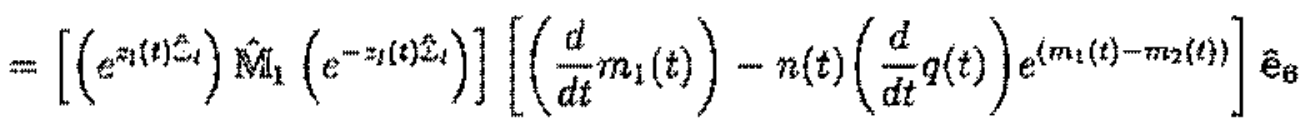

$$
\begin{aligned}
& +\hat{m}_{2}\left[\left(\frac{d}{d t} m_{2}(t)\right)+n(t)\left(\frac{d}{d t} q(t)\right) e^{\left(m_{1}(t)-m_{2}(t)\right)}\right] \hat{e}_{\sigma}
\end{aligned}
$$

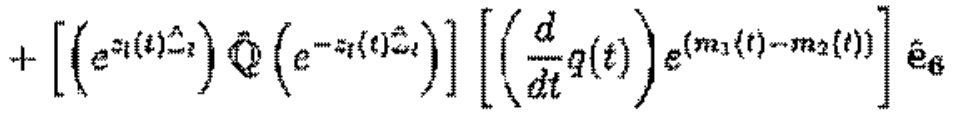

$$
\begin{aligned}
& +\hat{N}\left[\left(\frac{d}{d t} n(t)\right)-n(t)^{2}\left(\frac{d}{d t} q(t)\right) e^{\left(m_{2}(t)-m_{4}(t)\right)}\right] \hat{\mathbf{e}}_{\emptyset} \\
& +\hat{\mathrm{N}}\left[n(t)\left(\frac{d}{d t} m_{1}(t)\right)-m_{(t)}\left(\frac{d}{d t} m_{2}(t)\right)\right] e_{6}
\end{aligned}
$$

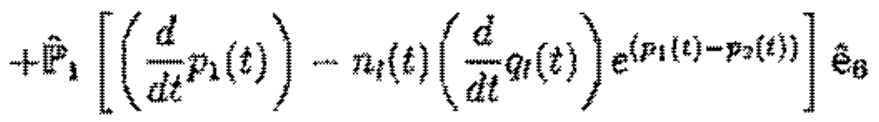

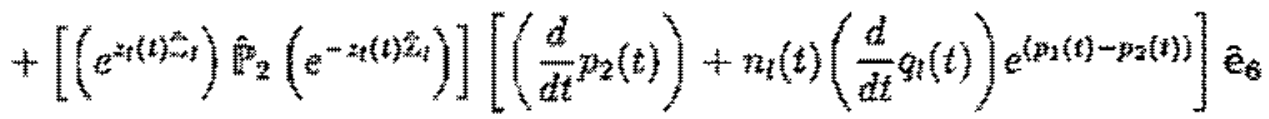




$$
\begin{aligned}
& +\mathbb{Q}\left[\left(\frac{d}{d t} q(t)\right) e^{\left(p_{1}\{t)-p_{2}(t)\right.}\right] \hat{\mathrm{e}}_{\mathrm{B}} \\
& +\left[\left(e^{z_{l}(t) \hat{\vec{s}}_{1}}\right) \hat{N}_{i}\left(e^{-z_{l}(t) \hat{\mathcal{L}}_{l}}\right)\right]\left[\left(\frac{d}{d t} n_{l}(t)\right)-n_{l}(t)^{2}\left(\frac{d}{d t} q_{l}(t)\right) e^{\left(p_{1}(t)-p_{2}(t)\right)}\right] \hat{\mathbf{e}}_{\boldsymbol{B}}
\end{aligned}
$$

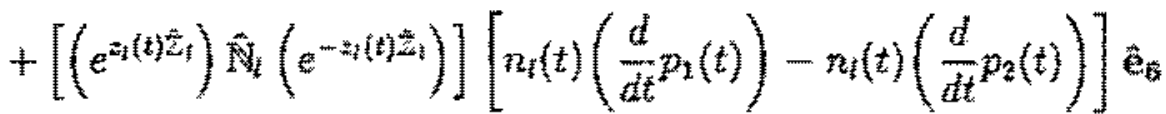

$$
\begin{aligned}
& +\hat{\mathbb{Z} z} z(t)\left[n(t)\left(\frac{d}{d t} q(t)\right) e^{\left(m_{1}(t)-m_{3}(t)\right)}-n_{i}(t)\left(\frac{d}{d t} q_{t}(t)\right) e^{\left(p_{1}(t)-\beta_{2}(t)\right)}\right] \hat{e}_{3} \\
& +\hat{\mathbb{Z}}\left[\left(\frac{d}{d t} z(t)\right)+z(t)\left(\left(\frac{d}{d t} p_{1}(t)\right)+\left(\frac{d}{d t} m_{2}(t)\right)\right)\right] \hat{\mathbf{e}}_{\boldsymbol{B}} \\
& +\hat{J}_{1} z(t)\left[\left(\frac{d}{d t} n(t)\right)-n(t)^{2}\left(\frac{d}{d t} q(t)\right) e^{\left(m_{1}(t)-n n_{2}(t)\right]}\right] \hat{\mathrm{e}}_{8} \\
& +\hat{\mathbb{J}}_{1} z(t) m(t)\left[\left(\frac{d}{d t} m_{1}(t)\right)-\left(\frac{d}{d t} m_{2}(t)\right)\right] \hat{e}_{6} \\
& +\hat{\mathbb{J}}_{2}\left[z(t)\left(\frac{d}{d t} q_{1}(t)\right) e^{\left(p_{1}(t)-p_{2}(t)\right\}}\right] \mathrm{e}_{6} \\
& +\left(\frac{d}{d t} z_{t}(t)\right) z_{i} \hat{e}_{\mathrm{e}}
\end{aligned}
$$

Da transformaçẫo de semelhança 3.5 podemos escrever:

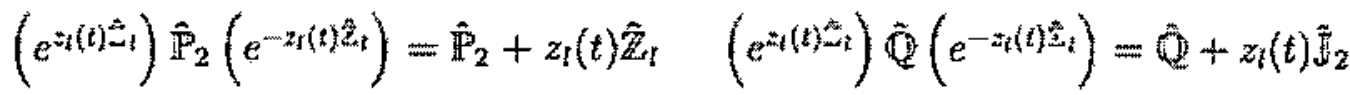

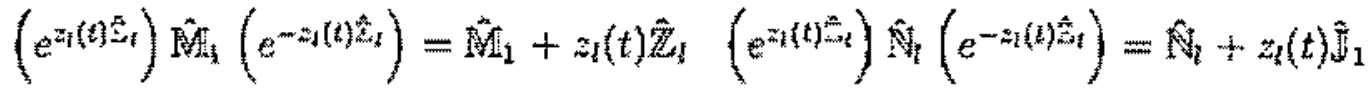

e atijir nosso objetivo escrevendo:

$$
\begin{aligned}
& \left(\frac{d}{d t} \hat{\mathrm{e}}_{6}\right)=\hat{\mathrm{M}}_{1}\left[\left(\frac{d}{d t} m_{1}(t)\right)-n(t)\left(\frac{d}{d t} q(t)\right) e^{\left(m_{1}(t)-m_{2}(t)\right)}\right] \hat{\mathrm{e}}_{3} \\
& +\hat{\mathrm{M}}_{2}\left[\left(\frac{d}{d t} m_{2}(t)\right)+n(t)\left(\frac{d}{d t} q(t)\right) e^{\left(m_{1}(t)-m_{2}(t)\right)}\right] \hat{\mathrm{e}}_{6} \\
& +\hat{\mathrm{Q}}\left[\left(\frac{d}{d t} q(t)\right) e^{\left(m_{2}(t)-m_{2}(t)\right)}\right] \hat{\mathrm{e}}_{\theta} \\
& +\hat{\mathbb{N}}\left[\left(\frac{d}{d t} n(t)\right)-n(t){ }^{2}\left(\frac{d}{d t} q(t)\right) e^{\left(m_{1}(t)-m_{2}(t)\right)}\right] \hat{\mathrm{e}}_{6}
\end{aligned}
$$




$$
\begin{aligned}
& +\hat{\mathbb{N}}\left[n(t)\left(\frac{d}{d t} m_{1}(t)\right)-n(t)\left(\frac{d}{d t} m_{2}(t)\right)\right] \hat{\mathbf{e}}_{6} \\
& +\hat{\mathbb{P}}_{1}\left[\left(\frac{d}{d t} p_{1}(t)\right)-n_{l}(t)\left(\frac{d}{d t} q_{l}(t)\right) e^{\left(p_{1}(t)-p_{2}(t)\right)}\right] \hat{\mathrm{e}}_{6} \\
& +\hat{\mathbb{P}}_{2}\left[\left(\frac{d}{d t} p_{2}(t)\right)+n_{l}(t)\left(\frac{d}{d t} q_{l}(t)\right) e^{\left(p_{1}(t)-p_{2}(t)\right\}}\right] \hat{\mathbf{e}}_{6} \\
& +\hat{\mathbb{Q}}_{i}\left[\left(\frac{d}{d t} q_{t}(t)\right) e^{\left(p_{1}(t)-p_{2}(t)\right)}\right] \hat{\mathbf{e}}_{\mathbf{B}} \\
& +\hat{\mathbb{N}}_{l}\left[\left(\frac{d}{d t} n_{l}(t)\right)-n_{l}(t)^{2}\left(\frac{d}{d t} q_{l}(t)\right) e^{\left(p_{2}(t)-p_{2}(t)\right)}\right] \hat{\mathbf{e}}_{6} \\
& +\hat{\mathbb{N}}_{l}\left[n_{l}(t)\left(\frac{d}{d t} p_{1}(t)\right)-n_{l}(t)\left(\frac{d}{d t} p_{2}(t)\right)\right] \hat{\mathbf{e}}_{8} \\
& +\hat{\mathbb{J}}_{1} z(t)\left[\left(\frac{d}{d t} n(t)\right)-n(t)^{2}\left(\frac{d}{d t} q(t)\right) e^{\left(m_{1}(t)-m_{2}(t)\right)}\right] \hat{\mathbf{e}}_{8} \\
& +\hat{\mathbb{J}}_{1} z(t) n(t)\left[\left(\frac{d}{d t} m_{1}(t)\right)-\left(\frac{d}{d t} m_{2}(t)\right)\right] \hat{\mathbf{e}}_{6} \\
& +\hat{\mathbb{J}}_{1} z_{l}(t)\left[\left(\frac{d}{d t} n_{l}(t)\right)-n_{l}(t)^{2}\left(\frac{d}{d t} q_{l}(t)\right) e^{\left(p_{1}(t)-p_{2}(t)\right)}\right] \hat{\mathbf{e}}_{6} \\
& +\hat{\mathbb{J}}_{1} z_{l}(t)\left[n_{l}(t)\left(\left(\frac{d}{d t} p_{1}(t)\right)-\left(\frac{d}{d t} p_{2}(t)\right)\right)\right] \hat{\mathbf{e}}_{6} \\
& +\hat{\mathbb{J}}_{2}\left[z(t)\left(\frac{d}{d t} q_{l}(t)\right) e^{\left(p_{1}(t)-p_{2}(t)\right)}\right] \hat{\mathrm{e}}_{6} \\
& +\hat{\mathbb{J}}_{2} z_{l}(t)\left[\left(\frac{d}{d t} m_{1}(t)\right)-n(t)\left(\frac{d}{d t} q(t)\right) e^{\left(m_{1}(t)-m_{2}(t)\right)}\right] \hat{\mathrm{e}}_{8} \\
& +\hat{\mathbb{Z}} z(t)\left[n(t)\left(\frac{d}{d t} q(t)\right) e^{\left(m_{2}(t)-m_{2}(t)\right)}-n_{l}(t)\left(\frac{d}{d t} g_{l}(t)\right) e^{\left(p_{1}(t)-p_{2}(t)\right)}\right] \hat{\mathbf{e}}_{6} \\
& +\hat{\mathbb{Z}}\left[\left(\frac{d}{d t} z(t)\right)+z(t)\left(\left(\frac{d}{d t} p_{1}(t)\right)+\left(\frac{d}{d t} m_{2}(t)\right)\right)\right] \hat{\mathbf{e}}_{6} \\
& +\hat{\mathbb{Z}}_{l}\left[\left(\frac{d}{d t} z_{l}(t)\right)+\left(\frac{d}{d t} m_{1}(t)\right)+\left(\frac{d}{d t} p_{2}(t)\right)\right] \hat{\mathrm{e}}_{\boldsymbol{\theta}} \\
& +\hat{\mathbb{Z}}_{l}\left[n_{l}(t)\left(\frac{d}{d t} q_{l}(t)\right) e^{\left(p_{1}(t)-p_{2}(t)\right\}}-n(t)\left(\frac{d}{d t} q(t)\right) e^{\left(m_{1}(t)-m_{2}(t)\right)}\right] \hat{\mathbf{e}}_{\mathbf{B}} .
\end{aligned}
$$




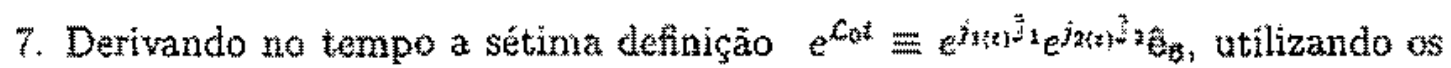
artifficios mencionados e as relaçotes de comutacão dedas pela tabela B.I obtemos:

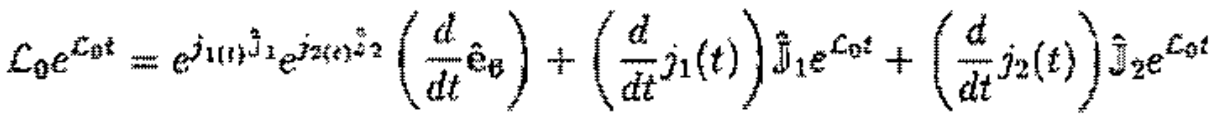

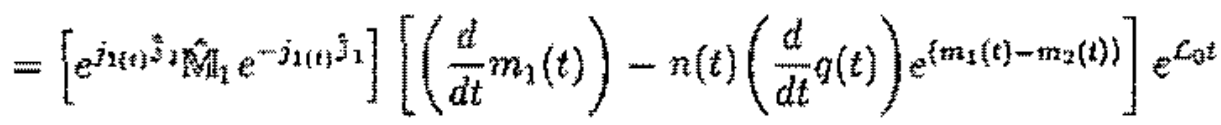

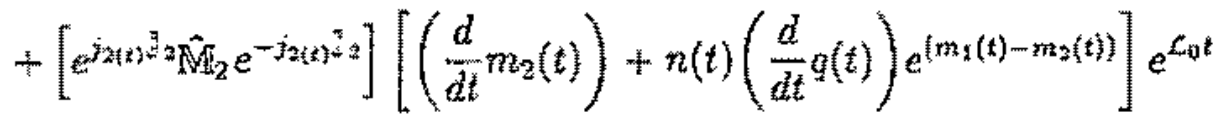

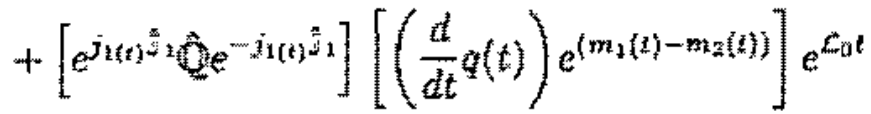

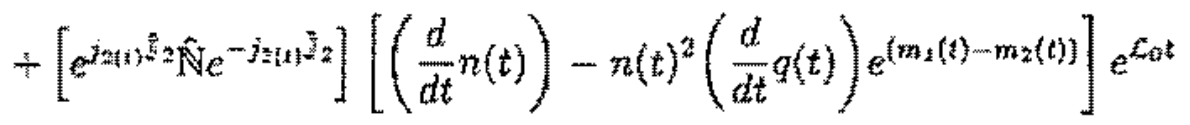

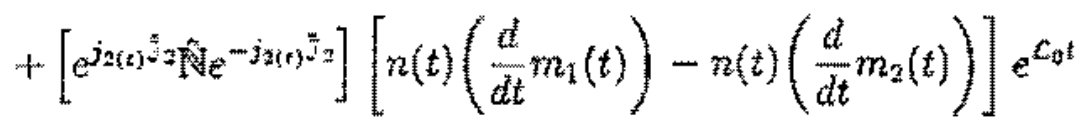

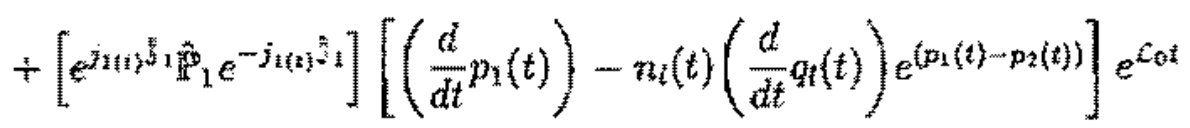

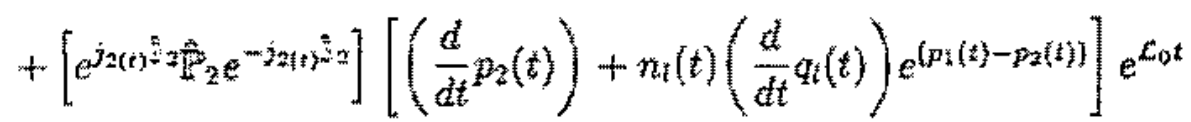

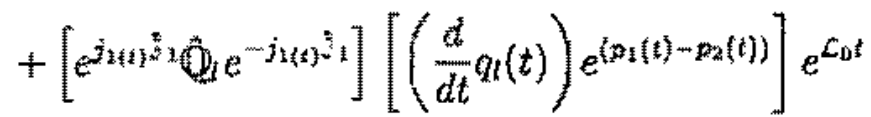

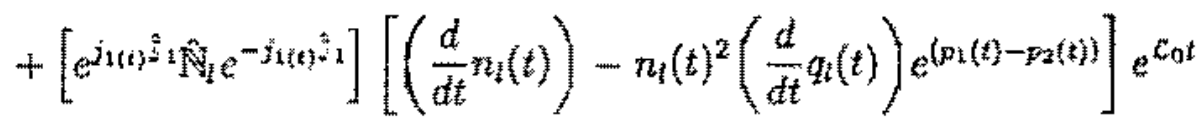

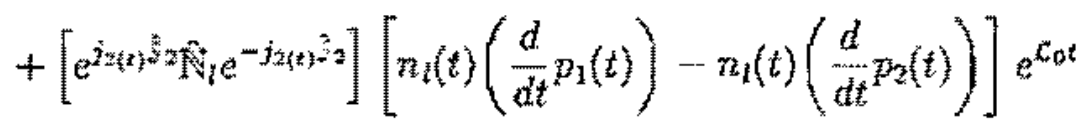

$$
\begin{aligned}
& +d_{1} z(t)\left[\left(\frac{d}{d t} n(t)\right)-n(t)^{2}\left(\frac{d}{d t} q(t)\right) e^{\left(m_{2}(t)-m_{2}(t)\right]}\right] e^{\mathcal{L}_{0} t} \\
& +\hat{J}_{1} z(t) n(t)\left[\left(\frac{d}{d t} m_{1}(t)\right)-\left(\frac{d}{d t} m_{2}(t)\right)\right] \mathrm{e}^{c_{0} t} \\
& +\hat{\mathbb{N}}_{1} z_{l}(t)\left[\left(\frac{d}{d t} n_{l}(t)\right)-n_{l}(t)^{2}\left(\frac{d}{d t} q(t)\right) e^{\left(p_{1}(t)-p_{2}(t)\right)}\right] e^{c_{0} t} \\
& +\hat{d}_{1} z_{l}(t)\left[n_{i}(t)\left(\left(\frac{d}{d t} p_{1}(t)\right)-\left(\frac{d}{d t} p_{2}(t)\right)\right)\right] e^{\varepsilon_{0} t}
\end{aligned}
$$




$$
\begin{aligned}
& +\left(\frac{d}{d t} j_{1}(t)\right) j_{1} e^{L_{0} t}+\left(\frac{d}{d t} j_{2}(t)\right) \hat{\mathbb{J}}_{2} e^{\mathcal{L}_{0} t} \\
& +H_{2}\left[z(t)\left(\frac{d}{d t} q_{l}(t)\right) e^{\left[p_{1}(t)-p_{2}(t)\right)}\right] e^{\mathcal{L}_{0} t} \\
& +H_{2} z_{1}(t)\left[\left(\frac{d}{d t} m_{1}(t)\right)-n(t)\left(\frac{d}{d t} a(t)\right) e^{\left(m_{t}(t)-m_{2}(t)\right)}\right] e^{t_{0 t} t}
\end{aligned}
$$

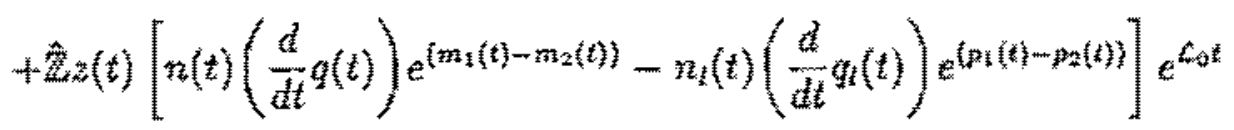

$$
\begin{aligned}
& +2\left[\left(\frac{d}{d t} z(t)\right)+z(t)\left(\left(\frac{d}{d t} p_{1}(t)\right)+\left(\frac{d}{d t} m_{2}(t)\right)\right)\right] e^{t_{t} t} \\
& +H_{k}\left[\left(\frac{d}{d t} z_{1}(t)\right)+\left(\frac{d}{d t} m_{1}(t)\right)+\left(\frac{d t}{d t} p_{2}(t)\right)\right] e^{C_{1} t} \\
& +\tilde{B}_{l}\left[n_{l}(t)\left(\frac{d}{d t} q_{l}(t)\right) e^{\left(p_{1}(t)-p_{2}(t)\right)}-n(t)\left(\frac{d}{d t} q(t)\right) e^{\left(m_{1}(t)-m_{2}(t)\right)}\right] e^{c_{0} t} \text {. }
\end{aligned}
$$

Da transformação de semelhança 3.5 podemos escrever:

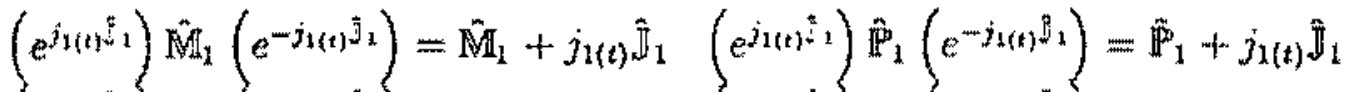

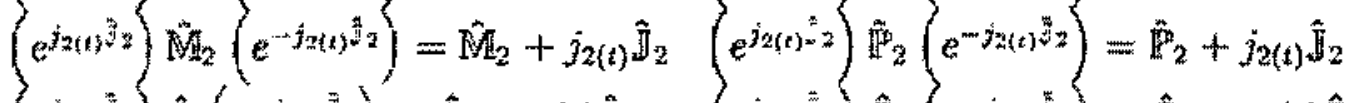

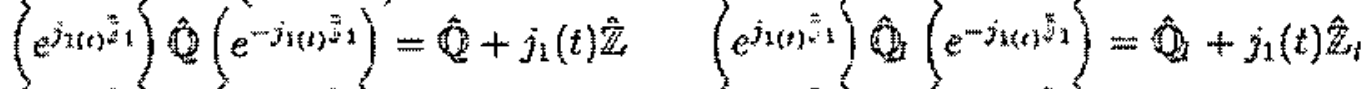

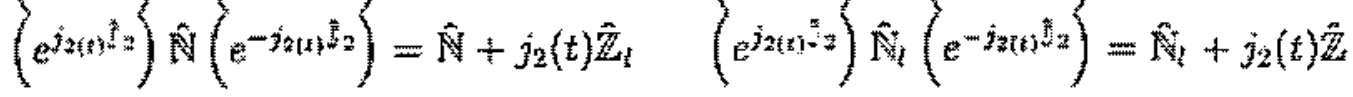

e atiut nosso objetivo final escrevendo:

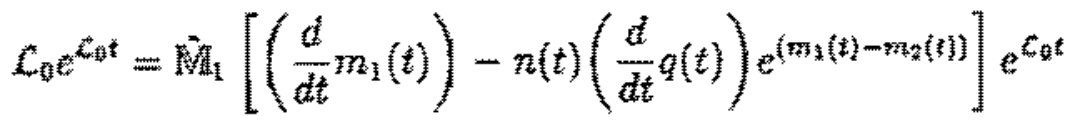

$$
\begin{aligned}
& +M_{2}\left[\left(\frac{d}{d t} m_{2}(t)\right)+n(t)\left(\frac{d}{d t} q(t)\right) e^{t m_{1}(t)-m_{2}(t)}\right] e^{f_{0} t}
\end{aligned}
$$

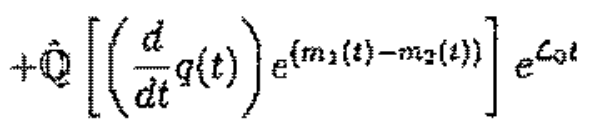

$$
\begin{aligned}
& +N\left[\left(\frac{d}{d t} n(t)\right)-n(t)^{2}\left(\frac{d}{d t} q(t)\right) e^{\left(m_{t}(t)-n_{3}(n)\right]}\right] e^{f_{0} t} \\
& +\left[n(t)\left(\frac{d}{d t} m_{1}(t)\right)-n(t)\left(\frac{d}{d t} m_{2}(t)\right)\right] e^{c_{0} t} \\
& +\hat{\mathbb{P}}_{1}\left[\left(\frac{d}{d t} p_{1}(t)\right)-n_{1}(t)\left(\frac{d}{d t} q_{1}(t)\right) e^{\left(p_{1}(t)-p_{n}(t)\right)}\right] e^{c_{1} t}
\end{aligned}
$$




$$
\begin{aligned}
& +E_{2}\left[\left(\frac{d}{d t} p_{2}(t)\right)+n_{i}(t)\left(\frac{d}{d t} q_{t}(t)\right) e^{\left(p_{1}(t)-p_{3}(t)\right)}\right] e^{t_{0} t} \\
& +Q_{t}\left[\left(\frac{d}{d t} a_{1}(t)\right) e^{\left(p_{1}(t)-p_{3}(t)\right)}\right] e^{\cos _{0} t}
\end{aligned}
$$

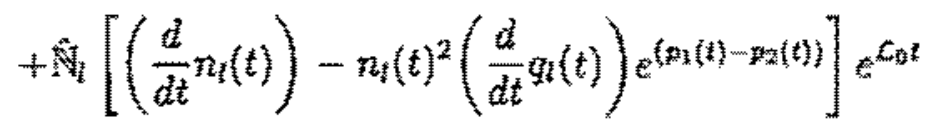

$$
\begin{aligned}
& +\mathbb{N}_{1}\left[n_{1}(t)\left(\frac{d}{d t} p_{1}(t)\right)-n_{1}(t)\left(\frac{d}{d t} p_{2}(t)\right)\right] e^{\cos t} \\
& +\hat{n} z(t)\left[\left(\frac{d}{d t} n(t)\right)-n(t)^{2}\left(\frac{d}{d t} q(t)\right) e^{\left(m_{1}(t)-m_{n}(t)\right]}\right] e^{c_{a t}} \\
& +\tilde{J}_{1} z(t) n(t)\left[\left(\frac{d}{d t} m_{1}(t)\right)-\left(\frac{d}{d t} m_{2}(t)\right)\right] e^{t_{0} t} \\
& \left.+\tilde{\mathbb{N}}_{1} z_{l}(t)\left[\left(\frac{d}{d t} n_{l}(t)\right)-n_{l}(t)^{2}\left(\frac{d}{d t} q_{l}(t)\right)\right] e^{\left(p_{i}(t)-p_{n}(t)\right)}\right] e^{\tilde{L}_{0} t} \\
& +\hat{\mathbb{I}}_{1} z_{l}(t)\left[n_{l}(t)\left(\left(\frac{d}{d t} p_{1}(t)\right)-\left(\frac{d}{d t} p_{2}(t)\right)\right)\right] e^{\hat{x}_{0} t} \\
& +\hat{H}_{1} \dot{j}_{1}(t)\left[\left(\frac{d}{d t} m_{1}(t)\right)-n(t)\left(\frac{d}{d t} q(t)\right) e^{\left(m_{1}(t)-m_{2}(t)\right)}\right] e^{L_{0} t} \\
& +\hat{H}_{1} j_{1}(t)\left[\left(\frac{d}{d t} p_{1}(t)\right)-n_{t}(t)\left(\frac{d}{d t} q(t)\right) e^{\left(p_{1}(t)-p_{2}(t)\right)}\right] e^{c_{n} t} \\
& +\vec{N}\left(\frac{d}{d t} j_{1}(t)\right) e^{c_{u t}} \\
& +\tilde{H}_{2}\left(\frac{d}{d t} \hat{f}_{2}(t)\right) \mathrm{e}^{\mathcal{E}_{0} t}
\end{aligned}
$$

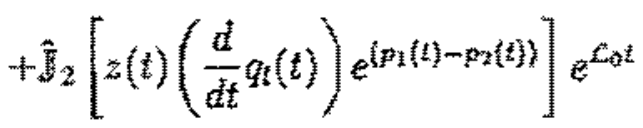

$$
\begin{aligned}
& +\tilde{\mathbb{J}}_{2} z_{i}(t)\left[\left(\frac{t}{d t} m_{1}(t)\right)-n(t)\left(\frac{d}{d t} \phi(t)\right) e^{\left(m_{1}(t)-m_{3}(t)\right)}\right] e^{c_{0} t} \\
& \div J_{2} j_{2}(t)\left[\left(\frac{d}{d t} m_{2}(t)\right)+n(t)\left(\frac{d}{d t} g(t)\right) e^{\left(m m_{1}(t)-m_{2}(t)\right)}\right] e^{c_{0} t t} \\
& +\hat{J}_{2} j_{2}(t)\left[\left(\frac{d}{d t} p_{2}(t)\right)+n_{l}(t)\left(\frac{d}{d t} q_{r}(t)\right) e^{\left(p_{2}(t)-p_{2}(t)\right)}\right] e^{c_{0} t}
\end{aligned}
$$




$$
\begin{aligned}
& +\hat{Z}_{z}(t)\left[n(t)\left(\frac{d}{d t} q(t)\right) e^{\left(m_{1}(t)-m_{2}(t)\right)}-n_{l}(t)\left(\frac{d}{d t} q(t)\right) e^{\left(p_{1}(t) \cdots p_{2}(t)\right)}\right] e^{L_{0} t} \\
& +\hat{\mathbb{Z}}\left[\left(\frac{d}{d t} z(t)\right)+z(t)\left(\left(\frac{d}{d t} p_{1}(t)\right)+\left(\frac{d}{d t} m_{2}(t)\right)\right)\right] e^{\mathcal{L}_{0} t} \\
& +\hat{Z}\left[j_{1}(t)\left(\frac{d}{d t} q(t)\right) e^{\left(m_{1}(t)-m_{2}(t)\right)}+j_{2}(t)\right] e^{L_{0} t}
\end{aligned}
$$

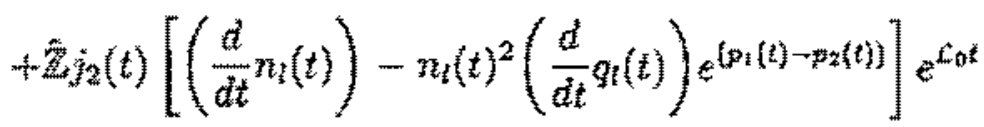

$$
\begin{aligned}
& +\hat{z}_{j}(t) \eta_{t}(t)\left[\left(\frac{d}{d t} p_{1}(t)\right)-\left(\frac{d}{d t} p_{2}(t)\right)\right] e^{c_{0} t} \\
& +\hat{z}_{i}\left[\left(\frac{d}{d t} z_{t}(t)\right)+\left(\frac{d}{d t} m_{1}(t)\right)+\left(\frac{d}{d t} p_{2}(t)\right)\right] e^{t_{0} t} \\
& +\hat{\mathbb{Z}}_{i}\left[r_{t}(t)\left(\frac{d}{d t} q(t)\right) e^{\left(p_{1}(t)-p_{2}(t)\right)}-n(t)\left(\frac{d}{d t} q(t)\right) e^{\left(m_{1}(t)-m_{3}(t)\right)}\right] e^{c_{j} t} \\
& +\hat{Z}_{i} \hat{j}_{2}(t)\left[\left(\frac{d}{d t} n(t)\right)-n(t)^{2}\left(\frac{d}{d t} q(t)\right) e^{\left(m_{2}(t)-m_{3}(t)\right)}\right] e^{c_{0} t} \\
& +\dot{Z}_{1} j_{2}(t) n(t)\left[\left(\frac{d}{d t} m_{1}(t)\right)-\left(\frac{d}{d t} m_{2}(t)\right)\right] e^{c_{0} t}
\end{aligned}
$$

Escrevendo a equaçắo para $\mathcal{L}_{0}$ em termos dos superoperadores $\hat{a ̂ y}_{9} \hat{a}_{2}$ obtemos:

$$
\begin{aligned}
& L_{0}=\hat{\mathbf{a}}_{\mathbf{1}}^{\dagger} \hat{a}_{1} \cdot\left[\left(\frac{d}{d t} m_{1}(t)\right)-n(t)\left(\frac{d}{d t} d(t)\right) e^{\left(m_{1}(n)-m_{2}(t)\right.}\right] \\
& +\hat{a}_{2}^{\hat{z}_{2}} \cdot\left[\left(\frac{d}{d t} m_{2}(t)\right)+n(t)\left(\frac{d}{d t} q(t)\right) e^{\left(m_{2}(t)-m_{2}(t)\right)}\right] \\
& +a_{2}^{t} \hat{a}_{2} \cdot\left[\left(\frac{d}{d t} q(t)\right) e^{\left(m_{1}(t)-m_{2} t(t)\right.}\right] \\
& +\hat{a}_{1} \hat{a}_{2} \cdot\left[\left(\frac{d}{d t} n(t)\right)-n(t)^{2}\left(\frac{d}{d t} q(t)\right) e^{\left\{m_{1}(t)-m_{2}(t)\right)}\right] \\
& \text { +tầ } \hat{a}_{2}^{t} \cdot\left[n(t)\left(\frac{d}{d t} m_{1}(t)\right)-n(t)\left(\frac{d}{d t} m_{2}(t)\right)\right] \\
& +\cdot \hat{\mathbf{a}}_{1}^{\dagger} \hat{\mathbf{a}}_{1}\left[\left(\frac{d}{d t} p_{1}(t)\right)-n_{l}(t)\left(\frac{d}{d t} q_{l}(t)\right) e^{\left(p_{1}(t)-p_{2}(t)\right)}\right] \\
& +\hat{a}_{2}^{t} \hat{a}_{2}\left[\left(\frac{d}{d t} p_{2}(t)\right)+n_{l}(t)\left(\frac{d}{d t} g_{l}(t)\right) e^{\left(p_{1}(t)-p_{2}(t)\right)}\right]
\end{aligned}
$$




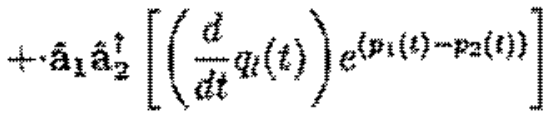

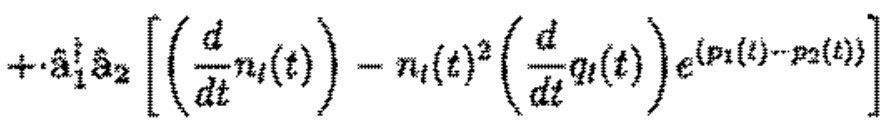

$$
\begin{aligned}
& +x_{1}^{*} a_{2}\left[n_{1}(t)\left(\frac{d}{d t} p_{1}(t)\right)-n_{1}(t)\left(\frac{d}{d t} p_{2}(t)\right)\right] \\
& +\hat{a}_{1} \cdot a_{1}^{t} z(t)\left[\left(\frac{d}{d t} n(t)\right)-n(t)^{2}\left(\frac{d}{d t} q(t)\right) e^{\left(m n_{1}(t) m_{2} n_{2}(t)\right)}\right] \\
& +\hat{a}_{1} \cdot a_{1} z_{(t) n(t)}\left[\left(\frac{d}{d t} m_{1}(t)\right)-\left(\frac{d}{d t} m_{2}(t)\right)\right] \\
& \left.+\hat{\mathbf{a}}_{1} \cdot \mathbf{a}_{1}^{t} z_{t}(t)\right]\left[\left(\frac{d}{d t} n_{1}(t)\right)-n_{1}(t)^{2}\left(\frac{d}{d t} q_{1}(t)\right) e^{\left(p_{1}(t)-p_{2}(t)\right]}\right] \\
& +a_{3} \cdot a_{1}^{i} z_{l}(t)\left[n_{1}(t)\left(\left(\frac{d}{d t} p_{1}(t)\right)-\left(\frac{d}{d t} p_{2}(t)\right)\right)\right] \\
& +\hat{a}_{1} \cdot \hat{a}_{1}^{\dagger} j_{1}(t)\left[\left(\frac{d}{d t} m_{1}(t)\right)-n(t)\left(\frac{d}{d t} q(t)\right) e^{\left[m_{1}(t)-m_{2}(t)\right)}\right] \\
& +\hat{\mathbf{a}}_{1} \cdot \hat{\mathbf{a}}_{1}^{\dagger} j_{1}(t)\left[\left(\frac{d}{d t} p_{1}(t)\right)-n_{l}(t)\left(\frac{d}{d t} q_{l}(t)\right) e^{\left(p_{1}(t)-p_{2}(t)\right)}\right] \\
& +\overrightarrow{\mathbf{a}}_{1} \cdot \hat{\mathbf{a}}_{\mathbf{1}}\left(\frac{d}{d t} j_{1}(t)\right) \\
& +\hat{a}_{2} \cdot \hat{a}_{2}^{\dagger}\left(\frac{d}{d t} j_{2}(t)\right) \\
& +\hat{\mathbf{a}}_{2} \cdot \hat{\mathbf{a}}_{2}^{t}\left[z(t)\left(\frac{d}{d t} q(t)\right) e^{\left(p_{1}(t)-p_{2}(t)\right)}\right] \\
& +\vec{a}_{2} \cdot \vec{a}_{z}^{t} \tilde{z}_{l}(t)\left[\left(\frac{d}{d t} m_{1}(t)\right)-n(t)\left(\frac{d}{d t} q(t)\right) e^{\left\{m_{1}(t)-m_{3}(t)\right]}\right] \\
& +\hat{a}_{2} \cdot \hat{a}_{2}^{t} j_{2}(t)\left[\left(\frac{d}{d t} m_{2}(t)\right)+n(t)\left(\frac{d}{d t} q(t)\right) e^{\left(m_{1}(t) m m_{2}(t)\right)}\right] \\
& +\hat{a}_{2}+\hat{a}_{2}^{\dagger} j_{2}(t)\left[\left(\frac{d}{d t} p_{2}(t)\right)+r_{t}(t)\left(\frac{d}{d t} q_{(}(t)\right) e^{\left.(p)(t)-p_{z}(t)\right)}\right] \\
& +a_{2} \cdot a_{1}^{y} z(t)\left[n(t)\left(\frac{d}{d t} q(t)\right) e^{\left(m_{3}(t)-m_{2}(t)\right)}-n_{1}(t)\left(\frac{d}{d t} q_{t}(t)\right) e^{\left(m_{t}(t)-p_{3}(t)\right)}\right]
\end{aligned}
$$




$$
\begin{aligned}
& +\hat{\mathbf{a}}_{2} \cdot \hat{\mathbf{a}}_{1}^{\dagger}\left[\left(\frac{d}{d t} z(t)\right)+z(t)\left(\left(\frac{d}{d t} p_{1}(t)\right)+\left(\frac{d}{d t} m_{2}(t)\right)\right)\right] \\
& +\vec{a}_{2} \cdot \vec{a}_{1}^{t}\left[j_{1}(t)\left(\frac{d}{d t} q(t)\right) e^{\left(m_{1}(t)-m_{2}(t)\right)}+j_{2}(t)\right] \\
& +a_{2}+a_{1}^{t} j_{2}(t)\left[\left(\frac{d}{d t} n_{1}(t)\right)-m_{t}(t)^{2}\left(\frac{d}{d t} q_{n}(t)\right) e^{\left\{p_{1}(t)-p_{2}(t)\right)}\right] \\
& +\mathrm{a}_{2}+\mathrm{u}_{1} \mathrm{~g}_{2}(t) n_{1}(t)\left[\left(\frac{d}{d t} p_{1}(t)\right)-\left(\frac{d}{d t} p_{2}(t)\right)\right]
\end{aligned}
$$

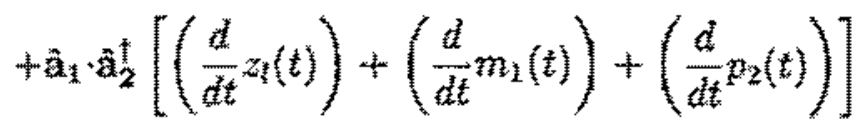

$$
\begin{aligned}
& +a_{1} \cdot a_{2}\left[n_{1}(t)\left(\frac{d}{d t} q(t)\right) e^{\left.(p)(t)-p_{2}(t)\right)}-n(t)\left(\frac{d}{d t} \sigma_{(t)}\right) e^{\left(m_{1}(t)-m_{2}(t)\right)}\right] \\
& +a_{1} \cdot a_{2} j_{2}(t)\left[\left(\frac{d}{d t} n(t)\right)-n(t)^{2}\left(\frac{d}{d t} q(t)\right) e^{\left.i m_{1}(t)-m_{2}(t)\right)}\right] \\
& +\hat{\mathbf{a}}_{1} \cdot \hat{\mathrm{a}}_{2}^{\dagger} j_{2}(t) n(t)\left[\left(\frac{d}{d t} m_{1}(t)\right)-\left(\frac{d}{d t} m_{2}(t)\right)\right] \text {. }
\end{aligned}
$$

\section{B.3 Cálculo dos coeficientes}

Se compararmos os coeficientes da derivada obtida acima com o liouvilliano, $\mathcal{L}_{0}$, da seçâă 4.1, equaçäo 4.15, encontraremos um sistema de equaçöes lineares que apresentaremos a seguir.

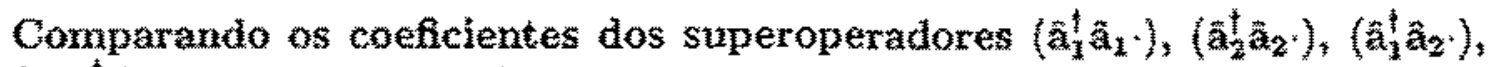
( $\left.\hat{a}_{1} \hat{a}_{2}^{t}\right)$, obtemos respectivamente:
1) $\left(\frac{d}{d t} m_{1}(t)\right)-n(t)\left(\frac{d}{d t} q(t)\right) e^{\left(m_{1}(t)-m_{2}(t)\right)}=-n_{2}-k_{1}$
2) $\left(\frac{d}{d t} m_{2}(b)\right) * n(t)\left(\frac{d}{d t} q(t)\right) e^{\left\{m_{1}(t)-m_{2}(t)\right\}}=-i \Omega_{2}-h_{2}$
3) $\left(\frac{d}{d t} q(t)\right) e^{\left(m_{1}(t)-m_{n}(t)\right)}=-k_{3}$ 
4) $\left(\frac{d}{d t} n(t)\right)+n(t)\left[\left(\frac{d}{d t} m_{1}(t)\right)-\left(\frac{d}{d t} m_{2}(t)\right)\right]-n(t)^{2}\left(\frac{d}{d t} q(t)\right) e^{\left(m_{1}(t)-m_{z}(t)\right)}=-k_{3}$

Comparando os coeficientes os superoperadores $\left(\cdot \hat{a}_{1}^{\dagger} \hat{a}_{1}\right),\left(\cdot \hat{a}_{2}^{\dagger} \hat{a}_{2}\right),\left(\cdot \hat{a}_{1} \hat{a}_{2}^{\dagger}\right)$, ( $\hat{\mathrm{a}}_{1}^{\dagger} \hat{\mathrm{a}}_{2}$ ), obtemos respectivamente:

5) $\left(\frac{d}{d t} p_{1}(t)\right)-n_{1}(t)\left(\frac{d}{d t} q_{l}(t)\right) e^{\left(p_{1}(t)-p_{2}(t)\right)}=i \Omega_{1}-k_{1}$

6) $\left(\frac{d}{d t} p_{2}(t)\right)+n_{t}(t)\left(\frac{d}{d t} q(t)\right) e^{\left(p_{1}(t)-p_{2}(t)\right)}=i \Omega_{2}-k_{2}$

7) $\left(\frac{d}{d t} q_{l}(t)\right) e^{\left(p_{1}(t)-p_{2}(t)\right)}=-k_{3}$

8) $\left(\frac{d}{d t} n_{l}(t)\right)+n_{t}(t)\left[\left(\frac{d}{d t} p_{1}(t)\right)-\left(\frac{d}{d t} p_{2}(t)\right)\right]-n_{l}(t)^{2}\left(\frac{d}{d t} q_{l}(t)\right) e^{\left(p_{1}(t)-p_{3}(t)\right)}=-k_{3}$

Ao resolver o sistema de equaçōes $1,2,3,4$, que é simétrico ao sistema de equaçōes $5,6,7,8$, encontraremos uma equação de Riccati cuja soluçāo nos fornecerá os coeficientes que estamos procurando:

$$
\begin{aligned}
& q(t)=n(t)=(q(t))^{*}=\left(n_{l}(t)\right)^{*} \\
& q(t)=2 k_{3}\left(1-e^{+t}\right)\left(\Delta_{+} e^{r t}-\Delta_{-}\right)^{-1} \text { para } p
\end{aligned}
$$

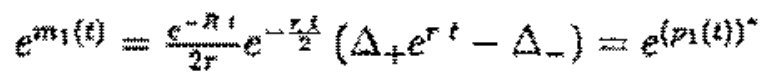

$$
\begin{aligned}
& e^{m_{2}(t)}=e^{-2 R t} e^{-m_{3}(t)}=e^{\left(p_{2}(t)\right)^{*}} \\
& r=\sqrt{c^{2}+4 k_{3}^{2}}, \quad c=k_{2}-k_{1}+i\left(\Omega_{2}-\Omega_{1}\right), \\
& R=\frac{k+k_{1}}{2}+\frac{i\left(\Omega_{2}+\Omega_{1}\right)}{2}, \quad \Delta_{(4)}=c\left(\begin{array}{l}
+ \\
m
\end{array}\right)
\end{aligned}
$$

Note que $m_{1}(t)$ e $m_{2}(t)$ tendem a $-\infty$ quando $t \rightarrow \infty$, visto que

$$
\left(h_{1}+h_{2}\right)>\operatorname{Re}\{r\}
$$


Com efeito, se $\left(k_{1}+k_{2}\right) \geq \Re e\{r\}$ entāo $\left(k_{1}+k_{2}\right)^{2} \geq(\Re e\{r\})^{2}$, pois $k_{1}$ e $k_{2}$ são positivos.

A equaçăo B.2 mostra que

$$
\begin{aligned}
\left(k_{1}+k_{2}\right)^{2} & >\left(\{e\{\tau\})^{2} \Longrightarrow\right. \\
\left(k_{1}+k_{2}\right)^{2} & >\frac{1}{2}\left\{\left(k_{1}-k_{1}\right)^{2}+4 k_{3}^{2}-\left(\Omega_{2}-\Omega_{1}\right)^{2}\right\} \\
& +\frac{1}{2} \sqrt{\left\{\left(k_{2}-k_{1}\right)^{2}+4 k_{3}^{2}-\left(\Omega_{2}-\Omega_{1}\right)^{2}\right\}^{2}+2\left(\left(\Omega_{2}-\Omega_{1}\right)\left(k_{2}-k_{1}\right)+4 k_{3}\right\}^{2}} \\
\left\{2\left(k_{1}+k_{2}\right)^{2}-\left\{\left(k_{2}-k_{1}\right)^{2}+4 k_{3}^{2}-\left(\Omega_{2}-\Omega_{1}\right)^{2}-4 g^{2}\right\}\right\}^{2}> & \\
\left\{\left(k_{2}-k_{1}\right)^{2}\right. & \left.+4 k_{3}^{2}-\left(\Omega_{2}-\Omega_{1}\right)^{2}-4 g^{2}\right\}^{2}+2\left(\left(\Omega_{2}-\Omega_{1}\right)\left(k_{2}-k_{1}\right)+4 g k_{3}\right\}^{2}
\end{aligned}
$$

Como $k_{3}^{2}=k_{1} k_{2}$ então a desigualdade torna-se,

$$
\left\{2 k_{3}\left(\Omega_{2}-\Omega_{1}\right)-2 g\left(k_{2}-k_{1}\right)\right\}^{2}>0
$$

que é sempre verdadeiro, comprovando assim a desigualdade da squaçäo B.3.

Note ainda que se $k_{1}=k_{2}=k_{3}=k$ e $\omega_{1}=\omega_{2}=\omega$ teremos

$$
\left.\begin{array}{l}
q(t)=\left(1-e^{2 k t}\right)\left(1+e^{2 k t}\right)^{-1} \\
1+q(t)=2\left(1+e^{2 k t}\right)^{-1} \\
1-q(t)=e^{2 k t}(1+q(t)) \\
e^{m_{1}(t)}=e^{-R t} e^{-k t}(1+q(t))^{-1} \\
e^{m_{2}(t)}=e^{m_{1}(t)}(1+q(t))(1-q(t))
\end{array}\right\}
$$

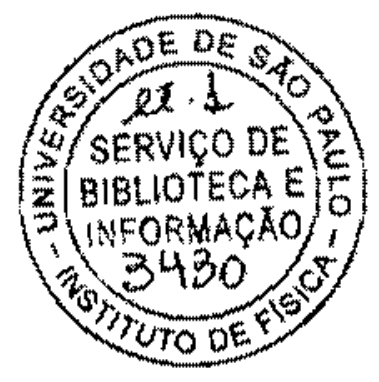




\section{Referências Bibliográficas}

[1] F, Dalfowo et al., Reu, Mod. Phys., 71, 463 (1999);

[2] J.P. Paz, W.H. Zurek, Phys. Rev. Lett. , 82, 26 (1999);

[3] K.M. Fonseca Romero, M.C.Nemes, Phys. Lett. A235, 432 (1997);

[4] V.J. Menon, N. Chamana, Y. Singh, Prog. Theor, Phys., 98, 321 (1097);

[5] J.I. Kim, M.C. Nemes, A.F.R de Toledo Piza, H.E.Borges, Phys. Rez. Leth, $77,207(1906)$

[6] M. Brune et al., Phys, Rev. Lett. , 77, 4887 (1996);

[7] L.Davidovich, M. Brune, J.M.Raimond,S. Haroche, Phys. Rev., A53, 1295 (1996);

[8] L. Mandel, E. Wolf, Optical Coherence and Quantum Optżs, (Cambridge University Press, New York, 1995);

[9] D.F. Walls e G.J. Milburn, Quantum Optics, (Springer, Berlin, 1995);

[10] W.H. Zurek, S. Habib, J.P.Paz, Phys. Rev. Lett. , 70, 1187 (1993);

[11] N. Gisin, I.C.Percival, Journal Physics, A25, 5677 (1992);

[12] B.L.Hu, Juan P.Paz, Yuhong Zhang, Phys. Rey., D45, 2843 (1992);

[13] W.H. Zurek, Phys. Todag, , 44, 36 (1991);

[14] O.N. Borges, Fatoraçăo da funçâo Exponencial do Liontillaño de Eqquaçóes Mestra Tese de doutorado UFMG Belo Horizonte 1991;

[15] L. de la Peña, Introdaccón a la mecánica cuántica, (Ediciones científicas uni versitárias, México, 1991);

[16] C.W. Gardiner, Quantum Noise, (Springer, Berlin, 1991);

[17] G.Ghirardi, A.Rimini, P.Pearde, in "Sixty-Two Years of Uncertaknty", A. Miller (ed) (Plenum, New York (1090); 
[18] M.C. Nemes, A.F.R de Toledo Piza, Physica A, 137,367 (1086);

[19] A.O. Caldeira, A.J. Leggett, Phys. Rev., A31, 1050 (1985);

[20] A.O. Caldeira, A.J. Letggett, Physica, A 121, 587 (1983);

[21] A.O. Caldeira, A.J. Leggett, Ann. Phys., 149, 374 (1983);

[22] J. Wheler, W.H. Zurek, Quantum Theory and Mesurement, (Princeton University Press 1983);

[23] W. Witschel, Intemational Journal of Quantum Ohemistry, 20, 1233m1241 (1981):

[24] JM. Normand, A Lie Group: Rotations in Quantum Mechanics Amsterdam: North-Holland, 1980

[25] A. Welrt, Rev. Mod. Phys, , 50, 221 (1978);

[26] C. Cohen-Tannoudji, B. Diu,F. Laloê, Quantum Mechanics, (Wiley, New York, 1977);

[27] M. Suzuki, Communtetions in Mathematical Physics, 57, 193-200 (1977);

[28] R. Gilmore, Lie Groups, Lie Algebras, and Some of Their Applications, (Wiley, New York, 1974);

[29] R. Gilmore, Journal of Mathematical Physics, 15, 2090-2092 (1974);

[30] W.H. Louisell, Quantum Statistical Properties of Radiation, (Wiley, New York, 1973);

[31] M.D. Kostin, Journal Chemistry Physics, 57, 3589 (1972);

[32] H. Fröhlich, International Joumal of Quantum Chemistry, v.II, 641-649 (1968);

[33] R.M. Wilcox, Journal of Mathematical Physics, 8, 962-982 (1967);

[34] K.Kumar, Journal of Mathematical Physics, 6, 1928-1934 (1965);

[35] J. von Neumann, Mathematical Found,ations of Qutantam Mechanics, (Princeton University Press 1955);

[36] W. Magnus, Commanications on Pure and Apptied Mathematics, VII, 649-673 $(1954) ;$

[37] E. Kanai, Prog. Theor. Phys., 3, 440 (1948);

[38] P. Caldirola, Nuovo Cimento, 18, 397 (1941);

[39] E.Schrödinger, Naturuissenschaffen, 23, 807,823 e 844 (1935); 